Pacific

Journal of

Mathematics

ON THE SUCCESSIVE APPROXIMATION OF CLASSICAL SOLUTIONS FOR MULTI-SURFACE FREE-BOUNDARY PROBLEMS WITH GENERAL NON-LINEAR JOINING CONDITIONS

ANDREW ACKer 


\title{
ON THE SUCCESSIVE APPROXIMATION OF CLASSICAL SOLUTIONS FOR MULTI-SURFACE FREE-BOUNDARY PROBLEMS WITH GENERAL NON-LINEAR JOINING CONDITIONS
}

\author{
ANDREW ACKER
}

\begin{abstract}
We prove the global convergence of an analytical trial freeboundary algorithm, called the operator method, in the context of a very large class of multiple-free-boundary problems in $\mathbb{R}^{N}, N \geq 2$. We study the general case of a finite number of annular flow-layers, having a nested family of closed, $(N-1)$-dimensional hypersurfaces as interfaces. Each interface is characterized by a general non-linear joining condition relating the normal derivatives of the stream functions in the two adjoining layers.
\end{abstract}

\section{Introduction and main results.}

The main purpose of this paper is to prove the global convergence of an analytical trial free-boundary method, called the operator method, for the successive approximation of classical solutions of general multi-layer freeboundary problems. Our results are in the context of the following problem with general non-linear joining conditions across the unknown nested layerinterfaces:

1.1. Problem. In $\mathbb{R}^{N}(N \geq 2)$, for given $\Re_{0} \geq 0$, let $\mathbb{X}\left(\Re_{0}\right)$ denote the family of all simple closed $(N-1)$-dimensional hypersurfaces $S$ in $\mathbb{R}^{N}$ such that $S \cup D(S)$ is starlike relative to $\left\{x \in \mathbb{R}^{N}:|x| \leq \Re_{0}\right\}$, where $D(S)$ (resp. $E(S)$ ) denotes the interior (exterior) complement of $S$. In $\mathbb{X}\left(\Re_{0}\right)$, we write $S_{1}<S_{2}$ (resp. $\left.S_{1} \leq S_{2}\right)$ iff $S \cup D\left(S_{1}\right)$ (resp. $D\left(S_{1}\right)$ ) is a subset of $D\left(S_{2}\right)$. For any $k \in \mathbb{N}$, let $\mathbf{X}_{k}\left(\Re_{0}\right)$ denote the family of all ordered $k$-tuples $\boldsymbol{S}=\left(S_{1}, \ldots, S_{k}\right) \in\left[\mathbb{X}\left(\Re_{0}\right)\right]^{k}$ such that $S_{1}<S_{2}<\cdots<S_{k}$. Given $k \in \mathbb{N}$, a pair $\left(S_{*}^{-}, S_{*}^{+}\right) \in \mathbf{X}_{2}\left(\Re_{0}\right)$ such that $S_{*}^{-}$(resp. $S_{*}^{+}$) has an interior (exterior) tangent ball at every point, and a family of $C^{1}$-functions $F_{i}(x, p, q): \mathbb{R}^{N} \times \mathbb{R}_{+} \times \mathbb{R}_{+} \rightarrow \mathbb{R}, i=1, \ldots, k$, we seek a $C^{1}$-multisurface $\boldsymbol{S}=\left(S_{1}, \ldots, S_{k}\right) \in \mathbf{X}_{k}\left(\Re_{0}\right)$ such that $S_{*}^{-}<S_{i}<S_{*}^{+}$for $i=1, \ldots, k$, and

$$
F_{i}\left(x,\left|\nabla U_{i}(x)\right|,\left|\nabla U_{i+1}(x)\right|\right)=0 \quad \text { on } S_{i}
$$

for $i=1, \ldots, k$. Here $U_{i}(x)$ solves the boundary-value problem

$$
\Delta U_{i}(x)=0 \quad \text { in } \Omega_{i}, \quad U_{i}\left(S_{i-1}\right)=0, \quad U_{i}\left(S_{i}\right)=1,
$$


where $S_{0}:=S_{*}^{-}, S_{k+1}:=S_{*}^{+}, \Omega_{i}:=D\left(S_{i}\right) \cap E\left(S_{i-1}\right)$, and $\Delta$ denotes the Laplace operator.

Our study of Problem 1.1 is restricted to the case where each given $C^{1}$ function $F:=F_{i}(x, p, q): \mathbb{R}^{N} \times \mathbb{R}_{+} \times \mathbb{R}_{+} \rightarrow \mathbb{R}$ has the following properties:

(A1) We have $\partial F / \partial p<0<\partial F / \partial q$ wherever $F$ is defined.

(A2) We have $F\left(x_{0}+\lambda v, p / \lambda, q / \lambda\right) \geq 0$ for any $x_{0}, v \in \mathbb{R}^{N}, p, q \in \mathbb{R}_{+}$, and $\lambda \in[1, \infty)$ such that $F\left(x_{0}+v, p, q\right) \geq 0$ and $\left|x_{0}\right| \leq \Re_{0}$.

(A3) For any $\eta>0$ and any compact set $K \subset \mathbb{R}^{N}$, there exists a value $\mu$ so large that $F(x, p, q)>0$ whenever $x \in K, p \in(0, \eta]$, and $q \geq \mu$, and $F(x, p, q)<0$ whenever $x \in K, q \in(0, \eta]$, and $p \geq \mu$.

The above assumptions are all satisfied if for each $i=1, \ldots, k$, the joining function is in the form $F_{i}(x, p, q)=q^{\beta_{i}}+A_{i}(x)-p^{\alpha_{i}}$, where $0<\beta_{i} \leq \alpha_{i}$, and where $A_{i}(x): \mathbb{R}^{N} \rightarrow \mathbb{R}_{+}$is a strictly-positive continuous function such that, for any $x_{0}, v \in \mathbb{R}^{N}$ with $\left|x_{0}\right| \leq \Re_{0}$, the related function $\phi_{i}(t):=$ $t^{\alpha_{i}} A_{i}\left(x_{0}+t v\right)$ is weakly increasing in $t \geq 0$. In the important special case where $\alpha_{i}=\beta_{i}=2$ for each $i$, Problem 1.1 can be interpreted as a flow of $k+1$ immiscible ideal fluids (with stream functions $U_{1}, \ldots, U_{k+1}$ ) such that the flow-speeds on the flow interfaces $S_{1}, \ldots, S_{k}$ satisfy Bernoulli's law in the form

$$
\left|\nabla U_{i}(x)\right|^{2}=\left|\nabla U_{i+1}(x)\right|^{2}+A_{i}(x) \text { on } S_{i}
$$

for $i=1, \ldots, k$. Flow problems with joining conditions in the form (1.3) have been extensively studied in arbitrary dimensions (see $[4,5, \mathbf{1 1}, \mathbf{1 6}]$ ). The author showed in [4, Thm. 3.1] that Problem 1.1 has a unique multisurface solution $\boldsymbol{S} \in \mathbf{X}_{k}\left(\Re_{0}\right)$ provided that each joining condition (1.1) is in the form (1.3), where $A_{i}(x)$ is a strictly-positive $C^{\infty}$-function such that $\phi(t):=t^{2} A_{i}\left(x_{0}+t v\right)$ is weakly increasing in $t \geq 0$ for any $x_{0}, v \in \mathbb{R}^{N}$ with $\left|x_{0}\right| \leq \Re_{0}$. The author showed in [7] that if $\boldsymbol{S}=\left(S_{1}, \ldots, S_{k}\right)$ is any $k$-tuple of nested, simple closed $(N-1)$-dimensional $C^{2}$-hypersurfaces such that (1.1) and (1.2) hold, then $\boldsymbol{S}$ is uniquely determined and actually $\boldsymbol{S} \in \mathbf{X}_{k}\left(\Re_{0}\right)$, provided that $\left(S_{0}, S_{k+1}\right) \in \mathbf{X}_{2}\left(\Re_{0}\right)$ and the joining functions $F_{i}$ all satisfy (A1) and (A2). In [8], the author obtained convex existence results for Problem 1.1 under suitable convex conditions.

1.2. Operator definition. Assume in Problem 1.1 that the functions $F_{i}(x, p, q), i=1, \ldots, k$, satisfy Assumptions (A1)-(A3). Let

$$
\begin{aligned}
\mathbb{Y} & =\mathbb{Y}\left(\Re_{0}, S_{*}^{ \pm}\right) \\
& :=\left\{\boldsymbol{S}=\left(S_{1}, \ldots, S_{k}\right) \in \mathbf{X}_{k}\left(\Re_{0}\right): S_{*}^{-}<S_{i}<S_{*}^{+} \text {for } i=1, \ldots, k\right\} .
\end{aligned}
$$

Our successive approximation algorithm is based on the mappings

$$
\boldsymbol{T}_{\varepsilon}(\boldsymbol{S})=\boldsymbol{T}(\boldsymbol{S} ; \varepsilon): \mathbb{Y} \rightarrow \mathbb{Y}, \varepsilon \in(0,1) .
$$


Here, the $i$-th component of $\boldsymbol{T}_{\varepsilon}(\boldsymbol{S}) \in \mathbb{Y}$ is given by

$$
T_{\varepsilon, i}(\boldsymbol{S})=\left\{x \in \omega_{\varepsilon, i}: F_{i}\left(x,\left(\varepsilon / d\left(x, S_{\varepsilon, i}^{-}\right)\right),\left(\varepsilon / d\left(x, S_{\varepsilon, i}^{+}\right)\right)\right)=0\right\},
$$

for any $\boldsymbol{S}=\left(S_{1}, \ldots, S_{k}\right) \in \mathbb{Y}$, where we define $\omega_{\varepsilon, i}:=E\left(S_{\varepsilon, i}^{-}\right) \cap D\left(S_{\varepsilon, i}^{+}\right)$, $S_{\varepsilon, i}^{+}:=\left\{U_{i+1}(x)=\varepsilon\right\}$, and $S_{\varepsilon, i}^{-}:=\left\{U_{i}(x)=1-\varepsilon\right\}$. Here, the functions $U_{i}$ solve (1.2) relative to $\boldsymbol{S}=\left(S_{1}, \ldots, S_{k}\right)$, and $d(\cdot, \cdot)$ denotes the Euclidian distance between two sets (or a point and a set).

1.3. Fixed point problem. For any $\varepsilon \in(0,1)$, we seek $\widetilde{\boldsymbol{S}}_{\varepsilon} \in \mathbb{Y}$ such that $\boldsymbol{T}_{\varepsilon}\left(\widetilde{\boldsymbol{S}}_{\varepsilon}\right)=\widetilde{\boldsymbol{S}}_{\varepsilon}$.

Definition 1.4. We define the metric $\boldsymbol{M}$ in $\mathbf{x}_{k}(0)$ such that

$$
\boldsymbol{M}\left(\boldsymbol{S}_{1}, \boldsymbol{S}_{2}\right):=\inf \left\{\lambda \geq 0: \exp (-\lambda) \boldsymbol{S}_{1} \leq \boldsymbol{S}_{2} \leq \exp (\lambda) \boldsymbol{S}_{1}\right\}
$$

for any $\boldsymbol{S}_{1}=\left(S_{1,1}, \ldots, S_{1, k}\right), \boldsymbol{S}_{2}=\left(S_{2,1}, \ldots, S_{2, k}\right) \in \mathbf{X}_{k}(0)$, where, for $\alpha>0$, we define the multisurface $\alpha \boldsymbol{S}_{1} \in \mathbf{X}_{k}(0)$ and the multi-surface inequalities $\boldsymbol{S}_{1} \leq \boldsymbol{S}_{2}, \boldsymbol{S}_{1}<\boldsymbol{S}_{2}$ component-wise $\left(\alpha \boldsymbol{S}_{1}=\left(\alpha S_{1,1}, \ldots, \alpha S_{1, k}\right)\right.$, where $\alpha \Sigma:=\{\alpha x: x \in \Sigma\}$ for any set $\Sigma \subset \mathbb{R}^{N}$, and $\boldsymbol{S}_{1} \leq \boldsymbol{S}_{2}$ (resp. $\left.\boldsymbol{S}_{1}<\boldsymbol{S}_{2}\right) \Leftrightarrow S_{1, i} \leq S_{2, i}\left(\right.$ resp. $\left.S_{1, i}<S_{2, i}\right)$ for $\left.i=1, \ldots, k\right)$.

Our purpose is to study the convergence properties of the operator method of successive approximations, in which, for any given initial multisurface $\boldsymbol{S}_{0} \in \mathbb{Y}$ and any given sequence $\left(\varepsilon_{n}\right)_{n=0}^{\infty}$ of values in the interval $(0,1)$, the sequence $\left(\boldsymbol{S}_{n}\right)_{n=0}^{\infty}$ of multisurfaces in $\mathbf{Y}$ is defined recursively such that

$$
\boldsymbol{S}_{n+1}=\boldsymbol{T}\left(\boldsymbol{S}_{n} ; \varepsilon_{n}\right)
$$

for $n=0,1,2, \ldots$ For the case where $\varepsilon_{n}=\varepsilon \in(0,1)$ and $\boldsymbol{S}_{n}=\boldsymbol{T}_{\varepsilon}^{n}\left(\boldsymbol{S}_{0}\right)$ for all $n$, we will prove (under Assumptions (A1)-(A3)) that for all initial multisurfaces $\boldsymbol{S}_{0} \in \mathbb{Y}$ having a specified degree of componentwise separation, we have

$$
\boldsymbol{M}\left(\boldsymbol{S}_{n}, \widetilde{\boldsymbol{S}}_{\varepsilon}\right) \leq C \alpha^{n} \boldsymbol{M}\left(\boldsymbol{T}_{\varepsilon}\left(\boldsymbol{S}_{0}\right), \boldsymbol{S}_{0}\right)
$$

for all $n \in \mathbb{N}$, where $C>0$ and $\alpha \in(0,1)$ are suitable constants and $\widetilde{\boldsymbol{S}}_{\varepsilon} \in \mathbb{Y}$ denotes the unique multi-surface solution of Problem 1.3 (see $§ 7.11$ ). This establishes a geometric convergence rate for the fixed- $\varepsilon$ iteration. Under the additional assumption that the solution $\widetilde{\boldsymbol{S}} \in \mathbb{Y}$ of Problem 1.1 exists as a $C^{1,1}$-multisurface, we prove (see $\S 9.2$ ) that

$$
\boldsymbol{M}\left(\widetilde{\boldsymbol{S}}_{\varepsilon}, \widetilde{\boldsymbol{S}}\right) \rightarrow 0 \quad \text { as } \varepsilon \downarrow 0 .
$$

We will also prove (see $\S 9.3$ ) that if the classical solution $\widetilde{\boldsymbol{S}} \in \mathbb{Y}$ of Problem 1.1 is a $C^{1,1}$-multisurface, then

$$
\boldsymbol{M}\left(\boldsymbol{S}_{n}, \widetilde{\boldsymbol{S}}\right) \rightarrow 0 \quad \text { as } n \rightarrow \infty
$$

provided that $\left(\varepsilon_{n}\right)_{n=0}^{\infty}$ is any null sequence such that $\sum_{n=0}^{\infty} \varepsilon_{n}=\infty$. 
The "operator method" is a trial free-boundary method, in the sense of determining $\boldsymbol{S}_{n+1}$ in terms of the capacitary potentials of the multisurface $\boldsymbol{S}_{n}$ for each $n \in \mathbb{N}$. For general discussions of trial free-boundary methods for one-free-boundary problems in elliptic PDE's, we refer the reader to Crank [12] and Cryer [13]. Like any trial free-boundary method, the operator method is highly geometric, in the sense that the iterates are all multisurfaces. To the author's knowledge, no other trial free-boundary method has been applied to Problem 1.1, and no other has yielded to a general mathematical convergence analysis.

This paper is devoted to proving (1.9), (1.10) and (1.11). Our main proofs, in $\S \S 7,9$, are assembled componentwise, according to organizing principles related to the construction of weighted metrics and of inner and outer solutions, from several uniform 2-surface, 3-surface, and 4-surface operator estimates developed for the purpose in $\S \S 3,5,6,8$. For the fixed point results in $\S 7$, we first construct families of multisurfaces in $\mathbb{Y}$, each of which is an inner or outer solution of Problem 1.3 at all $\varepsilon \in(0,1)$ simultaneously. This is accomplished by multiple application of an estimate for ordered triples of similar surfaces given in $§ 3.1$. Secondly, for any given $\boldsymbol{S} \in \mathbb{Y}$ and $\varepsilon \in(0,1)$, we show, by multiple application of a 4 -surface separation estimate given in $\S 5.1$, that the component-surfaces of the operator iterates $\boldsymbol{T}_{\varepsilon}^{n}(\boldsymbol{S})$ are pairwise separated by a distance which is independent of $n \in \mathbb{N}$. Thirdly, for any fixed $\varepsilon \in(0,1)$, we apply a 3 -surface operator estimate obtained in $\S 6.1$ componentwise to construct a weighted multisurface metric $\boldsymbol{M}_{\varepsilon}$ such that the operator $\boldsymbol{T}_{\varepsilon}$, applied to multisurfaces with sufficiently-separated components, is a contraction relative to $\boldsymbol{M}_{\varepsilon}$. Once the metric $\boldsymbol{M}_{\varepsilon}$ has been constructed (for any fixed $\varepsilon \in(0,1)$ ), the geometric convergence of the multi-surface operator iterates to the multi-surface fixed point $\widetilde{\boldsymbol{S}}_{\varepsilon}$ easily follows by the familiar contraction-mapping principle. The proof of (1.10) is a natural by-product of the proof of (1.11), which we discuss next. In the first place, the proof of (1.11) does not follow from the fixed- $\varepsilon$ convergence results of $\S 7$, due to the critical dependence of the multi-surface metric $\boldsymbol{M}_{\varepsilon}$ on $\varepsilon$. Our proof of (1.11) is based on the construction in $\S 9$, by multiple application of 3 -surface operator estimates in $\S 8.4$, of continuously and monotonically-varying parametrized families of inner and outer solutions of Problem 1.1, which we denote by $\boldsymbol{S}^{ \pm}(r), r \in\left[0, r_{0}\right]$. The construction is such that $\boldsymbol{S}^{ \pm}(0)=\widetilde{\boldsymbol{S}}, \boldsymbol{S}^{-}\left(r_{0}\right) \leq \boldsymbol{S} \leq \boldsymbol{S}^{+}\left(r_{0}\right)$ for all $\boldsymbol{S} \in \mathbb{Y}$, and, for some constant $C>0$ and null function $z(\cdot)$, we have

$$
\boldsymbol{T}_{\varepsilon}\left(\boldsymbol{S}^{-}(r)\right) \geq \boldsymbol{S}^{-}(\phi(r, \varepsilon)) ; \boldsymbol{T}_{\varepsilon}\left(\boldsymbol{S}^{+}(r)\right) \leq \boldsymbol{S}^{+}(\phi(r, \varepsilon))
$$

for all $r \in\left[0, r_{0}\right]$ and $\varepsilon \in(0,1)$, where $\phi(r, \varepsilon):=(1-C \varepsilon) r+\varepsilon z(\varepsilon)$. The second convergence theory is closely related to the first. The relationship is perhaps best exhibited by the fact that $\left\{\boldsymbol{S} \in \mathbb{Y}: \boldsymbol{S}^{-}(r) \leq \boldsymbol{S} \leq \boldsymbol{S}^{+}(r)\right\}=$ $\left\{\boldsymbol{S} \in \mathbb{Y}: \boldsymbol{M}_{0}(\boldsymbol{S}, \widetilde{\boldsymbol{S}}) \leq r\right\}$ for all $r \in\left(0, b_{0}\right]$, where the weighted multi-surface 
metric $\boldsymbol{M}_{0}$ and the constant $b_{0}>0$ are suitably chosen. Thus, the estimates (1.12) are actually globally-applicable generalizations of the estimate:

$$
\boldsymbol{M}_{0}\left(\boldsymbol{T}_{\varepsilon}(\boldsymbol{S}), \widetilde{\boldsymbol{S}}\right) \leq(1-C \varepsilon) \boldsymbol{M}_{0}(\boldsymbol{S}, \widetilde{\boldsymbol{S}})+\varepsilon z(\varepsilon),
$$

which holds for all $\varepsilon \in(0,1)$ and all $\boldsymbol{S} \in \mathbb{Y}$ such that $\boldsymbol{M}_{0}(\boldsymbol{S}, \widetilde{\boldsymbol{S}}) \leq b_{0}$. Essentially, the estimate (1.13) introduces a uniform metric by restricting attention to the distance of the operator iterates from the classical solution $\widetilde{\boldsymbol{S}}$. This idea is not applicable in the fixed-point approach.

The author has previously studied the convergence of successive approximations for the operator method for Problem 1.1 in the case of 2 layers and one free surface $(k=1$; see $[3],[4, \S 4],[6])$. Since the present multisurface operator definition consists of the componentwise application of the earlier definition, it is important to emphasize that the present convergence results do not follow by multiple (componentwise) application of the previous results or operator estimates. While the earlier papers treated operator iterations of one free surface between two known $C^{2}$-surfaces, nothing is known about the immediate neighbors of a particular free surface in the present context unless it follows from the analysis itself. For example, the unknown neighboring free surfaces have no known smoothness properties, because the operator has not been shown theoretically to preserve any particular degree of smoothness. Therefore, the present proof of (1.9) must be based on operator estimates applicable to practically arbitrary 3 -surface configurations. Uniform separation of components, which was not an issue in the one-free-surface case, is a key factor in this proof. While some one-sided smoothness is retained in the construction of the inner and outer solution families $\boldsymbol{S}^{ \pm}(r), r \in\left[0, r_{0}\right]$, used in the proof of (1.11), the inductive multi-surface constructions are necessarily very complex relative to the 2-layer case.

Remark 1.5. Recently, extensive numerical studies of the operator method have been carried out in the work of Kadakal [15] and Acker, Kadakal, and Miller [9]. Further study is in progress. These studies show that the operator method can be adapted to a variety of free boundary problems (including the one-layer fluid problem with or without geometric constraints on the free boundary, the two-layer fluid problem, and the Prandtl-Batchelor problem), where it often converges quite quickly and painlessly even in geometric situations not quite covered by the author's analytical convergence proofs. In this context, the purpose of the present paper is to provide the full theoretical justification for extending the same numerical methods to the multi-surface case.

Remark 1.6. The author's theoretical results for the operator method do not answer the question of the best choice of the parameter $\varepsilon_{n}$ at the 
$n$-th step of the iteration. Although it has not been shown that the operator iterates $\boldsymbol{S}_{n}$ remain smooth, no computational examples have been encountered in which they did not appear to do so. In view of this apparent smoothness, one encounters the following trade-off in the application of the operator $\boldsymbol{T}_{\varepsilon}$ : It is preferable to choose $\varepsilon \in(0,1)$ large, because (a) the forward progression of the multisurface induced by the operator application is approximately proportional to $\varepsilon$, and (b) the difficulty of computing the operator iteration increases as $\varepsilon$ decreases, because the grid dimension in the computation of the capacitary potentials must decrease correspondingly. On the other hand, it is preferable for $\varepsilon \in(0,1)$ to be small, because the fixed point $\widetilde{\boldsymbol{S}}_{\varepsilon}$ of $\boldsymbol{T}_{\varepsilon}$ is then a more accurate approximation of the classical solution $\widetilde{\boldsymbol{S}}$. Due to (a) and (b), it is important that the sequence $\left(\varepsilon_{n}\right)$ not approach zero too fast, since this would result in "apparent convergence" of the iterates short of reaching the actual solution. It is also important that $\left(\varepsilon_{n}\right)$ not approach zero too slowly, since the accuracy of the $n$-th iterate (as an approximation of the solution) will generally not be better than the accuracy of the "fixed point" $\widetilde{\boldsymbol{S}}_{\varepsilon}$ at $\varepsilon=\varepsilon_{n}$. The following has proved to be a safe, routine procedure (see $[\mathbf{9}, \mathbf{1 5}]$ ): First iterate at a fixed, "large" value of $\varepsilon$, such as $\varepsilon=1 / 2$, until the iterates are near the fixed point $\widetilde{\boldsymbol{S}}_{1 / 2}$, then continue the iteration at a new, smaller value, such as $\varepsilon=1 / 4$, until the iterates approach the new fixed point $\widetilde{\boldsymbol{S}}_{1 / 4}$, then reduce $\varepsilon$ again, etc. (Of course it would be wasteful to require too much accuracy of the fixed points prior to the final one.) In this way, one can be sure that the iterates are close to the classical solution $\widetilde{\boldsymbol{S}}$ when $\varepsilon$ is small, and that the early iterations will be accomplished very quickly. In the examples studied in $[\mathbf{9}, \mathbf{1 5}]$, the fixed point at $\varepsilon=1 / 10$ proved to be a good approximation to the free-boundary solution. Another reasonable possibility is to choose $\varepsilon_{n+1}=f\left(E_{n}\right)$ for each $n$, where $f$ denotes some empirically-chosen, positive, increasing function, and where $E_{n}$ denotes the maximum forward progression accomplished by the $n$-th iteration, divided by $\varepsilon_{n}$. Presumably, $E_{n}$ is a reasonable measure of the current error.

\section{Definitions and preliminary results.}

2.1. General notation and definitions. For any $x_{0} \in \mathbb{R}^{N}$ and $r \geq 0$, we define the open ball $B\left(x_{0} ; r\right)=\left\{x \in \mathbb{R}^{N}:\left|x-x_{0}\right|<r\right\}$ and closed ball $\bar{B}\left(x_{0} ; r\right)=\left\{x \in \mathbb{R}^{N}:\left|x-x_{0}\right| \leq r\right\}$. For $\Sigma, \Sigma_{0} \subset \mathbb{R}^{N}$ and $x_{0} \in \mathbb{R}^{N}$, we define $d\left(\Sigma, \Sigma_{0}\right)=\inf \left\{|x-y|: x \in \Sigma, y \in \Sigma_{0}\right\}$ and $d\left(x_{0}, \Sigma\right)=d\left(\left\{x_{0}\right\}, \Sigma\right)$. For any set $\Sigma$ and value $\alpha>0$, we use $\mathrm{Cl}(\Sigma)$ (resp. $\partial \Sigma, N_{\alpha}(\Sigma)$ ) to denote the closure (boundary, $\alpha$-neighborhood) of $\Sigma$. The term "null function" denotes a fixed but arbitrary continuous, strictly increasing function $z(t):[0, \infty) \rightarrow \mathbb{R}$ such that $z(0)=0$. 
2.2. Additional surface and multi-surface families and metrics. Let $\mathfrak{X}$ denote the family of all simple closed $(N-1)$-dimensional hypersurfaces $S$ in $\mathbb{R}^{N}$, and let $D(S)$ (resp. $E(S)$ ) denote the interior (exterior) complement of $S \in \mathfrak{X}$. The partial orderings " $<$ " and " $\leq$ " in $\mathfrak{X}$ are defined as in $\S 1.1$. For any values $0 \leq \Re_{0} \leq \Re_{1}<\infty$, let $\mathbb{X}\left(\Re_{0}, \Re_{1}\right)$ denote the family of all surfaces $S \in \mathbb{X}\left(\Re_{0}\right)$ such that $S \subset \bar{B}\left(0 ; \Re_{1}\right)$. For $0<\rho \leq \Re_{0}<\Re_{1}$, let $\mathbb{X}^{-}\left(\Re_{0}, \Re_{1} ; \rho\right)$ (resp. $\left.\mathbb{X}^{+}\left(\Re_{0}, \Re_{1} ; \rho\right)\right)$ denote the family of all surfaces $S \in \mathbb{X}\left(\Re_{0}, \Re_{1}\right)$ such that $S$ has an interior (resp. exterior) tangent ball of radius $\rho$ at every point. We also define $\mathbb{X}\left(\Re_{0}, \Re_{1} ; \rho\right)=$ $\mathbb{X}^{-}\left(\Re_{0}, \Re_{1} ; \rho\right) \cap \mathbb{X}^{+}\left(\Re_{0}, \Re_{1} ; \rho\right)$. For any $S_{1}, S_{2} \in \mathbb{X}\left(\Re_{0}, \Re_{1}\right)$, we use the notation $\min \left(S_{1}, S_{2}\right) \in \mathbb{X}\left(\Re_{0}, \Re_{1}\right)$ (resp. $\max \left(S_{1}, S_{2}\right) \in \mathbb{X}\left(\Re_{0}, \Re_{1}\right)$ ) to denote the boundary of the region $D\left(S_{1}\right) \cap D\left(S_{2}\right)$ (resp. $D\left(S_{1}\right) \cup D\left(S_{2}\right)$ ). Clearly, $\min \left(S_{1}, S_{2}\right) \in \mathbb{X}^{+}\left(\Re_{0}, \Re_{1} ; \rho\right)$ whenever $S_{1}, S_{2} \in \mathbb{X}^{+}\left(\Re_{0}, \Re_{1} ; \rho\right)$, and $\max \left(S_{1}, S_{2}\right) \in \mathbb{X}^{-}\left(\Re_{0}, \Re_{1} ; \rho\right)$ whenever $S_{1}, S_{2} \in \mathbb{X}^{-}\left(\Re_{0}, \Re_{1} ; \rho\right)$. If $S \in$ $\mathbb{X}^{ \pm}\left(\Re_{0}, \Re_{1} ; \rho\right)$, then $\alpha S \in \mathbb{X}^{ \pm}\left(\alpha \Re_{0}, \alpha \Re_{1} ; \alpha \rho\right)$ for any $\alpha>0$. For any $k \in \mathbb{N}$, we use $\mathbf{x}_{k}\left(\Re_{0}, \Re_{1}\right)$ (resp. $\quad \mathbf{x}_{k}^{ \pm}\left(\Re_{0}, \Re_{1} ; \rho\right), \mathbf{x}_{k}\left(\Re_{0}, \Re_{1} ; \rho\right)$ ) to denote the family of all multisurfaces $\boldsymbol{S}=\left(S_{1}, \ldots, S_{k}\right) \in \mathbb{X}^{k}\left(\Re_{0}, \Re_{1}\right)$ (resp. $\left.\left[\mathbb{X}^{ \pm}\left(\Re_{0}, \Re_{1} ; \rho\right)\right]^{k}, \mathbb{X}^{k}\left(\Re_{0}, \Re_{1} ; \rho\right)\right)$ such that $S_{i}<S_{i+1}$ for $i=1, \ldots, k-1$. For any $\boldsymbol{\lambda}=\left(\lambda_{1}, \ldots, \lambda_{k}\right) \in(1, \infty)^{k}$, we define the metric $\boldsymbol{M}_{\boldsymbol{\lambda}}$ in $\mathbf{X}_{k}(0)$ such that

$$
\boldsymbol{M}_{\boldsymbol{\lambda}}\left(\boldsymbol{S}_{1}, \boldsymbol{S}_{2}\right):=\max \left\{M\left(S_{1, i}, S_{2, i}\right) / \ln \left(\lambda_{i}\right): i=1,2, \ldots, k\right\}
$$

for any $\boldsymbol{S}_{1}=\left(S_{1,1}, \ldots, S_{1, k}\right), \boldsymbol{S}_{2}=\left(S_{2,1}, \ldots, S_{2, k}\right) \in \mathbf{x}_{k}(0)$, where

$$
M\left(S_{1}, S_{2}\right):=\inf \left\{\lambda \geq 0: \exp (-\lambda) S_{1} \leq S_{2} \leq \exp (\lambda) S_{1}\right\}
$$

for any $S_{1}, S_{2} \in \mathbb{X}(0)$. Observe that for any $\boldsymbol{\lambda} \in(1, \infty)^{k}$, the metric $\boldsymbol{M}_{\boldsymbol{\lambda}}$ is equivalent to the metric $\boldsymbol{M}$ of Eq. (1.7). Moreover, $\boldsymbol{M}_{e}=\boldsymbol{M}$, where $\boldsymbol{e}=(e, \ldots, e)$ and $e=\exp (1)$.

2.3. Definitions for the operator method. Given $S_{1}, S_{2} \in \mathfrak{X}$ such that $S_{1}<S_{2}$ or $S_{2}<S_{1}$, we use $\Omega\left(S_{1}, S_{2}\right)$ to denote the annular domain whose boundary is $S_{1} \cup S_{2}$. We use $U^{+}=U^{+}\left(S_{1}, S_{2} ; x\right)$ to denote the capacitary potential in (the closure of) $\Omega=\Omega\left(S_{1}, S_{2}\right)$, oriented such that $U^{+}(x)=0$ (resp. $U^{+}(x)=1$ ) on the inner (outer) boundary component of $\Omega\left(S_{1}, S_{2}\right)$. We also define $U^{-}\left(S_{1}, S_{2} ; x\right)=1-U^{+}\left(S_{1}, S_{2} ; x\right)$. Given $\varepsilon \in(0,1)$ and surfaces $S_{1}, S_{2} \in \mathfrak{X}$ with $S_{1}<S_{2}$ or $S_{2}<S_{1}$, we define

$$
\Phi_{\varepsilon}^{ \pm}\left(S_{1}, S_{2}\right):\left\{x \in \Omega\left(S_{1}, S_{2}\right): U^{ \pm}\left(S_{1}, S_{2} ; x\right)=\varepsilon\right\} \in \mathfrak{X} .
$$

Observe that for any $0 \leq \Re_{0}<\Re_{1}$, we have $\Phi_{\varepsilon}^{ \pm}\left(S_{1}, S_{2}\right) \in \mathbb{X}\left(\Re_{0}, \Re_{1}\right)$ whenever $S_{1}, S_{2} \in \mathbb{X}\left(\Re_{0}, \Re_{1}\right)$ (see [6, Lemma 3.3]). Clearly, the surface $\Phi_{\varepsilon}^{ \pm}\left(S_{1}, S_{2}\right)$ lies between the surfaces $S_{1}$ and $S_{2}$, and $\Phi_{\varepsilon}^{ \pm}\left(S_{1}, S_{2}\right)=\Phi_{1-\varepsilon}^{\mp}\left(S_{1}\right.$, $\left.S_{2}\right)$. For $\varepsilon \in(0,1)$ and any surfaces $S_{1}, S_{2} \in \mathfrak{X}$ with $S_{1}<S_{2}$, we define

$$
\Psi_{\varepsilon}\left(S_{1}, S_{2}\right):=\left\{x \in \Omega\left(S_{1}, S_{2}\right): f\left(x,\left(d\left(x, S_{1}\right) / \varepsilon\right),\left(d\left(x, S_{2}\right) / \varepsilon\right)\right)=0\right\} .
$$


Here, we define $f(x, p, q):=F(x, 1 / p, 1 / q)$, where $F(x, p, q): \mathbb{R}^{N} \times \mathbb{R}_{+} \times$ $\mathbb{R}_{+} \rightarrow \mathbb{R}$ denotes a given $C^{1}$-function satisfying Assumptions (A1)-(A3) for some given value $\boldsymbol{\Re}_{0} \geq 0$. For any $\Re_{1} \in\left[\Re_{0}, \infty\right)$, it follows from these properties that $\Psi_{\varepsilon}\left(S_{1}, S_{2}\right) \in \mathbb{X}\left(\Re_{0}, \Re_{1}\right)$ whenever $S_{1}, S_{2} \in \mathbb{X}\left(\Re_{0}, \Re_{1}\right)$ (see [6, Lemma 3.3]). Finally, for any $\Re_{1} \in\left[\Re_{0}, \infty\right), \varepsilon \in(0,1)$, and $\left(S^{-}, S, S^{+}\right) \in$ $\mathbf{X}_{3}\left(\Re_{0}, \Re_{1}\right)$, we define

$$
T_{\varepsilon}\left(S^{-}, S, S^{+}\right)=\Psi_{\varepsilon}\left(\Phi_{\varepsilon}^{-}\left(S^{-}, S\right), \Phi_{\varepsilon}^{+}\left(S, S^{+}\right)\right) \in \mathbb{X}\left(\Re_{0}, \Re_{1}\right) .
$$

Lemma 2.4. Let $S_{1}, S_{2}, \widehat{S}_{1}, \widehat{S}_{2} \in \mathfrak{X}$ denote surfaces such that $S_{1}<S_{2}$, $\widehat{S}_{1}<\widehat{S}_{2}, S_{1} \leq \widehat{S}_{1}$, and $S_{2} \leq \widehat{S}_{2}$. Then, for any $\varepsilon \in(0,1)$, we have:

(a) $\Phi_{\varepsilon}^{ \pm}\left(S_{1}, S_{2}\right) \leq \Phi_{\varepsilon}^{ \pm}\left(\widehat{S}_{1}, \widehat{S}_{2}\right)$, where the inequality is strict unless $S_{1}=\widehat{S}_{1}$ and $S_{2}=\widehat{S}_{2}$, and

(b) $\Phi_{\varepsilon}^{ \pm}\left(\lambda S_{1}, \lambda S_{2}\right)=\lambda \Phi_{\varepsilon}^{ \pm}\left(S_{1}, S_{2}\right)$ for any $\lambda>0$.

If actually $S_{1}, S_{2}, \widehat{S}_{1}, \widehat{S}_{2} \in \mathbb{X}(0)$, then, for any $\varepsilon \in(0,1)$, we have:

(c) $\Psi_{\varepsilon}\left(S_{1}, S_{2}\right) \leq \Psi_{\varepsilon}\left(\widehat{S}_{1}, \widehat{S}_{2}\right)$, where the inequality is strict if either $S_{1}<\widehat{S}_{1}$ or $S_{2}<\widehat{S}_{2}$

(d) $\Psi_{\varepsilon}\left(\lambda S_{1}, \lambda S_{2}\right) \geq \lambda \Psi_{\varepsilon}\left(S_{1}, S_{2}\right)$ for $0<\lambda \leq 1$, and

(e) $\Psi_{\varepsilon}\left(\lambda S_{1}, \lambda S_{2}\right) \leq \lambda \Psi_{\varepsilon}\left(S_{1}, S_{2}\right)$ for $\lambda \geq 1$.

Proof. See [6, Lemmas 3.8, 3.9, and 3.10].

Lemma 2.5. Let $\left(S^{-}, S, S^{+}\right)$and $\left(\widehat{S}^{-}, \widehat{S}, \widehat{S}^{+}\right)$be ordered triples in $\mathbf{X}_{3}(0)$. Then, for any $\varepsilon \in(0,1)$, we have:

(a) $T_{\varepsilon}\left(S^{-}, S, S^{+}\right) \leq T_{\varepsilon}\left(\widehat{S}^{-}, \widehat{S}, \widehat{S}^{+}\right)$if $S \leq \widehat{S}$ and $S^{ \pm} \leq \widehat{S}^{ \pm}$, where the inequality is strict unless $S=\widehat{S}$ and $S^{ \pm}=\widehat{S}^{ \pm}$,

(b) $T_{\varepsilon}\left(\lambda S^{-}, \lambda S, \lambda S^{+}\right) \geq \lambda T_{\varepsilon}\left(S^{-}, S, S^{+}\right)$for $0<\lambda \leq 1$,

(c) $T_{\varepsilon}\left(\lambda S^{-}, \lambda S, \lambda S^{+}\right) \leq \lambda T_{\varepsilon}\left(S^{-}, S, S^{+}\right)$for $\lambda \geq 1$, and

(d)

$$
\begin{aligned}
& M\left(T_{\varepsilon}\left(S^{-}, S, S^{+}\right), T_{\varepsilon}\left(\widehat{S}^{-}, \widehat{S}, \widehat{S}^{+}\right)\right) \\
& \leq \max \left\{M(S, \widehat{S}), M\left(S^{-}, \widehat{S}^{-}\right), M\left(S^{+}, \widehat{S}^{+}\right)\right\},
\end{aligned}
$$

in terms of the metric of Eq. (2.2).

Proof. Parts (a), (b), (c) follow from Lemma 2.4, and part (d) follows from parts (a), (c).

Remark 2.6. For each $\varepsilon \in(0,1)$, the mapping $\boldsymbol{T}_{\varepsilon}(\boldsymbol{S})=\boldsymbol{T}(\boldsymbol{S} ; \varepsilon): \mathbb{Y} \rightarrow \mathbb{Y}$ introduced in Eqs. (1.5), (1.6) is also defined by

$$
\begin{aligned}
\boldsymbol{T}_{\varepsilon}(\boldsymbol{S}) & =\boldsymbol{T}_{\varepsilon}\left(S_{1}, \ldots, S_{k}\right) \\
: & =\left(T_{\varepsilon, 1}\left(S_{0}, S_{1}, S_{2}\right), T_{\varepsilon, 2}\left(S_{1}, S_{2}, S_{3}\right), \ldots, T_{\varepsilon, k}\left(S_{k-1}, S_{k}, S_{k+1}\right)\right),
\end{aligned}
$$


where $S_{0}:=S_{*}^{-}, S_{k+1}:=S_{*}^{+}$, and, for each $i=1, \ldots, k$, the definition of $T_{\varepsilon, i}\left(S_{i-1}, S_{i}, S_{i+1}\right)$ coincides with the definition of $T_{\varepsilon}\left(S^{-}, S, S^{+}\right)$in $\S 2.3$, in the case where $S:=S_{i} \in \mathbb{X}\left(\Re_{0}\right), S^{ \pm}:=S_{i \pm 1} \in \mathbb{X}\left(\Re_{0}\right)$, and $F:=F_{i}$. As a direct consequence of Lemma 2.5(a), (d), the transformation $\boldsymbol{T}_{\varepsilon}$ is monotone and continuous, in the sense that $\boldsymbol{T}_{\varepsilon}\left(\boldsymbol{S}_{1}\right) \leq \boldsymbol{T}_{\varepsilon}\left(\boldsymbol{S}_{2}\right)$ whenever $\varepsilon \in(0,1)$ and $\boldsymbol{S}_{1} \leq \boldsymbol{S}_{2}$ in $\mathbb{Y}$, and $\boldsymbol{M}\left(\boldsymbol{T}_{\varepsilon}\left(\boldsymbol{S}_{1}\right), \boldsymbol{T}_{\varepsilon}\left(\boldsymbol{S}_{2}\right)\right) \leq \boldsymbol{M}\left(\boldsymbol{S}_{1}, \boldsymbol{S}_{2}\right)$ for all $\varepsilon \in(0,1)$ and $\boldsymbol{S}_{1}, \boldsymbol{S}_{2} \in \mathbb{Y}$.

\section{A three-similar-surface estimate.}

Theorem 3.1. In the context of $\S 2.3$, for fixed values $0<r \leq \Re_{0} \leq \Re_{1}$, there exist a value $\eta \in(0,1)$ and a strictly-positive function $g(t):(0, \eta] \rightarrow$ $\mathbb{R}_{+}$such that

$$
T_{\varepsilon}\left(P_{-} S, S, P_{+} S\right) \geq \exp \left[g\left(P_{+}-1\right) \varepsilon\right] S
$$

for all $S \in \mathbb{X}^{-}\left(\Re_{0}, \Re_{1} ; r\right)$, all $\varepsilon \in(0,1)$, and all values $P_{ \pm}>0$ with $0<$ $\pm\left(P_{ \pm}-1\right) \leq \eta$ and $\left(1-P_{-}\right) \leq g\left(P_{+}-1\right)$, and such that

$$
T_{\varepsilon}\left(P_{-} S, S, P_{+} S\right) \leq \exp \left[-g\left(1-P_{-}\right) \varepsilon\right] S
$$

for all $S \in \mathbb{X}^{+}\left(\Re_{0}, \Re_{1} ; r\right)$, all $\varepsilon \in(0,1)$, and all values $P_{ \pm}>0$ with $0<$ $\pm\left(P_{ \pm}-1\right) \leq \eta$ and $\left(P_{+}-1\right) \leq g\left(1-P_{-}\right)$.

Remark 3.2. Under the assumptions of Theorem 3.1, the assertion can be restated as follows: There exist a value $\eta_{0} \in(0,1)$ and a strictly-positive function $g_{0}(t):\left(0, \eta_{0}\right] \rightarrow \mathbb{R}_{+}$such that $T_{\varepsilon}(S, P S, P \widehat{P} S) \geq \exp \left[g_{0}(\widehat{P}-\right.$ 1) $\varepsilon] P S$ for all $S \in \mathbb{X}^{-}\left(\Re_{0}, \Re_{1} ; r\right), \varepsilon \in(0,1)$, and values $1<P \leq \widehat{P} \leq 1+\eta_{0}$ satisfying $(P-1) \leq g_{0}(\widehat{P}-1)$, and such that $T_{\varepsilon}(P \widehat{P} S, P S, S) \leq \exp \left[-g_{0}(1-\right.$ $\widehat{P}) \varepsilon] P S$ for all $S \in \mathbb{X}^{+}\left(\Re_{0}, \Re_{1} ; r\right), \varepsilon \in(0,1)$, and values $1-\eta_{0} \leq \widehat{P} \leq P<1$ satisfying $(1-P) \leq g_{0}(1-\widehat{P})$.

Lemma 3.3. In the context of $\S 2.3$ :

(a) For any $x \in \mathbb{R}^{N}$ and $\lambda \in \mathbb{R}_{+}$, there exists one and only one pair $(\alpha, \beta) \in \mathbb{R}_{+}^{2}$ such that $f(x, \alpha, \beta)=0$ and $\alpha+\beta=\lambda$.

(b) For a given compact set $K \subset \mathbb{R}^{N}$, let $A(\lambda, \mu):=\sup (Q(\lambda, \mu))$ for any $0<\lambda \leq \mu<\infty$, where $\Sigma(\lambda, \mu)$ denotes the set of all ordered pairs $(\alpha, \beta) \in \mathbb{R}_{+}^{2}$ such that $\lambda \leq \alpha+\beta \leq \mu$ and $f(x, \alpha, \beta)=0$ for some $x \in K$, and where $Q(\lambda, \mu):=\{(\beta / \alpha),(\alpha / \beta):(\alpha, \beta) \in \Sigma(\lambda, \mu)\} \neq \emptyset$.

Then $0<A(\lambda, \mu) \leq A\left(\lambda^{\prime}, \mu^{\prime}\right)<\infty$ whenever $0<\lambda^{\prime} \leq \lambda \leq \mu \leq \mu^{\prime}<\infty$. It follows that $\alpha \leq A(\lambda, \mu) \beta, \beta \leq A(\lambda, \mu) \alpha$, and

$$
\lambda /(1+A(\lambda, \mu)) \leq \alpha, \beta \leq \mu A(\lambda, \mu) /(1+A(\lambda, \mu))
$$

for any $(x, \alpha, \beta) \in K \times \mathbb{R}_{+}^{2}$ such that $\lambda \leq \alpha+\beta \leq \mu$ and $f(x, \alpha, \beta)=0$. 
Proof. These results follow easily from Assumptions (A1), (A3).

Lemma 3.4. In the context of $\S 2.3$, let $\Re_{1} \in \mathbb{R}_{+}$be given. Then

$$
d\left(\Psi_{\varepsilon}\left(S^{-}, S^{+}\right), S^{ \pm}\right) \geq \delta_{0} /\left(1+A\left[\left(\delta_{0} / \varepsilon\right),\left(2 \rho_{0} / \varepsilon\right)\right]\right)
$$

uniformly for all values $\varepsilon \in(0,1)$ and $0<\delta_{0}<\rho_{0}$, and over all surfaces $S^{ \pm} \in \mathbb{X}\left(0, \Re_{1}\right)$ such that $d\left(S^{-}, S^{+}\right) \geq \delta_{0}$ and $d\left(x, S^{ \pm}\right) \leq \rho_{0}$ for all $x \in$ $\Omega\left(S^{-}, S^{+}\right)$.

Proof. Under the assumptions, we have $\delta_{0} \leq d\left(S^{-}, S^{+}\right) \leq d\left(x, S^{-}\right)+$ $d\left(x, S^{+}\right) \leq 2 \rho_{0}$ for all $x \in \Omega\left(S^{-}, S^{+}\right)$. Therefore, the assertion follows from Lemma 3.3(b), where we set $\alpha=d\left(x, S^{-}\right), \beta=d\left(x, S^{+}\right), K=\bar{B}\left(0 ; \Re_{1}\right)$, $\lambda=\delta_{0} / \varepsilon$, and $\mu=2 \rho_{0} / \varepsilon$.

Lemma 3.5. Given the values $0<a_{0}<b_{0}$, there exist fixed values $A_{0}^{ \pm}>0$ such that

$$
\Phi_{\varepsilon}^{ \pm}\left(S(r), S\left(p_{ \pm} r\right)\right) \geq S\left(p_{\varepsilon}^{ \pm} r\right)
$$

for all $\varepsilon \in(0,1)$ and all values $r, p_{ \pm}>0$ such that $a_{0} \leq p_{-} r<r<p_{+} r \leq b_{0}$, where $p_{\varepsilon}^{ \pm}:=1+A_{0}^{ \pm}\left(p_{ \pm}-1\right) \varepsilon$ and $S(r):=\partial B(0 ; r)$ for all $r>0$.

Proof. Explicit calculations involving spherically-symmetric solutions of the Laplace equation show that

$$
\Phi_{\varepsilon}^{ \pm}\left(S(r), S\left(p_{ \pm} r\right)\right)=S\left(\phi_{\varepsilon}^{ \pm} r\right)
$$

where we define $\phi_{\varepsilon}^{ \pm}=\phi_{\varepsilon}^{ \pm}\left(p_{ \pm}\right)$such that $\phi_{\varepsilon}^{ \pm}=p_{ \pm}^{\varepsilon}$ for $N=2$, and $\phi_{\varepsilon}^{ \pm}=$ $\left(1+\left[p_{ \pm}^{2-N}-1\right] \varepsilon\right)^{1 /(2-N)}$ for $N \geq 3$. Further explicit calculations show that

$$
\phi_{\varepsilon}^{ \pm} \geq p_{\varepsilon}^{ \pm}:=1+A_{0}^{ \pm}\left(p_{ \pm}-1\right) \varepsilon
$$

whenever $\left(a_{0} / b_{0}\right) \leq p_{-} \leq 1 \leq p_{+} \leq\left(b_{0} / a_{0}\right)$ and $0<\varepsilon<1$, where we define $A_{0}^{ \pm}=\left(a_{0} / b_{0}\right)^{ \pm(N-1)}$ for $N \geq 2$.

Lemma 3.6. Let the values $0<\Re_{0}<\Re_{1}$ be given. Then for any $S \in$ $\mathbb{X}\left(\Re_{0}, \Re_{1}\right)$, we have:

$(3.7 \mathrm{a}, \mathrm{b}) \Re_{0}|t-1| \leq d(t x, S) \leq \Re_{1}|t-1| ; \Re_{0}|t-1| \leq d(x, t S) \leq \Re_{1}|t-1|$ for $x \in S$ and $t>0$, and we have

$$
\Re_{0}|t-1| \leq d(x, t S)-d(x, S) \leq \Re_{1}|t-1|
$$

for all $x \in \mathrm{Cl}(D(S))$ and $t \geq 1$, and for all $x \in \mathrm{Cl}(E(S))$ and $t \in(0,1]$. It follows from (3.8) that $\Re_{0}|t-1| \leq d(t x, S)-t d(x, S) \leq \Re_{1}|t-1|$ for $x \in D(S)$ and $t \in(0,1]$, and for $x \in E(S)$ and $t \geq 1$. 
Proof. The inequalities (3.7a) follow easily from the properties of the surfaces $S \in \mathbb{X}\left(\Re_{0}, \Re_{1}\right)$. The inequalities (3.7b) follow from (3.7a) via the identity $d(x, t S)=t d((x / t), S)$. For any $x \in D(S)$ and $t \geq 1$, it follows from (3.7b) that

$$
d(x, t S) \leq d(x, S)+d(y, t S) \leq d(x, S)+\Re_{1}(t-1),
$$

where $y \in S$ is a point such that $|y-x|=d(x, S)$. It also follows from (3.7b) that

$$
d(x, t S)=|x-y|+|y-z| \geq d(x, S)+d(y, t S) \geq d(x, S)+\Re_{0}(t-1)
$$

for any given $x \in D(S)$ and $t \geq 1$, where $y \in S$ and $z \in t S$ are chosen such that $|z-x|=d(x, t S)$ and such that the points $x, y, z$ are colinear. This completes the proof of (3.8) in the case where $x \in D(S)$ and $t \geq 1$. The proof of the alternate case is similar.

3.7. Proof of Theorem 3.1. We only prove the estimate (3.1), since the proof of (3.2) is analogous. Let the surface $S \in \mathbb{X}^{-}\left(\Re_{0}, \Re_{1} ; r\right)$ be fixed. We have that $D(S)=\cup_{x \in \Sigma} B(x ; r)$, where $\Sigma$ denotes the set of all $x \in \mathbb{R}^{N}$ such that $B(x ; r) \subset D(S)$. For any $t>0$, let $S(t):=\partial N_{t r}(\Sigma)=$ the boundary of the $t r$-neighborhood of $\Sigma$. Thus $S(1)=S$. For all $t>1-\left(r / \Re_{1}\right)$, we have

$$
S\left(1+h_{ \pm}(t-1)\right) \leq t S \leq S\left(1+h_{\mp}(t-1)\right) \quad \text { when } \quad \pm(t-1)>0,
$$

by Lemma 3.6, where $h_{+}:=\left(\Re_{0} / r\right)$ and $h_{-}:=\left(\Re_{1} / r\right)$. Therefore

$$
P_{ \pm} S \geq S\left(p_{ \pm}\right)
$$

for $0< \pm\left(P_{ \pm}-1\right)<\eta_{0}:=\left(r / \Re_{1}\right)$, where $p_{ \pm}:=1+h_{ \pm}\left(P_{ \pm}-1\right)$. It follows from (3.10) that for any $x_{0} \in \Sigma$, we have $\partial B\left(x_{0} ; p_{ \pm} r\right) \leq P_{ \pm} S$ under the same assumptions. Choose $\eta_{1} \in\left(0, \eta_{0}\right]$ such that $(1 / 2) \leq p_{-}<1<p_{+} \leq 2$ whenever $0< \pm\left(P_{ \pm}-1\right) \leq \eta_{1}$. Then, for $0< \pm\left(P_{ \pm}-1\right) \leq \eta_{1}$, Lemmas 2.4(a) and 3.5 (with $a_{0}=r / 2, b_{0}=2 r$ ) imply that

$$
\Phi_{\varepsilon}^{ \pm}\left(S, P_{ \pm} S\right) \geq \Phi_{\varepsilon}^{ \pm}\left(\partial B\left(x_{0} ; r\right), \partial B\left(x_{0} ; p_{ \pm} r\right)\right) \geq \partial B\left(x_{0} ; p_{\varepsilon}^{ \pm} r\right)
$$

for all $\varepsilon \in(0,1)$, where $p_{\varepsilon}^{ \pm}:=1+A_{0}^{ \pm}\left(p_{ \pm}-1\right) \varepsilon=1+A_{0}^{ \pm} h_{ \pm}\left(P_{ \pm}-1\right) \varepsilon$. Since $x_{0}$ is arbitrary in $\Sigma$, it follows from (3.11) that $\Phi_{\varepsilon}^{ \pm}\left(S, P_{ \pm} S\right) \geq S\left(p_{\varepsilon}^{ \pm}\right)$for $0< \pm\left(P_{ \pm}-1\right) \leq \eta_{2}$, where $\eta_{2} \in\left(0, \eta_{1}\right]$ is small enough so that $p_{\varepsilon}^{-}>0$. It follows by Lemma 2.4(c) that

$$
T_{\varepsilon}\left(P_{-} S, S, P_{+} S\right) \geq \Psi_{\varepsilon}\left(S\left(p_{\varepsilon}^{-}\right), S\left(p_{\varepsilon}^{+}\right)\right)
$$

for $0< \pm\left(P_{ \pm}-1\right) \leq \eta_{2}$ and $\varepsilon \in(0,1)$. Let $\alpha=\alpha\left(P_{-}, P_{+}\right):=\left(\left(p_{\varepsilon}^{+}-p_{\varepsilon}^{-}\right) / \varepsilon\right)=$ $\alpha_{+}+\alpha_{-}$, where $\alpha_{ \pm}=\alpha_{ \pm}\left(P_{ \pm}\right):= \pm\left(\left(p_{\varepsilon}^{ \pm}-1\right) / \varepsilon\right)= \pm A_{0}^{ \pm} h_{ \pm}\left(P_{ \pm}-1\right)>0$. For $0< \pm\left(P_{ \pm}-1\right) \leq \eta_{2}$ and $\varepsilon \in(0,1)$, we have

$$
d\left(S\left(p_{\varepsilon}^{-}\right), S\left(p_{\varepsilon}^{+}\right)\right) \geq \alpha r \varepsilon \geq\left(p_{\varepsilon}^{+}-1\right) r=\alpha_{+} r \varepsilon,
$$

$$
d\left(x, S\left(p_{\varepsilon}^{-}\right)\right) \leq \alpha r \varepsilon \quad \text { for all } x \in \Omega\left(S\left(p_{\varepsilon}^{-}\right), S\left(p_{\varepsilon}^{+}\right)\right) .
$$


In view of (3.14), Lemma 3.6 implies $S\left(p_{\varepsilon}^{+}\right) \leq\left[1+\left(\alpha r \varepsilon / \Re_{0}\right)\right] S\left(p_{\varepsilon}^{-}\right)$, and then that

$$
d\left(x, S\left(p_{\varepsilon}^{+}\right)\right) \leq d\left(x,\left[1+\left(\alpha r \varepsilon / \Re_{0}\right)\right] S\left(p_{\varepsilon}^{-}\right)\right) \leq\left(\Re_{1} / \Re_{0}\right) \alpha r \varepsilon
$$

for all $x \in \Omega\left(S\left(p_{\varepsilon}^{-}\right), S\left(p_{\varepsilon}^{+}\right)\right)$. Under the assumptions that $0< \pm\left(P_{ \pm}-1\right) \leq \eta_{2}$ and $\alpha_{-}<\alpha_{+}$, it follows from (3.13), (3.14), (3.15), and Lemma 3.4 that

$$
d\left(\Sigma, \Psi_{\varepsilon}\left(S\left(p_{\varepsilon}^{-}\right), S\left(p_{\varepsilon}^{+}\right)\right)\right)=d\left(\Sigma, S\left(p_{\varepsilon}^{-}\right)\right)+d\left[S\left(p_{\varepsilon}^{-}\right), \Psi_{\varepsilon}\left(S\left(p_{\varepsilon}^{-}\right), S\left(p_{\varepsilon}^{+}\right)\right)\right]
$$

for all $\varepsilon \in(0,1)$, where we define

$$
0<\phi\left(\alpha_{+}\right):=\left(\alpha_{+} / 2\left[1+A\left(\alpha_{+} r, 4\left(\Re_{1} / \Re_{0}\right) \alpha_{+} r\right)\right]\right)<\left(\alpha_{+} / 2\right),
$$

and where we have used the fact that $d\left(\Sigma, S\left(p_{\varepsilon}^{-}\right)\right)=p_{\varepsilon}^{-} r=\left(1-\alpha_{-} \varepsilon\right) r$. Let

$$
\begin{aligned}
& g_{1}\left(P_{+}-1\right):=\left(r / \Re_{1} A_{0}^{-}\right) \phi\left(\alpha_{+}\left(P_{+}\right)\right), \\
& g_{2}\left(P_{+}-1\right):=\left(C_{0} r / \Re_{1}\right) \phi\left(\alpha_{+}\left(P_{+}\right)\right),
\end{aligned}
$$

where $C_{0}:=\min \left\{1 / 3,\left(2 \Re_{1} / \Re_{0} A_{0}^{+} \eta_{2}\right)\right\}$ (so that $(1+t) \geq \exp \left(C_{0} t\right)$ whenever $0 \leq t \leq\left(A_{0}^{+} \Re_{0} \eta_{2} / 2 \Re_{1}\right)$, where the latter value is an upper bound for $\left(r \phi\left(\alpha_{+}\right) / \Re_{1}\right)$ over all $\left.P_{+} \in\left(1, \eta_{2}\right]\right)$. Then

$$
\begin{aligned}
T_{\varepsilon}\left(P_{-} S, S, P_{+} S\right) & \geq\left(1+\left(r / \Re_{1}\right)\left(2 \phi\left(\alpha_{+}\right)-\alpha_{-}\right) \varepsilon\right) S \\
& \geq\left(1+\left(r / \Re_{1}\right) \phi\left(\alpha_{+}\right) \varepsilon\right) S \\
& =\left(1+\left[g_{2}\left(P_{+}-1\right) / C_{0}\right] \varepsilon\right) S \\
& \geq \exp \left[g_{2}\left(P_{+}-1\right) \varepsilon\right] S
\end{aligned}
$$

whenever $0< \pm\left(P_{ \pm}-1\right) \leq \eta_{2},\left(1-P_{-}\right) \in\left(0, g_{1}\left(P_{+}-1\right)\right]$, and $\varepsilon \in(0,1)$, where the first inequality in (3.17) follows from (3.9), (3.12), and (3.16). To complete the proof of (3.1), we define $g(t):=\min \left\{g_{1}(t), g_{2}(t)\right\}$, and observe that all the above estimates are independent of $S \in \mathbb{X}^{-}\left(\Re_{0}, \Re_{1} ; r\right)$.

\section{Variational estimates for capacitary potentials and their boundary derivatives.}

Theorem 4.1. Given the values $0<\Re_{0} \leq \Re_{1}, 0<\mu_{0}<1<\mu_{1}, \varepsilon \in(0,1)$, and $P>1$, there exist constants $0<C_{0} \leq C_{1}$ such that

$$
\exp \left(C_{0}(\beta-\alpha)\right) \leq\left[U^{+}\left(S_{1}, \alpha S_{2} ; x\right) / U^{+}\left(S_{1}, \beta S_{2} ; x\right)\right] \leq \exp \left(C_{1}(\beta-\alpha)\right)
$$

uniformly for all $\alpha, \beta \in\left[\mu_{0}, \mu_{1}\right]$ and $S_{1}, S_{2} \in \mathbb{X}\left(\Re_{0}, \Re_{1}\right)$ such that $\alpha<\beta$, $P S_{1}<S_{2}$, and $P S_{1}<\alpha S_{2}$, and for all $x \in \Omega\left(S_{1}, \Phi_{\varepsilon}^{+}\left(S_{1}, \alpha S_{2}\right)\right)$. Also,

(4.2) $\exp \left(C_{0}(\beta-\alpha)\right) \leq\left[U^{-}\left(\beta S_{1}, S_{2} ; x\right) / U^{-}\left(\alpha S_{1}, S_{2} ; x\right)\right] \leq \exp \left(C_{1}(\beta-\alpha)\right)$ uniformly for all $\alpha, \beta \in\left[\mu_{0}, \mu_{1}\right]$ and $S_{1}, S_{2} \in \mathbb{X}\left(\Re_{0}, \Re_{1}\right)$ such that $\alpha<\beta$, $P S_{1}<S_{2}$, and $P \beta S_{1}<S_{2}$, and for all $x \in \Omega\left(\Phi_{\varepsilon}^{-}\left(\beta S_{1}, S_{2}\right), S_{2}\right)$. 
Corollary 4.2. In the context of Theorem 4.1, under the assumptions for (4.1), we have

$$
\begin{aligned}
\exp \left(C_{0}(\beta-\alpha)\right) & \leq\left[\left|\nabla U^{+}\left(S_{1}, \alpha S_{2} ; x\right)\right| /\left|\nabla U^{+}\left(S_{1}, \beta S_{2} ; x\right)\right|\right] \\
& \leq \exp \left(C_{1}(\beta-\alpha)\right)
\end{aligned}
$$

for all $x \in S_{1}$ at which the derivatives exist. Under the assumptions for (4.2), we have

$$
\begin{aligned}
\exp \left(C_{0}(\beta-\alpha)\right) & \leq\left[\left|\nabla U^{-}\left(\beta S_{1}, S_{2} ; x\right)\right| /\left|\nabla U^{-}\left(\alpha S_{1}, S_{2} ; x\right)\right|\right] \\
& \leq \exp \left(C_{1}(\beta-\alpha)\right)
\end{aligned}
$$

for all $x \in S_{2}$ at which the derivatives exist.

Theorem 4.3. Given the values $0<\rho<\Re_{0}<\Re_{1}, 0<P_{-}<1<P_{+}$, and $0<\mu_{0}<1<\mu_{1}$, let $\left(S^{-}, S, S^{+}\right) \in \mathbf{X}_{3}\left(\Re_{0}, \Re_{1}\right)$ denote a fixed ordered triple such that $S \in \mathbb{X}\left(\Re_{0}, \Re_{1} ; \rho\right)$ and $S^{-}<P_{-} S<S<P_{+} S<S^{+}$. For any $\mu>0$, we define the surfaces

$$
S^{-}(\mu)=\min \left(P_{-} S, \mu S^{-}\right) ; S^{+}(\mu)=\max \left(P_{+} S, \mu S^{+}\right) .
$$

Let $Q^{ \pm}(x)=\left(1 /\left|\nabla U^{ \pm}(x)\right|\right)$ on $S$, where $U^{ \pm}(x)=U^{ \pm}\left(S, S^{ \pm} ; x\right)$. Also let $Q^{ \pm}(\mu ; x)=\left(1 /\left|\nabla U_{\mu}^{ \pm}(x)\right|\right)$ for any $x \in S$ and $\mu>0$, where $U_{\mu}^{ \pm}(x)=$ $U^{ \pm}\left(S, S^{ \pm}(\mu) ; x\right)$ in the closure of the domain $\Omega^{ \pm}(\mu):=\Omega\left(S, S^{ \pm}(\mu)\right)$. Then there exist constants $0<A_{0} \leq A_{1}$ such that

$$
A(1 / \mu) \ln (\mu) \leq Q^{+}(\mu ; x)-Q^{+}(x) \leq A(\mu) \ln (\mu)
$$

uniformly for all $x \in S$ and $\mu \in\left[\mu_{0}, \mu_{1}\right]$, and

$$
A(\mu) \ln (1 / \mu) \leq Q^{-}(\mu ; x)-Q^{-}(x) \leq A(1 / \mu) \ln (1 / \mu)
$$

uniformly for all $x \in S$ and $\mu \in\left[\mu_{0}, \mu_{1}\right]$. Here, we define $A(\mu)=A_{0}>0$ for $\mu \in\left[\mu_{0}, 1\right]$ and $A(\mu)=A_{1} \geq A_{0}$ for $\mu \in\left(1, \mu_{1}\right]$.

Lemma 4.4. Given $0<\Re_{0}<\Re_{1}, P>1$, and $0<\alpha<\beta<1$, there exists a value $\lambda>1$ such that $\Phi_{\alpha}^{+}\left(S_{1}, \lambda S_{2}\right) \leq \Phi_{\beta}^{+}\left(S_{1}, S_{2}\right)$ for all $S_{1}, S_{2} \in \mathbb{X}:=$ $\mathbb{X}\left(\Re_{0}, \Re_{1}\right)$ such that $S_{2} \geq P S_{1}$.

Proof. If the assertion is false, then there exist sequences $\left(S_{1, n}\right),\left(S_{2, n}\right)$ of surfaces in $\mathbb{X}$ and a sequence $\left(\lambda_{n}\right)$ of values in $(1, \infty)$ such that $\lambda_{n} \downarrow 1$ as $n \rightarrow \infty$, and such that for each $n \in \mathbb{N}$, it is true that $S_{2, n} \geq P S_{1, n}$, but it is not true that $\Phi_{\alpha}^{+}\left(S_{1, n}, \lambda_{n} S_{2, n}\right) \leq \Phi_{\beta}^{+}\left(S_{1, n}, S_{2, n}\right)$. Now the polar-coordinate representations of surfaces in $\mathbb{X}$ are equicontinuous and uniformly bounded. Therefore, any sequence in $\mathbb{X}$ has a convergent subsequence, due to the theorem of Ascoli-Arzela. By passing to a subsequence (still indexed by $n \in \mathbb{N}$ ) if necessary, we can assume that $S_{1, n} \rightarrow S_{1} \in \mathbb{X}$ and $S_{2, n} \rightarrow S_{2} \in \mathbb{X}$ as $n \rightarrow \infty$, where $S_{2} \geq P S_{1}$. Clearly $\Phi_{\alpha}^{+}\left(S_{1}, S_{2}\right)<\Phi_{\beta}^{+}\left(S_{1}, S_{2}\right)$, and it is easily seen that $\Phi_{\alpha}^{+}\left(S_{1, n}, \lambda_{n} S_{2, n}\right) \rightarrow \Phi_{\alpha}^{+}\left(S_{1}, S_{2}\right)$ and $\Phi_{\beta}^{+}\left(S_{1, n}, S_{2, n}\right) \rightarrow$ 
$\Phi_{\beta}^{+}\left(S_{1}, S_{2}\right)$, both as $n \rightarrow \infty$. Therefore, $\Phi_{\alpha}^{+}\left(S_{1, n}, \lambda_{n} S_{2, n}\right)<\Phi_{\beta}^{+}\left(S_{1, n}, S_{2, \mathrm{n}}\right)$ for all sufficiently large $n \in \mathbb{N}$, contradicting our choice of the sequences $\left(S_{1, n}\right),\left(S_{2, n}\right)$, and $\left(\lambda_{n}\right)$. Therefore the assertion holds.

Lemma 4.5. Let $0<\Re_{0}<\Re_{1}$ and $P>1$ be given. Then:

(a) For any $\alpha, \beta \in[0,1]$ such that $\alpha<\beta$, there exists a value $d_{0}>0$ such that $d\left(\Phi_{\alpha}^{+}\left(S_{1}, S_{2}\right), \Phi_{\beta}^{+}\left(S_{1}, S_{2}\right)\right) \geq d_{0}$ for all $S_{1}, S_{2} \in \mathbb{X}:=\mathbb{X}\left(\Re_{0}, \Re_{1}\right)$ such that $S_{2} \geq P S_{1}$.

(b) For any $\alpha, \beta \in(0,1)$ with $\alpha \leq \beta$, the derivative $\left|\nabla U^{+}\left(S_{1}, S_{2} ; x\right)\right|$ is bounded above, uniformly over all $S_{1}, S_{2} \in \mathbb{X}$ such that $P S_{1}<S_{2}$, and over all $x \in \Omega\left(S_{1}, S_{2}\right)$ such that $\alpha \leq U^{+}\left(S_{1}, S_{2} ; x\right) \leq \beta$.

Proof. For any surfaces $S_{1}, S_{2} \in \mathbb{X}$ such that $S_{2} \geq P S_{1}$, we have $S_{1}\left(x_{0}\right) \leq$ $S_{1}$ and $S_{2}\left(x_{0}\right) \leq S_{2}$ for all $x_{0} \in S_{1}$. Here, we define $S_{1}\left(x_{0}\right)=\partial H\left(x_{0}\right)$ and $S_{2}\left(x_{0}\right)=\partial N_{\delta}\left(H\left(x_{0}\right)\right)$ for all $x_{0} \in \mathfrak{A}:=B\left(0 ; \Re_{1}\right) \backslash B\left(0 ; \Re_{0}\right)$, where $\delta=\Re_{0}(P-1)$ and $H\left(x_{0}\right)$ denotes the convex hull of the set $\left\{x_{0}\right\} \cup \bar{B}\left(x_{1} ; \mu\right)$, where $x_{1}=\left(1-\left(\Re_{0} /\left|x_{0}\right|\right)\right) x_{0}$ and $\mu=\Re_{0}^{2} / \Re_{1}$. For given $\alpha \in(0,1)$, we have $d_{0}(\alpha):=d\left(S_{1}\left(x_{0}\right), \Phi_{\alpha}^{+}\left(S_{1}\left(x_{0}\right), S_{2}\left(x_{0}\right)\right)\right)>0$ for all $x_{0} \in \mathfrak{A}$, due to the congruence of the annular domains $\Omega\left(S_{1}\left(x_{0}\right), S_{2}\left(x_{0}\right)\right), x_{0} \in \mathfrak{A}$. Since $\Phi_{\alpha}^{+}\left(S_{1}, S_{2}\right) \geq \Phi_{\alpha}^{+}\left(S_{1}\left(x_{0}\right), S_{2}\left(x_{0}\right)\right)$ for all $x_{0} \in S_{1}$ by Lemma 2.4(a), it follows that $d\left(S_{1}, \Phi_{\alpha}^{+}\left(S_{1}, S_{2}\right)\right) \geq d_{0}(\alpha)$ for all $S_{1}, S_{2} \in \mathbb{X}$ such that $S_{2} \geq$ $P S_{1}$. One can show analogously that $d\left(S_{2}, \Phi_{\alpha}^{+}\left(S_{1}, S_{2}\right)\right) \geq d_{1}(\alpha)>0$ for $\alpha \in(0,1)$ and for all $S_{1}, S_{2} \in \mathbb{X}$ such that $S_{2} \geq P S_{1}$. Part (b) follows easily from these estimates, in view of the fact that $\left|\nabla U^{+}\left(S_{1}, S_{2} ; x\right)\right| \leq$ $N / \min \left\{d\left(S_{1}, \Phi_{\alpha}^{+}\left(S_{1}, S_{2}\right)\right), d\left(S_{2}, \Phi_{\beta}^{+}\left(S_{1}, S_{2}\right)\right)\right\}$ for any $S_{1}, S_{2} \in \mathbb{X}$ such that $S_{1}<S_{2}$, and any $x \in \Omega\left(S_{1}, S_{2}\right)$ such that $\alpha \leq U^{+}\left(S_{1}, S_{2} ; x\right) \leq \beta$ (see [14, Thm. 2.10]). Part (a) follows from this.

Lemma 4.6. Given $0<\Re_{0}<\Re_{1}$ and $P>1$, there exist constants $0<$ $m \leq M$ such that

$$
m \leq \int_{S_{i}}\left|\nabla U^{+}\left(S_{1}, S_{2} ; x\right)\right|\left(x \cdot \nabla U^{+}\left(S_{1}, S_{2} ; x\right)\right) d \sigma \leq M
$$

for $i=1,2$, uniformly for all $C^{2}$-surfaces $S_{1}, S_{2} \in \mathbb{X}:=\mathbb{X}\left(\Re_{0}, \Re_{1}\right)$ such that $S_{2} \geq P S_{1}$, where $d \sigma$ denotes differential area of the $(N-1)$-dimensional hypersurface.

Proof. Given the $C^{2}$-surfaces $S_{1}<S_{2}$ in $\mathbb{X}$, let $U(x)=U^{+}\left(S_{1}, S_{2} ; x\right)$ in $\Omega:=\Omega\left(S_{1}, S_{2}\right)$. By the Cauchy-Schwarz inequality, we have

$$
\begin{aligned}
\int_{S_{i}}|\nabla U(x)|(x \cdot \nabla U(x)) d \sigma & =\int_{S_{i}}|\nabla U(x)|^{2}(x \cdot \nu) d \sigma \\
& \geq\left(\left(\int_{S_{i}}|\nabla U(x)|(x \cdot \nu) d \sigma\right)^{2} / \int_{S_{i}}(x \cdot \nu) d \sigma\right)
\end{aligned}
$$


for $i=1,2$, where $\nu$ denotes the exterior normal to $S_{i}$ (thus $\nu=\nabla U /|\nabla U|$ ). However, it follows from $S_{i} \in \mathbb{X}$ that $x \cdot \nu \geq \Re_{0}$ for all $x \in S_{i}, i=1,2$. Also, we have

$$
K\left(S_{1}, S_{2}\right) \geq K\left(\partial B\left(0 ; \Re_{0}\right), \partial B\left(0 ; \Re_{1}\right)\right)>0
$$

uniformly for all $S_{1}, S_{2} \in \mathbb{X}$ with $S_{1}<S_{2}$, where $K\left(S_{1}, S_{2}\right):=\int_{S_{1}}|\nabla U| d \sigma=$ $\int_{S_{2}}|\nabla U| d \sigma$ denotes the capacity of the annular domain $\Omega=\Omega\left(S_{1}, S_{2}\right)$. Also, it follows easily by the divergence theorem that

$$
\int_{S_{i}}(x \cdot \nu) d \sigma \leq\left(2 \pi^{N / 2} / \Gamma(N / 2)\right) \Re_{1}^{N}
$$

for $i=1,2$, where $\Gamma(\cdot)$ denotes the gamma function and $\left(2 \pi^{N / 2} / N \Gamma(N / 2)\right)$ is the volume of the unit ball in $\mathbb{R}^{N}$. In view of (4.10) and (4.11), it follows from (4.9) that

$$
\begin{aligned}
& \int_{S_{i}}|\nabla U(x)|(x \cdot \nabla U(x)) d \sigma \\
& \geq \Gamma(N / 2) \Re_{0}^{2} K^{2}\left(\partial B\left(0 ; \Re_{0}\right), \partial B\left(0 ; \Re_{1}\right)\right) /\left(2 \pi^{N / 2} \Re_{1}^{N}\right),
\end{aligned}
$$

for $i=1,2$, which establishes the uniform lower bound asserted in (4.8). To establish the uniform upper bound in (4.8) in the case where $i=2$, we first observe that $\widehat{U}(x)=2 U(x)-1$ in $\widehat{\Omega}:=\Omega\left(\widehat{S}, S_{2}\right)$, where $\widehat{S}=\{U=1 / 2\}$ and $\widehat{U}$ is the capacitary potential in $\widehat{\Omega}$. Thus, by Lemma $4.5(\mathrm{~b})$, there exists a constant $C_{0}>0$ such that

$$
|\nabla \widehat{U}(x)|=2|\nabla U(x)| \leq C_{0}
$$

for all $x \in \widehat{S}$, independent of admissible $S_{1}, S_{2} \in \mathbb{X}$. By a direct scaling argument, we have $K\left(t \widehat{S}, t S_{2}\right)=t^{N-2} K\left(\widehat{S}, S_{2}\right)$ for all $t>0$, where $K\left(t \widehat{S}, t S_{2}\right)$ denotes the capacity of the domain $t \widehat{\Omega}=\Omega\left(t \widehat{S}, t S_{2}\right)$. For any admissible $S_{1}, S_{2} \in \mathbb{X}$, it follows by application of the well-known Poincaré variational formula for capacity that

$$
\begin{aligned}
0 & \leq(N-2) K\left(\widehat{S}, S_{2}\right)=\left((d / d t) K\left(t \widehat{S}, t S_{2}\right)\right)_{t=1} \\
& =\int_{\widehat{S}}|\nabla \widehat{U}(x)|(x \cdot \nabla \widehat{U}(x)) d \sigma-\int_{S_{2}}|\nabla \widehat{U}(x)|(x \cdot \nabla \widehat{U}(x)) d \sigma .
\end{aligned}
$$

The uniform upper bound follows.

4.7. Proof of Theorem 4.1. We will only prove (4.1), since the proof of (4.2) is analogous. For given $\varepsilon \in(0,1)$, let $\varepsilon_{i}=\varepsilon+(i / 6) \eta$ for $i=1, \ldots, 5$, where $\eta:=1-\varepsilon$. By Lemma $4.5(\mathrm{a})$, the surfaces $S_{1}, S_{2}$, and $\Phi_{\varepsilon_{i}}^{+}\left(S_{1}, S_{2}\right)$, $i=1, \ldots, 5$, are all pairwise uniformly separated, independent of $S_{1}, S_{2} \in$ $\widetilde{\mathbb{X}}:=\mathbb{X}\left(\Re_{0}, \mu_{1} \Re_{1}\right)$ such that $P S_{1} \leq S_{2}$. Let $\mu_{*} \in(0,1)$ denote the smallest positive value such that

$(4.12 \mathrm{a}, \mathrm{b}) \quad \Phi_{\varepsilon_{5}}^{+}\left(S_{1}, \mu_{*} S_{2}\right) \geq \Phi_{\varepsilon_{4}}^{+}\left(S_{1}, S_{2}\right) ; \quad \Phi_{\varepsilon_{2}}^{+}\left(S_{1}, \mu_{*} S_{2}\right) \geq \Phi_{\varepsilon_{1}}^{+}\left(S_{1}, S_{2}\right)$ 
for all surfaces $S_{1}, S_{2} \in \widetilde{\mathbb{X}}$ such that $P S_{1}<S_{2}$, and let $\mu^{*} \in(1, \infty)$ denote the largest value such that

$$
\Phi_{\varepsilon_{2}}^{+}\left(S_{1}, \mu^{*} S_{2}\right) \leq \Phi_{\varepsilon_{3}}^{+}\left(S_{1}, S_{2}\right)
$$

for all $S_{1}, S_{2} \in \widetilde{\mathbb{X}}$ such that $P S_{1}<S_{2}$. The values $\mu_{*} \in(0,1)$ and $\mu^{*} \in(1, \infty)$ both exist by Lemma 4.4. By (4.12a) and the uniform separation property, there exist constants $0<C_{2} \leq C_{3}$ such that

$$
C_{2} \leq G\left(S_{1}, \mu S_{2} ; x_{0}, x\right) \leq C_{3}
$$

uniformly for all $S_{1}, S_{2} \in \widetilde{\mathbb{X}}$ such that $P S_{1} \leq S_{2}$, all $\mu \in\left[\mu_{*}, \mu^{*}\right]$, all $x_{0}$ such that $\varepsilon_{1} \leq U^{+}\left(S_{1}, S_{2} ; x_{0}\right) \leq \varepsilon_{3}$, and all $x$ such that $U^{+}\left(S_{1}, \mu S_{2} ; x\right)=\varepsilon_{5}$, where $G\left(S_{1}, \mu S_{2} ; x_{0}, x\right)$ denotes Green's function in the domain $\Omega\left(S_{1}, \mu S_{2}\right)$.

For a fixed, but arbitrary pair of surfaces $S_{1}, S_{2} \in \widetilde{\mathbb{X}}$ such that $P S_{1}<S_{2}$ and $S_{2}$ is a $C^{2}$-surface, we define $U(x):=U^{+}\left(S_{1}, S_{2} ; x\right)$ in the closure of $\Omega:=\Omega\left(S_{1}, S_{2}\right)$, and let $U(\mu ; x):=U^{+}\left(S_{1}, \mu S_{2} ; x\right)$ in the closure of $\Omega(\mu):=$ $\Omega\left(S_{1}, \mu S_{2}\right)$ for any $\mu>0$ such that $\mu S_{2}>S_{1}$. For any fixed $\widehat{\mu}>\mu_{*}$, let $z_{0}(\cdot)$ denote a null function such that

$$
\max \left\{U(\mu ; x): x \in(\mu / \widehat{\mu}) S_{1}\right\} \leq \max \left\{U(\widehat{\mu} ; x): x \in(\mu / \widehat{\mu}) S_{1}\right\} \leq z_{0}(\mu-\widehat{\mu})
$$

for $\mu \geq \widehat{\mu}$. It is easily verified that the inequality

$$
0 \leq U(\mu ; x)-U(\widehat{\mu} ;(\widehat{\mu} x / \mu)) \leq z_{0}(\mu-\widehat{\mu})[1-U(\widehat{\mu} ;(\widehat{\mu} x / \mu))]
$$

holds for sufficiently small $\mu>\widehat{\mu}$ and for all $x \in(\mu / \widehat{\mu}) S_{1} \cup \mu S_{2}$. Therefore (4.15) holds for all $x$ in $\Omega\left((\mu / \widehat{\mu}) S_{1}, \mu S_{2}\right)$, by the maximum principle. In view of the assumed smoothness of $S_{2}$ (which implies the uniform continuity of the function $\nabla U(\widehat{\mu} ; x)$ in a neighborhood of $\widehat{\mu} S_{2}$ relative to $\mathrm{Cl}(\Omega(\widehat{\mu}))$; see $[\mathbf{1 4}$, Thm. 8.34]), it follows from (4.15) that

$$
\max \left\{|U(\mu ; x)-U(\widehat{\mu} ;(\widehat{\mu} x / \mu))|: x \in \widehat{\mu} S_{2}\right\} \leq(\mu-\widehat{\mu}) z_{1}(\mu-\widehat{\mu})
$$

as $\mu \downarrow \widehat{\mu}$, where $z_{1}(\cdot)$ denotes a particular null function. We define the function

$$
V(\widehat{\mu}, \mu ; x)=[U(\mu ; x)-U(\widehat{\mu} ; x)] /(\mu-\widehat{\mu})
$$

in $\mathrm{Cl}(\Omega(\widehat{\mu}))$ for $\mu>\widehat{\mu}$. Then for all $x \in \widehat{\mu} S_{2}$, we have

$$
\begin{aligned}
\lim _{\mu \downarrow \widehat{\mu}} V(\widehat{\mu}, \mu ; x) & =\lim _{\mu \downarrow \widehat{\mu}}([U(\widehat{\mu} ;(\widehat{\mu} x / \mu))-U(\widehat{\mu} ; x)] /(\mu-\widehat{\mu})) \\
& =-([x \cdot \nabla U(\widehat{\mu} ; x)] / \widehat{\mu}),
\end{aligned}
$$

where the first equation follows from (4.16) and the second equation can be directly verified by using the known regularity of the function $U(\widehat{\mu} ; \cdot)$. Observe that the convergence in (4.17) is uniform over all $x \in \widehat{\mu} S_{2}$. Obviously, we also have that $V(\widehat{\mu}, \mu ; x)=0$ for $x \in S_{1}$ and $\Delta V(\widehat{\mu}, \mu ; x)=0$ in $\Omega(\widehat{\mu})$, both for any $\mu>\widehat{\mu}$. It follows by the maximum principle that

$$
\max \{|V(\widehat{\mu}, \mu ; x)-W(\widehat{\mu} ; x)|: x \in \operatorname{Cl}(\Omega(\widehat{\mu}))\} \rightarrow 0
$$


as $\mu \downarrow \widehat{\mu}$, where $W(\widehat{\mu} ; x)$ denotes the unique solution of the boundary value problem:

$$
\Delta W(\widehat{\mu} ; x)=0 \quad \text { in } \Omega(\widehat{\mu}),
$$

(4.20) $W\left(\widehat{\mu} ; S_{1}\right)=0 ; \quad W(\widehat{\mu} ; x)=-([x \cdot \nabla U(\widehat{\mu} ; x)] / \widehat{\mu})$ for all $x \in \widehat{\mu} S_{2}$.

By Green's second identity, we have

$$
W\left(\widehat{\mu} ; x_{0}\right)=\int_{\widehat{\mu} S_{2}}\left|\nabla_{x} G\left(\widehat{\mu} ; x_{0}, x\right)\right| W(\widehat{\mu} ; x) d \sigma
$$

for all $x_{0} \in \Omega(\widehat{\mu})$, where $G\left(\widehat{\mu} ; x_{0}, x\right):=G\left(S_{1}, \widehat{\mu} S_{2} ; x_{0}, x\right)$. It follows from (4.14) that

$$
\left(6 C_{2} / \eta\right)(1-U(\widehat{\mu} ; x)) \leq G\left(\widehat{\mu} ; x_{0}, x\right) \leq\left(6 C_{3} / \eta\right)(1-U(\widehat{\mu} ; x))
$$

if $\varepsilon_{1} \leq U\left(x_{0}\right) \leq \varepsilon_{3}$, and if either $U(\widehat{\mu} ; x)=\varepsilon_{5}$ or $U(\widehat{\mu} ; x)=1$. Therefore (4.22) holds whenever $\varepsilon_{1} \leq U\left(x_{0}\right) \leq \varepsilon_{3}$ and $\varepsilon_{5} \leq U(\widehat{\mu} ; x) \leq 1$, by the maximum principle for harmonic functions. Therefore, we have

$$
\left(6 C_{2} / \eta\right)|\nabla U(\widehat{\mu} ; x)| \leq\left|\nabla_{x} G\left(\widehat{\mu} ; x_{0}, x\right)\right| \leq\left(6 C_{3} / \eta\right)|\nabla U(\widehat{\mu} ; x)|
$$

for all $x \in \widehat{\mu} S_{2}$, and for all $x_{0} \in \Omega$ such that $\varepsilon_{1} \leq U\left(x_{0}\right) \leq \varepsilon_{3}$. Since $W(\widehat{\mu} ; x)<0$ throughout $\mathrm{Cl}(\Omega(\widehat{\mu}))$, it follows from (4.20), (4.21), and (4.23) that

$$
6 C_{2} \int_{\widehat{\mu} S_{2}}|\nabla U(\widehat{\mu} ; x)||W(\widehat{\mu} ; x)| d \sigma \leq \eta\left|W\left(\widehat{\mu} ; x_{0}\right)\right|
$$

$$
\leq 6 C_{3} \int_{\widehat{\mu} S_{2}}|\nabla U(\widehat{\mu} ; x)||W(\widehat{\mu} ; x)| d \sigma
$$

provided that $\varepsilon_{1} \leq U\left(x_{0}\right) \leq \varepsilon_{3}$. In view of Lemma 4.6 and the definition of the function $W(\widehat{\mu} ; x)$, it follows from (4.24) that

$$
\left(6 m C_{2} / \eta\right) \leq \widehat{\mu}\left|W\left(\widehat{\mu} ; x_{0}\right)\right| \leq\left(6 M C_{3} / \eta\right)
$$

for $\varepsilon_{1} \leq U\left(x_{0}\right) \leq \varepsilon_{3}$, where $m$ and $M$ denote the constants in Lemma 4.6 in the case where $\Re_{1}$ is replaced by $\mu_{1} \Re_{1}$. Now for any fixed $x_{0} \in \Omega$ with $\varepsilon_{1} \leq U\left(x_{0}\right) \leq \varepsilon_{3}$, the function $\phi(\mu):=U\left(\mu ; x_{0}\right)$ (defined for all $\mu>0$ such that $\left.x_{0} \in \Omega(\mu)\right)$ is clearly continuous. It follows from (4.18) and (4.25) that

$$
\left(6 m C_{2} / \widehat{\mu} \eta\right) \leq-D_{\mu} \phi(\widehat{\mu}):=-\left.D_{\mu} U\left(\mu ; x_{0}\right)\right|_{\mu=\widehat{\mu}} \leq\left(6 M C_{3} / \widehat{\mu} \eta\right),
$$

where $D_{\mu}$ denotes either of the two right-handed Dini derivatives with respect to the variable $\mu$. It follows from (4.26) via a well-known estimate involving Dini derivatives of continuous functions that for any $\alpha, \beta \in\left[\mu_{*}, \mu^{*}\right]$ with $\alpha<\beta$, we have

$$
\begin{aligned}
& \left(6 m C_{2} / \mu^{*} \eta\right)(\beta-\alpha) \leq \phi(\alpha)-\phi(\beta) \\
& =U\left(\alpha ; x_{0}\right)-U\left(\beta ; x_{0}\right) \leq\left(6 M C_{3} / \mu_{*} \eta\right)(\beta-\alpha)
\end{aligned}
$$


for any $x_{0} \in \Omega$ with $\varepsilon_{1} \leq U\left(x_{0}\right) \leq \varepsilon_{3}$. In view of (4.12b), (4.13), it follows from (4.27) that

$$
\left(6 m C_{2} / \mu^{*} \eta \varepsilon_{2}\right)(\beta-\alpha) U(\alpha ; x) \leq U(\alpha ; x)-U(\beta ; x)
$$

for all $\alpha, \beta \in\left[\mu_{*}, \mu^{*}\right]$ with $\alpha<\beta$, and all $x \in \mathrm{Cl}(\Omega(\alpha))$ such that $U(\alpha ; x)=0$ or $U(\alpha ; x)=\varepsilon_{2}$, and that

$$
U(\alpha ; x)-U(\beta ; x) \leq\left(6 M C_{3} / \mu_{*} \eta \varepsilon_{2}\right)(\beta-\alpha) U(\beta ; x)
$$

for all $\alpha, \beta \in\left[\mu_{*}, \mu^{*}\right]$ with $\alpha<\beta$, and all $x \in \mathrm{Cl}(\Omega(\beta))$ such that $U(\beta ; x)=0$ or $U(\beta ; x)=\varepsilon_{2}$. It follows by the maximum principle that (4.28) holds uniformly in $\left\{0 \leq U(\alpha ; x) \leq \varepsilon_{2}\right\}$, and that (4.29) holds uniformly in $\{0 \leq$ $\left.U(\beta ; x) \leq \varepsilon_{2}\right\}$. Since the surfaces $S_{1} \in \widetilde{\mathbb{X}}$ and $S_{2} \in \widetilde{\mathbb{X}} \cap C^{2}$ used in the definition of the functions $U(\mu ; x)$ were arbitrary apart for the requirement that $P S_{1}<S_{2}$, it follows from (4.28) and (4.29) that

$$
\begin{aligned}
C_{0} U^{+}\left(S_{1}, \alpha S_{2} ; x\right) & \leq\left(\left[U^{+}\left(S_{1}, \alpha S_{2} ; x\right)-U^{+}\left(S_{1}, \beta S_{2} ; x\right)\right] /[\beta-\alpha]\right) \\
& \leq C_{1} U^{+}\left(S_{1}, \beta S_{2} ; x\right)
\end{aligned}
$$

for any $S_{1} \in \widetilde{\mathbb{X}}, S_{2} \in \widetilde{\mathbb{X}} \cap C^{2}$ and $\alpha, \beta \in\left[\mu_{*}, \mu^{*}\right]$ such that $\alpha<\beta$, $P S_{1}<S_{2}$ and $P S_{1}<\alpha S_{2}$, and for any $x \in \Omega\left(S_{1}, \Phi_{\varepsilon_{2}}^{+}\left(\alpha S_{2}\right)\right)$, where $C_{0}=$ $\left(6 m C_{2} / \mu^{*} \eta \varepsilon_{2}\right)$ and $C_{1}=\left(6 M C_{3} / \mu_{*} \eta \varepsilon_{2}\right)$. In (4.30), the assumption that $S_{2} \in C^{2}$ is easily eliminated by invoking the continuous dependence of the function $U^{+}\left(S_{1}, \mu S_{2} ; x\right)$ on $S_{2} \in \widetilde{\mathbb{X}}$ (such that $\left.P S_{1}<\mu S_{2}\right)$. Therefore

$$
C_{0} \leq-D_{\mu} \ln \left(U^{+}\left(S_{1}, \mu S_{2} ; x\right)\right) \leq C_{1}
$$

for any $S_{1}, S_{2} \in \widetilde{\mathbb{X}}$ and $\mu \in\left[\mu_{*}, \mu^{*}\right]$ such that $P S_{1}<S_{2}$ and $P S_{1}<\mu S_{2}$, and for any $x \in \Omega\left(S_{1}, \Phi_{\varepsilon_{2}}^{+}\left(\mu S_{2}\right)\right)$, where $D_{\mu}$ denotes any one of the four Dini derivatives. For the particular case where $\mu_{0}=\mu_{*}$ and $\mu_{1}=\mu^{*}$, the assertion follows by integrating (4.31). The original assertion now follows by multiple application of this result.

4.8. Proof of Theorem 4.3. We will prove only (4.6), since the proof of (4.7) is analogous. It is easily seen by a barrier argument that there exist constants $0<C_{2} \leq C_{3}$ such that $\left(1 / C_{3}\right) \leq\left|\nabla U^{+}(\mu ; x)\right| \leq\left(1 / C_{2}\right)$ for $\mu \in\left[\mu_{0}, \mu_{1}\right]$ and $x \in S$. Thus

$$
C_{2} \leq Q^{+}(\mu ; x) \leq C_{3}
$$

for $\mu \in\left[\mu_{0}, \mu_{1}\right]$ and $x \in S$. Choose $\mu_{*} \in\left[\mu_{0}, 1\right)$ such that $\mu_{*} S^{+}>P_{+} S$. Since $S^{+}(\mu)=\mu S^{+}$for $\mu \in\left[\mu_{*}, \mu_{1}\right]$, it follows from Corollary 4.2 (Eq. (4.3)) that there exist constants $0<C_{0} \leq C_{1}$ (the same ones appearing in Eqs. (4.1)-(4.4)) such that

$$
\exp \left(C_{0}(\mu-1)\right) \leq\left(Q^{+}(\mu ; x) / Q^{+}(x)\right) \leq \exp \left(C_{1}(\mu-1)\right)
$$

for $\mu \in\left[1, \mu_{1}\right]$, and

$$
\exp \left(C_{0}(1-\mu)\right) \leq\left(Q^{+}(x) / Q^{+}(\mu ; x)\right) \leq \exp \left(C_{1}(1-\mu)\right)
$$


for $\mu \in\left[\mu_{*}, 1\right]$. For $\mu \in\left[\mu_{0}, \mu_{*}\right]$, we have

$$
S^{+}(\mu) \leq S^{+}\left(\mu_{*}\right) \leq\left(\mu_{*} / \mu\right) S^{+}(\mu),
$$

from which in follows by maximum principles that

$$
0<V^{+}(\mu ; x):=U^{+}\left(S,\left(\mu_{*} / \mu\right) S^{+}(\mu) ; x\right) \leq U^{+}\left(\mu_{*} ; x\right) \leq U^{+}(\mu ; x)
$$

in $\Omega^{+}(\mu)$, and therefore that

$$
\left|\nabla V^{+}(\mu ; x)\right| \leq\left|\nabla U^{+}\left(\mu_{*} ; x\right)\right| \leq\left|\nabla U^{+}(\mu ; x)\right|
$$

for all $x \in S$. Since $S^{+}(\mu) \in \mathbb{X}\left(\Re_{0}, \Re_{1}\right)$, it follows from (4.35), and from (4.3) in the case where $S_{1}:=S, S_{2}:=S^{+}(\mu), \alpha=1$, and $\beta=\left(\mu_{*} / \mu\right)$, that

$$
\begin{aligned}
1 & \leq\left(Q^{+}\left(\mu_{*} ; x\right) / Q^{+}(\mu ; x)\right) \leq\left(\left|\nabla U^{+}(\mu ; x)\right| /\left|\nabla V^{+}(\mu ; x)\right|\right) \\
& \leq \exp \left(\left(C_{1} / \mu_{0}\right)\left(\mu_{*}-\mu\right)\right)
\end{aligned}
$$

for $x \in S$ and $\mu \in\left[\mu_{0}, \mu_{*}\right]$. We have

$$
\exp \left(C_{5}(\mu-1)\right) \leq\left(Q^{+}(\mu ; x) / Q^{+}(x)\right) \leq \exp \left(C_{4}(\mu-1)\right)
$$

for $C_{4}=\left(\left(1-\mu_{*}\right) C_{0} /\left(1-\mu_{0}\right)\right) \in\left(0, C_{0}\right], C_{5}=\left(C_{1} / \mu_{0}\right)>C_{1}, \mu \in\left[\mu_{0}, 1\right]$, and $x \in S$. In fact (4.37) follows from (4.34) if $\mu \in\left[\mu_{*}, 1\right]$. For the case where $\mu \in\left[\mu_{0}, \mu_{*}\right]$, (4.37) follows from (4.36) and (4.34), where we set $\mu=\mu_{*}$ in (4.34). It follows directly from (4.33) that

$$
\exp \left(C_{4}(\mu-1)\right) \leq\left(Q^{+}(\mu ; x) / Q^{+}(x)\right) \leq \exp \left(C_{5}(\mu-1)\right)
$$

for $x \in S$ and $\mu \in\left[1, \mu_{1}\right]$. It then follows from (4.32), and from the theorem of the mean applied to the equivalent logarithmic forms of (4.37) and (4.38), that

$$
\left(C_{2} C_{4}\right)(\mu-1) \leq Q^{+}(\mu ; x)-Q^{+}(x) \leq\left(C_{3} C_{5}\right)(\mu-1)
$$

for $x \in S$, and $\mu \in\left[1, \mu_{1}\right]$, and

$$
\left(C_{3} C_{5}\right)(\mu-1) \leq Q^{+}(\mu ; x)-Q^{+}(x) \leq\left(C_{2} C_{4}\right)(\mu-1)
$$

for $x \in S$ and $\mu \in\left[\mu_{0}, 1\right]$. The assertion (4.6) (with $A_{0}=\left(\mu_{0} C_{2} C_{4}\right)$ and $\left.A_{1}=\left(\mu_{1} C_{3} C_{5}\right)\right)$ follows from (4.39) and (4.40), in view of the fact that

$$
(\mu-1)\left(\mu-1-\mu_{1} \ln (\mu)\right) \leq 0 \leq(\mu-1)\left(\mu-1-\mu_{0} \ln (\mu)\right)
$$

for all $\mu \in\left[\mu_{0}, \mu_{1}\right]$.

\section{A uniform separation estimate.}

Theorem 5.1. Let the values $0<\Re_{0} \leq \Re_{1}$ and $\kappa>0$ be given. For any values $\delta, \delta^{ \pm} \in(0, \kappa]$, let $\mathfrak{Y}\left(\delta^{-}, \delta, \delta^{+}\right)$denote the family of all ordered 4-tuples $\boldsymbol{S}=\left(S_{0}^{-}, S^{-}, S^{+}, S_{0}^{+}\right) \in \mathbf{X}_{4}\left(\Re_{0}, \Re_{1}\right)$ such that $d\left(S^{-}, S^{+}\right) \geq \delta$ and $d\left(S^{ \pm}, S_{0}^{ \pm}\right) \geq \delta^{ \pm}$. Let $F^{ \pm}(x, p, q): \mathbb{R}^{N} \times \mathbb{R}_{+} \times \mathbb{R}_{+} \rightarrow \mathbb{R}$ denote given functions satisfying Assumptions (A1)-(A3). For any $\varepsilon \in(0,1), \delta, \delta^{ \pm} \in(0, \kappa]$, and $\boldsymbol{S}=$ $\left(S_{0}^{-}, S^{-}, S^{+}, S_{0}^{+}\right) \in \mathfrak{Y}\left(\delta^{-}, \delta, \delta^{+}\right)$, let the surfaces $T_{\varepsilon}^{-}\left(S_{0}^{-}, S^{-}, S^{+}\right) \in \mathbb{X}:=$ 
$\mathbb{X}\left(\Re_{0}, \Re_{1}\right)$ and $T_{\varepsilon}^{+}\left(S^{-}, S^{+}, S_{0}^{+}\right) \in \mathbb{X}$ be as defined in $\S 2.3$, where we set $F:=$ $F^{ \pm}(x, p, q)$. Then there exists a strictly positive function $h_{0}\left(t, \varepsilon_{0}, \varepsilon_{1}\right):(0, \kappa] \times$ $(0,1) \times(0,1) \rightarrow \mathbb{R}_{+}$such that for any given values $\varepsilon_{0}, \varepsilon_{1}$ with $0<\varepsilon_{0}<\varepsilon_{1}<$ 1 , we have

$$
d\left(T_{\varepsilon}^{-}\left(S_{0}^{-}, S^{-}, S^{+}\right), T_{\varepsilon}^{+}\left(S^{-}, S^{+}, S_{0}^{+}\right)\right) \geq \delta
$$

for all $\varepsilon \in\left[\varepsilon_{0}, \varepsilon_{1}\right]$, all values $\delta, \delta^{ \pm} \in(0, \kappa]$ such that either $\delta \in\left(0, h_{0}\left(\delta^{+}, \varepsilon_{0}\right.\right.$, $\left.\left.\varepsilon_{1}\right)\right]$ or $\delta \in\left(0, h_{0}\left(\delta^{-}, \varepsilon_{0}, \varepsilon_{1}\right)\right]$, and all ordered 4-tuples $\boldsymbol{S}=\left(S_{0}^{-}, S^{-}, S^{+}, S_{0}^{+}\right) \in$ $\mathfrak{Y}\left(\delta^{-}, \delta, \delta^{+}\right)$.

Lemma 5.2. For given values $0<\Re_{0} \leq \Re_{1}$ and $\delta>0$, let $\boldsymbol{S}=\left(S_{0}^{-}, S^{-}, S^{+}\right.$, $\left.S_{0}^{+}\right) \in \mathbf{X}_{4}\left(\Re_{0}, \Re_{1}\right)$ be such that $d\left(S^{-}, S^{+}\right) \geq \delta$. For fixed $\varepsilon \in(0,1)$, and for all $\sigma^{ \pm} \in \mathbb{X}:=\mathbb{X}\left(\Re_{0}, \Re_{1}\right)$ such that $S^{-} \leq \sigma^{-}<\sigma^{+} \leq S^{+}$, let $t_{\varepsilon}^{-}\left(\sigma^{-}\right):=$ $T_{\varepsilon}^{-}\left(S_{0}^{-}, \sigma^{-}, S^{+}\right) \in \mathbb{X}$ and $t_{\varepsilon}^{+}\left(\sigma^{+}\right):=T_{\varepsilon}^{+}\left(S^{-}, \sigma^{+}, S_{0}^{+}\right) \in \mathbb{X}$, where the operators $T_{\varepsilon}^{ \pm}$are defined as in $\S 2.3$, in terms of given functions $F^{ \pm}(x, p, q)$ satisfying Assumptions (A1)-(A3). Assume that $d\left(\sigma^{\mp}, t_{\varepsilon}^{ \pm}\left(\sigma^{ \pm}\right)\right) \geq \lambda A^{ \pm}$for all $\lambda \in(0, \delta]$ and all surfaces $\sigma^{ \pm} \in \mathbb{X}$ such that $S^{-} \leq \sigma^{-}<\sigma^{+} \leq S^{+}$and $d\left(\sigma^{-}, \sigma^{+}\right) \geq \lambda$, where the values $A^{ \pm}>0$ are fixed. Then

$$
d\left(T_{\varepsilon}^{-}\left(S_{0}^{-}, S^{-}, S^{+}\right), T_{\varepsilon}^{+}\left(S^{-}, S^{+}, S_{0}^{+}\right)\right)=d\left(t_{\varepsilon}^{-}\left(S^{-}\right), t_{\varepsilon}^{+}\left(S^{+}\right)\right) \geq C \delta,
$$

where $C=\min \left\{1, A^{-}, A^{+}\right\} \max \left\{A^{-}, A^{+}\right\}$.

Proof. We will prove the assertion only in the case where $A^{-} \geq A^{+}$, since the opposite case is analogous. Since $d\left(S^{-}, S^{+}\right) \geq \delta$, we have that $d\left(S^{-}, t_{\varepsilon}^{+}\left(S^{+}\right)\right) \geq A^{+} \delta \geq \lambda:=\delta \min \left\{1, A^{+}\right\}$by assumption. In view of this, we have

$$
t_{\varepsilon}^{+}\left(S^{+}\right) \geq S_{\lambda}^{+}:=\partial N_{\lambda}\left(D\left(S^{-}\right)\right) \in \mathbb{X}(\lambda):=\mathbb{X}\left(\Re_{0}+\lambda, \Re_{1}+\lambda\right),
$$

where $S_{\lambda}^{+} \leq S^{+}$because $\lambda \leq \delta$. Let $S_{\lambda, \lambda}^{-}:=\partial N_{\lambda}\left(E\left(S_{\lambda}^{+}\right)\right) \in \mathbb{X}$. Then $S_{\lambda, \lambda}^{-} \geq$ $S^{-}$, since $d\left(x, S_{\lambda}^{+}\right) \geq d\left(S^{-}, S_{\lambda}^{+}\right)=\lambda$ for all $x \in S^{-}$. Since $d\left(S_{\lambda}^{+}, S_{\lambda, \lambda}^{-}\right) \geq \lambda$, we have that $d\left(S_{\lambda}^{+}, t_{\varepsilon}^{-}\left(S_{\lambda, \lambda}^{-}\right)\right) \geq \mu:=A^{-} \lambda$ by assumption. In view of this, we have

$$
t_{\varepsilon}^{-}\left(S^{-}\right) \leq t_{\varepsilon}^{-}\left(S_{\lambda, \lambda}^{-}\right) \leq S_{\lambda, \mu}^{-}:=\partial N_{\mu}\left(E\left(S_{\lambda}^{+}\right)\right) \in \mathbb{X}(\lambda-\mu)
$$

by Lemma 2.5(a). It follows from (5.3) and (5.4) that

$$
d\left(t_{\varepsilon}^{-}\left(S^{-}\right), t_{\varepsilon}^{+}\left(S^{+}\right)\right) \geq d\left(S_{\lambda, \mu}^{-}, S_{\lambda}^{+}\right) \geq \mu:=A^{-} \lambda=A^{-} \min \left\{1, A^{+}\right\} \delta,
$$

which is (5.2) in the case $0<A^{+} \leq A^{-}$.

Lemma 5.3. Let $K=K\left(\rho, r_{0}, \nu_{0}\right)$ denote the convex hull of the set $\{0\} \cup$ $\bar{B}\left(r_{0} \nu_{0} ; \rho\right)$, where the constants $0<\rho<r_{0}$ and the unit vector $\nu_{0}$ are fixed. Let $S_{0}=\partial K$, and let $S_{\delta}=\partial N_{\delta}(K)$ for any $\delta>0$. Given a (large) positive 
constant $\kappa$, let $\phi(\varepsilon):=\min \left\{(|x| / 2 \kappa): x \in \Phi_{\varepsilon}^{+}\left(S_{0}, S_{2 \kappa}\right)\right\}$ for each $\varepsilon \in[0,1]$. Then, in the context of $\S 2.3$, with $\Re_{0}=0$, we have

$$
T_{\varepsilon}\left(S_{0}, S_{\delta}, S_{\alpha}\right) \geq \partial B(0 ; \lambda \delta)
$$

for any $\delta, \delta_{0} \in(0, \kappa]$ and $0<\varepsilon \leq \varepsilon_{1}<1$, where $\alpha:=\delta+\delta_{0}$ and we set $\lambda=$ $\phi\left(1-\varepsilon_{1}\right)$. Also, there exists a strictly-positive function $h\left(\lambda, \delta_{0}, \varepsilon_{0}\right):[1, \infty) \times$ $(0, \kappa] \times(0,1) \rightarrow \mathbb{R}_{+}$such that $h\left(\lambda, \delta_{0}, \varepsilon_{0}\right)<\delta_{0}$, and such that (5.5) holds whenever $\delta_{0} \in(0, \kappa], \lambda \geq 1,0<\varepsilon_{0} \leq \varepsilon<1$, and $\delta \in\left(0, h\left(\lambda, \delta_{0}, \varepsilon_{0}\right)\right]$.

Proof. We have that $\mu S_{t} \leq S_{\mu t}$ for any $t \geq 0$ and $0<\mu \leq 1$. In view of Lemma 2.4(a),(b), it follows that

$$
\begin{aligned}
\Phi_{\varepsilon}^{-}\left(S_{0}, S_{\delta}\right) & \geq \Phi_{\varepsilon}^{-}\left((\delta / \eta) S_{0},(\delta / \eta) S_{\eta}\right) \\
& =(\delta / \eta) \Phi_{\varepsilon}^{-}\left(S_{0}, S_{\eta}\right) \geq \partial B(0 ; \delta \phi(1-\varepsilon)), \\
\Phi_{\varepsilon}^{+}\left(S_{\delta}, S_{\alpha}\right) & \geq \Phi_{\varepsilon}^{+}\left(S_{0}, S_{\alpha}\right) \geq \Phi_{\varepsilon}^{+}\left((\alpha / \eta) S_{0},(\alpha / \eta) S_{\eta}\right) \\
& =(\alpha / \eta) \Phi_{\varepsilon}^{+}\left(S_{0}, S_{\eta}\right) \geq \partial B(0 ; \alpha \phi(\varepsilon))
\end{aligned}
$$

for any $\varepsilon \in(0,1)$ and $0<\delta<\alpha \leq \eta:=2 \kappa$. Obviously

(5.8) $T_{\varepsilon}\left(S_{0}, S_{\delta}, S_{\alpha}\right) \geq \Phi_{\varepsilon}^{-}\left(S_{0}, S_{\delta}\right) \geq \partial B(0 ; \delta \phi(1-\varepsilon)) \geq \partial B\left(0 ; \delta \phi\left(1-\varepsilon_{1}\right)\right)$

for any $0<\delta<\alpha \leq \eta$ and $0<\varepsilon \leq \varepsilon_{1}<1$, due to (5.6). By (5.6), (5.7), and Lemmas 2.5(a) and 3.4, we have

$$
\begin{aligned}
T_{\varepsilon}\left(S_{0}, S_{\delta}, S_{\alpha}\right) & \geq \Psi_{\varepsilon}(\partial B(0 ; \delta \phi(1-\varepsilon)), \partial B(0 ; \alpha \phi(\varepsilon))) \\
& \geq \partial B(0 ;([\alpha \phi(\varepsilon)+\delta A \phi(1-\varepsilon)] /[1+A])) \\
& \geq \partial B\left(0 ;\left[\delta_{0} \phi\left(\varepsilon_{0}\right) /(1+A)\right]\right)
\end{aligned}
$$

for any $0<\delta<\alpha \leq \eta$ and $0<\varepsilon_{0} \leq \varepsilon<1$, where $A=A([\alpha \phi(\varepsilon)-\delta \phi(1-$ $\varepsilon)] / \varepsilon)$. If we assume that $\delta_{0} \in(0, \kappa]$ and $0<\delta<\delta_{0} \min \left\{1,\left[\phi\left(\varepsilon_{0}\right) / 2 \phi(1-\right.\right.$ $\left.\left.\left.\varepsilon_{0}\right)\right]\right\}$, then we have

$$
\left(\delta_{0} \phi\left(\varepsilon_{0}\right) / 2\right) \leq([\alpha \phi(\varepsilon)-\delta \phi(1-\varepsilon)] / \varepsilon) \leq\left(\eta / \varepsilon_{0}\right),
$$

from which it follows that $A=A\left(\left[\delta_{0} \phi\left(\varepsilon_{0}\right) / 2\right],\left[\eta / \varepsilon_{0}\right]\right)$ in (5.9). The assertion (5.5) now follows easily from (5.8), (5.9), and (5.10).

5.4. Proof of Theorem 5.1. For any point $x_{0} \in B\left(0 ; \Re_{1}\right) \backslash B\left(0 ; \Re_{0}\right)$, we define $K^{ \pm}\left(x_{0}\right)$ to be the convex hull of the set $\left\{x_{0}\right\} \cup \bar{B}\left(\left(1 \pm\left(r_{0} /\left|x_{0}\right|\right)\right) x_{0} ; \rho\right)$, where the constants $0<\rho<r_{0}$ are fixed such that $r_{0} \leq \Re_{0}$ and $\left(\rho / r_{0}\right) \leq$ $\left(\Re_{0} / \Re_{1}\right)$. We set $S^{ \pm}\left(x_{0}\right):=\partial K^{ \pm}\left(x_{0}\right)$ and $S^{ \pm}\left(x_{0} ; \delta\right):=\partial N_{\delta}\left(K^{ \pm}\left(x_{0}\right)\right)$ for $\delta>$ 0 . Let $F_{0}^{ \pm}(p, q): \mathbb{R}_{+}^{2} \rightarrow \mathbb{R}$ denote fixed, continuously-differentiable functions satisfying Assumptions (A1)-(A3), and such that $\pm\left(F_{0}^{ \pm}(p, q)-F^{ \pm}(x, p, q)\right) \geq$ 0 for all $x \in B\left(0 ; 2 \Re_{1}\right)$ and all $p, q>0$. For any $\varepsilon \in(0,1), \delta, \delta^{ \pm} \in(0, \kappa]$, 4-tuple $\boldsymbol{S}=\left(S_{0}^{-}, S^{-}, S^{+}, S_{0}^{+}\right) \in \mathfrak{Y}\left(\delta^{-}, \delta, \delta^{+}\right)$, and $x_{0} \in S^{-}$, let the surfaces $T_{0, \varepsilon}^{+}\left(S^{-}, S^{+}, S_{0}^{+}\right) \in \mathbb{X}$ and $T_{0, \varepsilon}^{+}\left(S^{-}\left(x_{0} ; 0\right), S^{-}\left(x_{0} ; \delta\right), S^{-}\left(x_{0} ; \delta+\delta^{+}\right)\right) \in \mathfrak{X}$ be defined by Eqs. (2.3), (2.4), and (2.5), where we choose $F(x, p, q):=$ 
$F_{0}^{+}(p, q)$. (The latter surface is in $\mathfrak{X}$ because it is starlike relative to a sufficiently small ball centered at $x_{0}$.) Then

$$
S^{-}\left(x_{0}\right) \leq S^{-}, S^{-}\left(x_{0} ; \delta\right) \leq S^{+}, S^{-}\left(x_{0} ; \delta+\delta^{+}\right) \leq S_{0}^{+},
$$

from which it follows by Lemma 2.5(a) (in a slightly generalized context admitting the same proof) and Lemma 5.3 that for any given $\varepsilon_{0}, \varepsilon_{1} \in(0,1)$, we have

$$
\begin{aligned}
T_{\varepsilon}^{+}\left(S^{-}, S^{+}, S_{0}^{+}\right) & \geq T_{0, \varepsilon}^{+}\left(S^{-}, S^{+}, S_{0}^{+}\right) \\
& \geq T_{0, \varepsilon}^{+}\left(S^{-}\left(x_{0}\right), S^{-}\left(x_{0} ; \delta\right), S^{-}\left(x_{0} ; \delta+\delta^{+}\right)\right) \\
& \geq \partial B\left(x_{0} ; \lambda \delta\right)
\end{aligned}
$$

if $\varepsilon \in\left(0, \varepsilon_{1}\right], \lambda=\phi\left(1-\varepsilon_{1}\right)$, and $x_{0} \in S^{-}$, or if $\varepsilon \in\left[\varepsilon_{0}, 1\right), \lambda \geq 1, \delta \in$ $\left(0, h\left(\lambda, \delta^{+}, \varepsilon_{0}\right)\right]$, and $x_{0} \in S^{-}$. Since $x_{0}$ is arbitrary in $S^{-}$, it follows from (5.11) that

$$
d\left(S^{-}, T_{\varepsilon}^{+}\left(S^{-}, S^{+}, S_{0}^{+}\right)\right) \geq \lambda \delta
$$

if $\varepsilon \in\left(0, \varepsilon_{1}\right]$ and $\lambda=\phi\left(1-\varepsilon_{1}\right)$, or if $\varepsilon \in\left[\varepsilon_{0}, 1\right), \lambda \geq 1$, and $\delta \in\left(0, h\left(\lambda, \delta^{+}, \varepsilon_{0}\right)\right]$. Only for the remainder of this proof, we extend the partial ordering in $\mathbb{X}$ to the family $\mathfrak{X}_{0}:=\{S \in \mathfrak{X}: 0 \notin S\}$ (where 0 denotes the origin) in such a way that $S_{1} \leq S_{2}$ (resp. $\left.S_{1}<S_{2}\right)$ if $G\left(S_{1}\right) \subset G\left(S_{2}\right)\left(\mathrm{Cl}\left(G\left(S_{1}\right)\right) \subset G\left(S_{2}\right)\right)$, where $G\left(S_{i}\right)$ denotes the connected component of the complement of $S_{i}$ (in $\mathbb{R}^{N}$ ) which contains the origin. For any $\delta, \delta^{ \pm} \in(0, \kappa], \varepsilon \in(0,1)$, $\boldsymbol{S}=\left(S_{0}^{-}, S^{-}, S^{+}, S_{0}^{+}\right) \in \mathfrak{Y}\left(\delta^{-}, \delta, \delta^{+}\right)$, and $x_{0} \in S^{+}$, let the surfaces $T_{0, \varepsilon}^{-}\left(S_{0}^{-}, S^{-}, S^{+}\right) \in \mathbb{X}$ and $T_{0, \varepsilon}^{-}\left(S^{+}\left(x_{0} ; \delta+\delta^{-}\right), S^{+}\left(x_{0} ; \delta\right), S^{+}\left(x_{0}\right)\right) \in \mathfrak{X}_{0}$ be defined by Eqs. (2.3), (2.4), and (2.5), where " $<$ " and " $\leq$ " are interpreted in the present sense, and where we set $F(x, p, q)=F_{0}^{-}(p, q)$. Since

$$
S^{+} \leq S^{+}\left(x_{0}\right), S^{-} \leq S^{+}\left(x_{0} ; \delta\right), S_{0}^{-} \leq S^{+}\left(x_{0} ; \delta+\delta^{-}\right)
$$

it follows from Lemma 2.5(a) (in a generalized context permitting the same proof) and Lemma 5.3 that

$$
\begin{aligned}
T_{\varepsilon}^{-}\left(S_{0}^{-}, S^{-}, S^{+}\right) & \leq T_{0, \varepsilon}^{-}\left(S_{0}^{-}, S^{-}, S^{+}\right) \\
& \leq T_{0, \varepsilon}^{-}\left(S^{+}\left(x_{0} ; \delta+\delta^{-}\right), S^{+}\left(x_{0} ; \delta\right), S^{+}\left(x_{0}\right)\right) \\
& \leq \partial B\left(x_{0}, \lambda \delta\right)
\end{aligned}
$$

if $\varepsilon \in\left(0, \varepsilon_{1}\right], \lambda=\phi\left(1-\varepsilon_{1}\right)$, and $x_{0} \in S^{+}$, or if $\varepsilon \in\left[\varepsilon_{0}, 1\right), \lambda \geq 1, \delta \in$ $\left(0, h\left(\lambda, \delta^{-}, \varepsilon_{0}\right)\right]$, and $x_{0} \in S^{+}$. Since $x_{0}$ is arbitrary in $S^{+}$, it follows from (5.13) that

$$
d\left(S^{+}, T_{\varepsilon}^{-}\left(S_{0}^{-}, S^{-}, S^{+}\right)\right) \geq \lambda \delta
$$

if $\varepsilon \in\left(0, \varepsilon_{1}\right]$ and $\lambda=\phi\left(1-\varepsilon_{1}\right)$, or if $\varepsilon \in\left[\varepsilon_{0}, 1\right), \lambda \geq 1$, and $\delta \in\left(0, h\left(\lambda, \delta^{-}, \varepsilon_{0}\right)\right]$. We define the function $h_{0}\left(t, \varepsilon_{0}, \varepsilon_{1}\right):(0, \kappa] \times(0,1) \times(0,1) \rightarrow \mathbb{R}_{+}$such that $h_{0}\left(t, \varepsilon_{0}, \varepsilon_{1}\right):=h\left(\lambda, t, \varepsilon_{0}\right)$ in the particular case where $\lambda=\left(1 / \phi\left(1-\varepsilon_{1}\right)\right)$. At 
this point, the assertion follows from Lemma 5.2 and Eqs. (5.12) and (5.14), where we set $\lambda=\left(1 / \phi\left(1-\varepsilon_{1}\right)\right)$.

\section{Uniform contraction property for uniformly-separated ordered triples.}

Theorem 6.1. In the context of $\S 2.3$, for given values $0<\Re_{0} \leq \Re_{1}, P>$ 1 , and $\varepsilon \in(0,1)$, there exist constants $A_{0} \in(0,1)$ and $B_{0} \in(0,1 / 2)$ such that

$$
T_{\varepsilon}\left(\widehat{S}^{-}, \widehat{S}, \widehat{S}^{+}\right) \leq \lambda^{\left(1-\kappa_{0}\right) r} T_{\varepsilon}\left(S^{-}, S, S^{+}\right),
$$

uniformly for all values $\lambda>1, \lambda^{ \pm} \geq 1$, and $\kappa_{0} \in(0,1)$ such that

$$
\lambda^{\kappa_{0}}\left(\lambda_{-} / \lambda\right)^{A\left(\lambda_{-} / \lambda\right)(1-B)}\left(\lambda_{+} / \lambda\right)^{A\left(\lambda_{+} / \lambda\right) B} \leq 1,
$$

with

$$
B=B\left(\left(\lambda_{+} / \lambda\right)^{A\left(\lambda_{+} / \lambda\right)}\left(\lambda / \lambda_{-}\right)^{A\left(\lambda_{-} / \lambda\right)}\right),
$$

and uniformly for all $r \geq 0$ and all surfaces $S, S^{ \pm}, \widehat{S}, \widehat{S}^{ \pm} \in \mathbb{X}:=\mathbb{X}\left(\Re_{0}, \Re_{1}\right)$ such that $P^{2} S^{-} \leq P S \leq S^{+}, P^{2} \widehat{S}^{-} \leq P \widehat{S} \leq \widehat{S}^{+}$, and

$$
\widehat{S} \leq \lambda^{r} S, \quad \widehat{S}^{ \pm} \leq \lambda_{ \pm}^{r} S^{ \pm} .
$$

Here, we define the functions $A(t), B(t): \mathbb{R}_{+} \rightarrow \mathbb{R}_{+}$such that $A(t)=A_{0}$ and $B(t)=B_{0}$ for $0<t<1$, and $A(t)=1$ and $B(t)=B_{1}:=\left(1-B_{0}\right)$ for $t \geq 1$.

Remark 6.2. It follows from Theorem 6.1 that

$$
\begin{aligned}
& \mu M\left(T_{\varepsilon}\left(S^{-}, S, S^{+}\right), T_{\varepsilon}\left(\widehat{S}^{-}, \widehat{S}, \widehat{S}^{+}\right)\right) \\
& \leq\left(1-\kappa_{0}\right) \max \left\{\mu M(S, \widehat{S}), \mu^{-} M\left(S^{-}, \widehat{S}^{-}\right), \mu^{+} M\left(S^{+}, \widehat{S}^{+}\right)\right\}
\end{aligned}
$$

uniformly for all $\lambda, \lambda^{ \pm}>1$ and $\kappa_{0} \in(0,1)$ satisfying (6.2), (6.3), and all surfaces $S, S^{ \pm}, \widehat{S}, \widehat{S}^{ \pm} \in \mathbb{X}:=\mathbb{X}\left(\Re_{0}, \Re_{1}\right)$ such that $P^{2} S^{-} \leq P S \leq S^{+}$and $P^{2} \widehat{S}^{-} \leq P \widehat{S} \leq \widehat{S}^{+}$. Here, the metric $M$ is defined in (2.2), and we define $\mu=(1 / \ln (\lambda))$ and $\mu^{ \pm}=\left(1 / \ln \left(\lambda_{ \pm}\right)\right)$.

Remark 6.3. The main assumption for Theorem 6.1 (namely Eq. (6.2)) actually requires that $\lambda_{-}^{\alpha} \lambda_{+}^{\beta} \leq \lambda^{\left(1-\widetilde{\kappa}_{0}\right)}$ when $1 \leq \lambda_{-}<\lambda<\lambda_{+}$, and that $\lambda_{-}^{\beta} \lambda_{+}^{\alpha} \leq \lambda^{\left(1-\widetilde{\kappa}_{0}\right)}$ when $1 \leq \lambda_{+}<\lambda<\lambda_{-}$, where $\alpha=\left(A_{0} B_{0}\right) /\left(A_{0} B_{0}+B_{1}\right) \in$ $(0,1 / 2), \beta=(1-\alpha) \in(1 / 2,1)$, and $\widetilde{\kappa}_{0}=\left(\kappa_{0} /\left(A_{0} B_{0}+B_{1}\right)\right)>0$. In the case where $1 \leq \lambda_{-}<\lambda>\lambda_{+} \geq 1$, Eq. (6.2) requires that $\lambda_{-}^{(1-B)} \lambda_{+}^{B} \leq$ $\lambda^{\left(1-\left[\kappa_{0} / A_{0}\right]\right)}$ for $B \in\left\{B_{0}, B_{1}\right\}$. 
Lemma 6.4. In the context of $\S 2.3$, for given values $0<\Re_{0}<\Re_{1}, P>1$, and $\varepsilon \in(0,1)$, there exists a constant $A_{0} \in(0,1)$ such that

$(6.6 \mathrm{a}, \mathrm{b}) \Phi_{\varepsilon}^{-}\left(\lambda S_{1}, S_{2}\right) \leq \lambda^{A_{0}} \Phi_{\varepsilon}^{-}\left(S_{1}, S_{2}\right) ; \quad \Phi_{\varepsilon}^{+}\left(S_{1}, \lambda S_{2}\right) \leq \lambda^{A_{0}} \Phi_{\varepsilon}^{+}\left(S_{1}, S_{2}\right)$

uniformly for all $\lambda \in\left[P^{-1 / 2}, 1\right]$ and for all $S_{1}, S_{2} \in \mathbb{X}:=\mathbb{X}\left(\Re_{0}, \Re_{1}\right)$ such that $P S_{1} \leq S_{2}$.

Proof. For fixed surfaces $S_{1}, S_{2} \in \mathbb{X}$ such that $P S_{1} \leq S_{2}$, and we define $U^{+}(\lambda ; x)=U^{+}\left(S_{1}, \lambda S_{2} ; x\right)$ and $U^{-}(\lambda ; x)=U^{-}\left(\lambda S_{1}, S_{2} ; x\right)$ for any $\lambda \in$ $\left[P^{-1 / 2}, 1\right]$. For a given a unit vector $e$, and for any $\lambda \in\left[P^{-1 / 2}, 1\right]$, choose the values $\alpha^{ \pm}(\lambda)>0$ such that $U^{ \pm}\left(\lambda ; \alpha^{ \pm}(\lambda) e\right)=\varepsilon$. This is equivalent to the requirement that $\alpha^{-}(\lambda) e \in \Phi_{\varepsilon}^{-}\left(\lambda S_{1}, S_{2}\right)$ and $\alpha^{+}(\lambda) e \in \Phi_{\varepsilon}^{+}\left(S_{1}, \lambda S_{2}\right)$ for any $\lambda \in\left[P^{-1 / 2}, 1\right]$. Thus $\alpha^{ \pm}(\lambda) \leq \alpha^{ \pm}(\mu)$ for $P^{-1 / 2} \leq \lambda \leq \mu \leq 1$, by Lemma 2.4(a). By Theorem 4.1 and the theorem of the mean, we have

$$
\begin{aligned}
& \mathfrak{M}_{0}^{ \pm}(\lambda, \mu)\left(\alpha^{ \pm}(\mu)-\alpha^{ \pm}(\lambda)\right) \\
& \geq \pm\left(U^{ \pm}\left(\lambda ; \alpha^{ \pm}(\mu) e\right)-U^{ \pm}\left(\lambda ; \alpha^{ \pm}(\lambda) e\right)\right) \\
& = \pm\left(U^{ \pm}\left(\lambda ; \alpha^{ \pm}(\mu) e\right)-U^{ \pm}\left(\mu ; \alpha^{ \pm}(\mu) e\right)\right) \\
& \geq \pm\left(\exp \left( \pm C_{0}(\mu-\lambda)\right)-1\right) \varepsilon,
\end{aligned}
$$

for any values $P^{-1 / 2} \leq \lambda \leq \mu \leq 1$, where $\mathfrak{M}_{0}^{ \pm}(\lambda, \mu)=\max \left\{\left|e \cdot \nabla U^{ \pm}\left(\lambda ; \gamma^{ \pm} e\right)\right|\right.$ : $\left.\gamma^{ \pm} \in\left[\alpha^{ \pm}(\lambda), \alpha^{ \pm}(\mu)\right]\right\}$. It follows from (6.7) in the limit as $\mu \rightarrow \lambda$ or $\lambda \rightarrow \mu$ that $D_{\lambda} \alpha^{ \pm}(\lambda) \geq\left(C_{0} / \mathfrak{M}_{1}^{ \pm}\right)$for $\lambda \in\left(P^{-1 / 2}, 1\right)$, where $\mathfrak{M}_{1}^{ \pm}=$ $\max \left\{\left|\nabla U^{ \pm}\left(\lambda ; \alpha^{ \pm}(\lambda) e\right)\right|: \lambda \in\left[P^{-1 / 2}, 1\right]\right\}$ and $D_{\lambda}$ denotes any Dini derivative. Thus $\lambda D_{\lambda} \ln \left(\alpha^{ \pm}(\lambda)\right) \geq\left(C_{0} / \Re_{1} P^{1 / 2} \mathfrak{M}_{1}^{ \pm}\right)$for $\lambda \in\left(P^{-1 / 2}, 1\right)$. Since $\alpha^{ \pm}(\lambda)$ depends continuously on $\lambda \in\left[P^{-1 / 2}, 1\right]$, one concludes that $\alpha^{ \pm}(\lambda) \leq$ $\lambda^{a} \alpha^{ \pm}(1)$ for $\lambda \in\left[P^{-1 / 2}, 1\right]$, where $a$ denotes any constant in the interval $\left(0,\left(C_{0} / \mathfrak{R}_{1} P^{1 / 2} \mathfrak{M}_{1}^{ \pm}\right)\right]$. By Lemma $4.5(\mathrm{~b})$, there exist constants $C_{1}^{ \pm}$such that $\mathfrak{M}_{1}^{ \pm}=\mathfrak{M}_{1}^{ \pm}\left(S_{1}, S_{2} ; e\right) \leq C_{1}^{ \pm}$, uniformly over all $S_{1}, S_{2} \in \mathbb{X}$ such that $P S_{1} \leq S_{2}$, and over all $e \in \partial B(0 ; 1)$. This completes the proof of the estimates $(6.6 \mathrm{a}, \mathrm{b})$, where $A_{0}$ denotes any constant in the interval $(0,1 / 2] \mathrm{such}$ that $A_{0} \leq\left(C_{0} / \Re_{1} P^{1 / 2} C_{1}^{ \pm}\right)$.

Lemma 6.5. In the context of $\S 2.3$, for given values $0<\Re_{0}<\Re_{1}, P>1$, and $\varepsilon \in(0,1)$, there exists a constant $A_{0} \in(0,1)$ such that

$$
\begin{aligned}
& \Phi_{\varepsilon}^{+}\left(\widehat{S}_{1}, \widehat{S}_{2}\right) \leq \lambda_{1}^{\left(1-A\left(\lambda_{2} / \lambda_{1}\right)\right)} \lambda_{2}^{A\left(\lambda_{2} / \lambda_{1}\right)} \Phi_{\varepsilon}^{+}\left(S_{1}, S_{2}\right), \\
& \Phi_{\varepsilon}^{-}\left(\widehat{S}_{1}, \widehat{S}_{2}\right) \leq \lambda_{1}^{A\left(\lambda_{1} / \lambda_{2}\right)} \lambda_{2}^{\left(1-A\left(\lambda_{1} / \lambda_{2}\right)\right)} \Phi_{\varepsilon}^{-}\left(S_{1}, S_{2}\right),
\end{aligned}
$$

for any values $\lambda_{1}, \lambda_{2}>0$ and any surfaces $S_{1}, S_{2}, \widehat{S}_{1}, \widehat{S}_{2} \in \mathbb{X}:=\mathbb{X}\left(\Re_{0}, \Re_{1}\right)$ such that $P S_{1} \leq S_{2}, \widehat{S}_{1}<\widehat{S}_{2}, \widehat{S}_{1} \leq \lambda_{1} S_{1}$, and $\widehat{S}_{2} \leq \lambda_{2} S_{2}$, where $A(\lambda)=$ $A_{0} \in(0,1)$ for $\lambda \in(0,1)$ and $A(\lambda)=1$ for $\lambda \in[1, \infty)$. 
Proof. It suffices to prove (6.8) and (6.9) in the case where $\lambda_{1}, \lambda_{2} \in\left[\left(\Re_{0} / \Re_{1}\right)\right.$, $\left(\Re_{1} / \Re_{0}\right)$. In fact, since $\partial B\left(0 ; \Re_{0}\right) \leq \widehat{S}_{i} \leq \lambda_{i} S_{i} \leq \lambda_{i} \partial B\left(0 ; \Re_{1}\right)$ for $i=$ 1,2 by assumption, we must have $\lambda_{1}, \lambda_{2} \geq\left(\Re_{0} / \Re_{1}\right)$ for any admissible configuration. On the other hand, for any $S_{1}, S_{2}, \widehat{S}_{1}, \widehat{S}_{2} \in \mathbb{X}$, there exist values $\lambda_{1}, \lambda_{2} \in\left(0,\left(\Re_{1} / \Re_{0}\right)\right]$ such that $\widehat{S}_{1} \leq \lambda_{1} S_{1}$ and $\widehat{S}_{2} \leq \lambda_{2} S_{2}$, and the assertions in the general case follow directly from this case. We will only prove (6.8), since the proof of (6.9) (based on (6.6a)) is analogous. For the case where $\left(\Re_{0} / \Re_{1}\right)^{2} \leq\left(\lambda_{2} / \lambda_{1}\right)<1$, choose a fixed value $\alpha \in(0,1)$ so small that $\left(\lambda_{2} / \lambda_{1}\right)^{\alpha} \geq\left(\Re_{0} / \Re_{1}\right)^{2 \alpha} \geq P^{-1 / 2}$. Since $\widehat{S}_{1} \leq \lambda_{1} S_{1}$ and $\widehat{S}_{2} \leq \lambda_{2} S_{2} \leq$ $\lambda_{1}\left(\lambda_{2} / \lambda_{1}\right)^{\alpha} S_{2}$, it follows from Lemmas 2.4(a),(b) and 6.4 (Eq. (6.6b)) that

$$
\begin{aligned}
& \Phi_{\varepsilon}^{+}\left(\widehat{S}_{1}, \widehat{S}_{2}\right) \leq \Phi_{\varepsilon}^{+}\left(\lambda_{1} S_{1}, \lambda_{1}\left(\lambda_{2} / \lambda_{1}\right)^{\alpha} S_{2}\right) \\
& =\lambda_{1} \Phi_{\varepsilon}^{+}\left(S_{1},\left(\lambda_{2} / \lambda_{1}\right)^{\alpha} S_{2}\right) \leq \lambda_{1}\left(\lambda_{2} / \lambda_{1}\right)^{\alpha \widehat{A}_{0}} \Phi_{\varepsilon}^{+}\left(S_{1}, S_{2}\right),
\end{aligned}
$$

where $\widehat{A}_{0} \in(0,1)$ denotes the constant in Lemma 6.4. Thus (6.8) holds in the case where $0<\lambda_{2}<\lambda_{1}$, where we have defined $A_{0}=\alpha \widehat{A}_{0}$. For the case where $0<\lambda_{1} \leq \lambda_{2}$, it follows from Lemmas 2.4(a),(b) that

$$
\Phi_{\varepsilon}^{+}\left(\widehat{S}_{1}, \widehat{S}_{2}\right) \leq \Phi_{\varepsilon}^{+}\left(\lambda_{2} S_{1}, \lambda_{2} S_{2}\right)=\lambda_{2} \Phi_{\varepsilon}^{+}\left(S_{1}, S_{2}\right),
$$

which is the assertion in this case.

Lemma 6.6. In the context of $\S 2.3$, for given values $0<\Re_{0} \leq \Re_{1}, P>1$, and $\varepsilon \in(0,1)$, there exists a constant $b \in[1 / 2,1)$ such that

(6.10a, b) $\quad \Psi_{\varepsilon}\left(S_{1}, \lambda S_{2}\right) \leq \lambda^{b} \Psi_{\varepsilon}\left(S_{1}, S_{2}\right) ; \quad \Psi_{\varepsilon}\left(\lambda S_{1}, S_{2}\right) \leq \lambda^{b} \Psi_{\varepsilon}\left(S_{1}, S_{2}\right)$,

both uniformly for all $\lambda \in\left[1, P^{1 / 2}\right]$ and all $S_{1}, S_{2} \in \mathbb{X}:=\mathbb{X}\left(\Re_{0}, \Re_{1}\right)$ such that $P S_{1}<S_{2}$.

Proof. We will prove only (6.10a), since the proof of (6.10b) is analogous. By Lemma 3.6 (Eq. (3.7)), we have

$$
d\left(x, S_{1}\right) \geq \Re_{0}\left(P^{1 / 2}-1\right) ; d\left(y, \lambda S_{2}\right) \geq d\left(y, S_{2}\right) \geq \Re_{0}\left(1-P^{-1 / 2}\right)
$$

for any $\lambda \geq 1$, any $S_{1}, S_{2} \in \mathbb{X}$ such that $P S_{1} \leq S_{2}$, and any $x \in E\left(P^{1 / 2} S_{1}\right)$ and $y \in D\left(P^{-1 / 2} S_{2}\right)$. Also, by Lemma 3.6 (Eq. (3.8)), we have

$$
\begin{aligned}
& \left(\Re_{0} / P^{1 / 2}\right)(\lambda-1) \leq d\left(x, S_{1}\right)-d\left(x, \lambda S_{1}\right) ; \\
& d\left(y, \mu S_{2}\right)-d\left(y, \lambda S_{2}\right) \leq \Re_{1} P^{1 / 2}(\mu-\lambda)
\end{aligned}
$$

for all $S_{1}, S_{2} \in \mathbb{X}, 1 \leq \lambda \leq \mu \leq P^{1 / 2}, x \in E\left(\lambda S_{1}\right)$, and $y \in D\left(\lambda S_{2}\right)$. We have $\Omega\left(S_{1}, \lambda S_{2}\right) \supset D\left(P^{-1 / 2} S_{2}\right) \cap E\left(P^{1 / 2} S_{1}\right)$ for any $\lambda \geq 1$ and any $S_{1}, S_{2} \in \mathbb{X}$ such that $P S_{1} \leq S_{2}$. Therefore, it follows from (6.11) that

$$
d\left(x, S_{1}\right)+d\left(x, \lambda S_{2}\right) \geq \Re_{0}\left(1-P^{-1 / 2}\right)
$$


uniformly for all $\lambda \geq 1$, all $S_{1}, S_{2} \in \mathbb{X}$ such that $P S_{1} \leq S_{2}$, and all $x \in$ $\Omega\left(S_{1}, \lambda S_{2}\right)$. We also have that

$$
\begin{aligned}
d\left(x, S_{1}\right)+d\left(x, \lambda S_{2}\right) & \leq d\left(x, \partial B\left(0 ; \Re_{0}\right)\right)+d\left(x, \partial B\left(0 ; \lambda \Re_{1}\right)\right) \\
& \leq \lambda \Re_{1}-\Re_{0} \leq P^{1 / 2} \Re_{1}
\end{aligned}
$$

uniformly for all $S_{1}, S_{2} \in \mathbb{X}$ such that $S_{1}<S_{2}$, all $\lambda \in\left[1, P^{1 / 2}\right]$, and all $x \in \Omega\left(S_{1}, \lambda S_{2}\right)$. In view of (6.13), (6.14), and Lemma 3.4, there exist values $0<a_{0}<a_{1}$ such that

$$
a_{0} \leq d\left(x, S_{1}\right) \leq a_{1} ; \quad a_{0} \leq d\left(x, \lambda S_{2}\right) \leq a_{1},
$$

both uniformly for all $\lambda \in\left[1, P^{1 / 2}\right]$, all $S_{1}, S_{2} \in \mathbb{X}$ such that $P S_{1} \leq S_{2}$, and all $x \in \Omega\left(S_{1}, \lambda S_{2}\right)$ such that $f_{\varepsilon}\left(x, d\left(x, S_{1}\right), d\left(x, \lambda S_{2}\right)\right)=0$, where we define $f_{\varepsilon}(x, p, q)=f(x, p / \varepsilon, q / \varepsilon)=F(x, \varepsilon / p, \varepsilon / q)$. For fixed admissible $S_{1}, S_{2}$, and $\varepsilon$, a fixed unit vector $e$, and any $\lambda \in\left[1, P^{1 / 2}\right]$, choose $\psi(\lambda)>0$ such that $\psi(\lambda) e \in \Psi_{\varepsilon}\left(S_{1}, \lambda S_{2}\right)$. By the definition of $\Psi_{\varepsilon}\left(S_{1}, \lambda S_{2}\right)$, we have $(6.16 \mathrm{a}, \mathrm{b})$

$$
f_{\varepsilon}\left(\alpha e, d\left(\alpha e, S_{1}\right), d\left(\alpha e, \lambda S_{2}\right)\right)=0=f_{\varepsilon}\left(\beta e, d\left(\beta e, S_{1}\right), d\left(\beta e, \mu S_{2}\right)\right),
$$

for any $1 \leq \lambda \leq \mu \leq P^{1 / 2}$, where $\alpha:=\psi(\lambda) \leq \beta:=\psi(\mu)$ (by Lemma 2.4(c)). Since $\kappa=\kappa(\lambda, \mu):=(\beta / \alpha) \geq 1$, it follows from (6.16a) and Assumption (A2) that

$$
\begin{aligned}
& f_{\varepsilon}\left(\kappa \alpha e, \kappa d\left(\alpha e, S_{1}\right), \kappa d\left(\alpha e, \lambda S_{2}\right)\right) \\
& =f_{\varepsilon}\left(\beta e, d\left(\beta e, \kappa S_{1}\right), d\left(\beta e, \kappa \lambda S_{2}\right)\right) \geq 0 .
\end{aligned}
$$

If we assume that $\kappa \lambda>\mu$, then $d\left(\beta e, \kappa S_{1}\right) \leq d\left(\beta e, S_{1}\right)$ and $d\left(\beta e, \mu S_{2}\right)<$ $d\left(\beta e, \kappa \lambda S_{2}\right)$, from which it follows (by Eq. (6.16b) and Assumption (A1)) that $f_{\varepsilon}\left(\beta e, d\left(\beta e, \kappa S_{1}\right), d\left(\beta e, \kappa \lambda S_{2}\right)\right)<0$. This contradiction (of (6.17)) shows that $\kappa \lambda \leq \mu$ and therefore that $1 \leq \kappa \leq(\mu / \lambda) \leq P^{1 / 2}$ for any $1 \leq \lambda \leq \mu \leq P^{1 / 2}$. By (6.15) and the theorem of the mean, we have that

$$
f_{\varepsilon}\left(x_{0}, p_{1}, q_{1}\right) \leq f_{\varepsilon}\left(x_{0}, p_{0}, q_{0}\right)-C_{0}\left(p_{0}-p_{1}\right)+C_{1}\left(q_{0}-q_{1}\right),
$$

where $x_{0}:=\beta e$, and

$$
\begin{gathered}
0<p_{1}:=d\left(\beta e, \kappa S_{1}\right) \leq p_{0}:=d\left(\beta e, S_{1}\right) \\
0<q_{1}:=d\left(\beta e, \kappa \lambda S_{2}\right) \leq q_{0}:=d\left(\beta e, \mu S_{2}\right), \\
C_{0}=\min \left\{\partial f_{\varepsilon}(x, p, q) / \partial p: \Re_{0} \leq|x| \leq P^{1 / 2} \Re_{1}, a_{0} \leq p, q \leq a_{1}\right\}>0, \\
C_{1}=\max \left\{\left|\partial f_{\varepsilon}(x, p, q) / \partial q\right|: \Re_{0} \leq|x| \leq P^{1 / 2} \Re_{1}, a_{0} \leq p, q \leq a_{1}\right\}>0 .
\end{gathered}
$$

Since $f_{\varepsilon}\left(x_{0}, p_{0}, q_{0}\right)=0$ (by Eq. (6.16b)) and $f_{\varepsilon}\left(x_{0}, p_{1}, q_{1}\right) \geq 0$ (by Eq. $(6.17))$, we have

$$
\left(p_{0}-p_{1}\right) \leq\left(C_{1} / C_{0}\right)\left(q_{0}-q_{1}\right)
$$


On the other hand, it follows from (6.12) that

$$
\begin{aligned}
p_{0}-p_{1} & :=d\left(\beta e, S_{1}\right)-d\left(\beta e, \kappa S_{1}\right) \geq\left(\Re_{0} / P^{1 / 2}\right)(\kappa-1), \\
q_{0}-q_{1}:=d\left(\beta e, \mu S_{2}\right)-d\left(\beta e, \kappa \lambda S_{2}\right) & \leq \Re_{1} P^{1 / 2}(\mu-\kappa \lambda)
\end{aligned}
$$

for $1 \leq \kappa \lambda \leq \mu \leq P^{1 / 2}$ and $\beta e \in E\left(\kappa S_{1}\right) \cap D\left(\kappa \lambda S_{2}\right)$. By substituting (6.20) and (6.21) into (6.19), we obtain $(\kappa-1) \leq\left(\Re_{1} C_{1} P / \Re_{0} C_{0}\right)(\mu-\kappa \lambda)$. Therefore

$$
\kappa:=(\beta / \alpha) \leq\left(\mu+C_{2}\right) /\left(\lambda+C_{2}\right),
$$

where $C_{2}:=\left(\Re_{0} C_{0} / \Re_{1} C_{1} P\right)$. It easily follows from (6.22) (with $\alpha=\psi(\lambda)$, $\beta=\psi(\mu))$ in the limit as $\mu \downarrow \lambda$ that

$$
\psi^{\prime}(\lambda) / \psi(\lambda) \leq 1 /\left(\lambda+C_{2}\right) \leq b / \lambda
$$

for each $\lambda \in\left[1, P^{1 / 2}\right]$, where we define $b=\left(P^{1 / 2} /\left(P^{1 / 2}+C_{2}\right)\right) \in(0,1)$. By integrating (6.23), we conclude that $\psi(\lambda) \leq \psi(1) \lambda^{b}$ for $\lambda \in\left[1, P^{1 / 2}\right]$. The assertion now follows from the observation that the constant " $b$ " in this estimate does not depend on the particular choice of the surfaces $S_{1}, S_{2} \in$ $\mathbb{X}:=\mathbb{X}\left(\Re_{0}, \Re_{1}\right)$ (such that $P S_{1}<S_{2}$ ) or the unit vector $e$.

Lemma 6.7. In the context of $\S 2.3$, for given values $0<\Re_{0} \leq \Re_{1}, P>1$, and $\varepsilon \in(0,1)$, there exists a constant $b \in[1 / 2,1)$ such that

$$
\Psi_{\varepsilon}\left(\widehat{S}_{1}, \widehat{S}_{2}\right) \leq \lambda^{1-B(\mu / \lambda)} \mu^{B(\mu / \lambda)} \Psi_{\varepsilon}\left(S_{1}, S_{2}\right),
$$

for any $\lambda, \mu \in[1, \infty)$ and for any surfaces $S_{1}, S_{2}, \widehat{S}_{1}, \widehat{S}_{2} \in \mathbb{X}:=\mathbb{X}\left(\Re_{0}, \Re_{1}\right)$ such that $P S_{1} \leq S_{2}, P \widehat{S}_{1} \leq \widehat{S}_{2}, \widehat{S}_{1} \leq \lambda S_{1}$, and $\widehat{S}_{2} \leq \mu S_{2}$, where $B(t)=$ $B_{0}:=(1-b) \in(1,1 / 2)$ for $0<t<1$ and $B(t)=B_{1}:=b \in(1 / 2,1)$ for $t \geq 1$.

Proof. We will prove (6.24) only in the case where $1 \leq \mu<\lambda$, since a similar proof applies to the case where $1 \leq \lambda \leq \mu$. Set $\widehat{S}_{2, i}=\min \left(\widehat{S}_{2}, \mu^{i / n} S_{2}\right) \in \mathbb{X}$ and $\widehat{S}_{1, i}=\min \left(\widehat{S}_{1}, \lambda^{i / n} S_{1},(1 / P) \widehat{S}_{2, i}\right) \in \mathbb{X}$, both for $i=0, \ldots, n$, where $n$ denotes a fixed positive integer such that $(\lambda / \mu)^{1 / n} \leq P^{1 / 2}$. It is easily verified that $\widehat{S}_{1, i} \leq(1 / P) \widehat{S}_{2, i}$ for $i=0, \ldots, n$, and that $\widehat{S}_{2, i+1} \leq \mu^{1 / n} \widehat{S}_{2, i}$ and $\widehat{S}_{1, i+1} \leq \lambda^{1 / n} \widehat{S}_{1, i}$, both for all $i=0, \ldots, n-1$. In view of these facts, it follows from Lemma 2.4(c), (e) and Lemma 6.6 that

$$
\begin{aligned}
\Psi_{\varepsilon}\left(\widehat{S}_{1, i+1}, \widehat{S}_{2, i+1}\right) & \leq \Psi_{\varepsilon}\left(\lambda^{1 / n} \widehat{S}_{1, i}, \mu^{1 / n} \widehat{S}_{2, i}\right) \\
& \leq \mu^{1 / n} \Psi_{\varepsilon}\left((\lambda / \mu)^{1 / n} \widehat{S}_{1, i}, \widehat{S}_{2, i}\right) \\
& \leq \mu^{1 / n}(\lambda / \mu)^{b / n} \Psi_{\varepsilon}\left(\widehat{S}_{1, i}, \widehat{S}_{2, i}\right) \\
& =\lambda^{b / n} \mu^{(1-b) / n} \Psi_{\varepsilon}\left(\widehat{S}_{1, i}, \widehat{S}_{2, i}\right)
\end{aligned}
$$


for $i=0, \ldots, n-1$. We conclude from (6.25) by induction that $\Psi_{\varepsilon}\left(\widehat{S}_{1, n}, \widehat{S}_{2, n}\right)$ $\leq \lambda^{b} \mu^{1-b} \Psi_{\varepsilon}\left(\widehat{S}_{1,0}, \widehat{S}_{2,0}\right)$. The assertion follows from this, in view of the facts that $\widehat{S}_{2, n}=\widehat{S}_{2}$ (due to $\widehat{S}_{2} \leq \mu S_{2}$ ), $\widehat{S}_{1, n}=\widehat{S}_{1}$ (due to $\widehat{S}_{1} \leq \lambda S_{1}$ and $\widehat{S}_{1} \leq$ $\left.(1 / P) \widehat{S}_{2}\right), \widehat{S}_{2,0}:=\min \left(\widehat{S}_{2}, S_{2}\right) \leq S_{2}$, and $\widehat{S}_{1,0}:=\min \left(\widehat{S}_{1}, S_{1},(1 / P) S_{2}\right) \leq$ $S_{1}$.

6.8. Proof of Theorem 6.1. For any constants $\lambda>1, \lambda_{ \pm} \geq 1$, and $\kappa_{0} \in(0,1)$ satisfying (6.2) and (6.3), and for any $r \geq 0$ and any triples $\left(S^{-}, S, S^{+}\right),\left(\widehat{S}^{-}, \widehat{S}, \widehat{S}^{+}\right) \in \mathbf{X}_{3}$ satisfying the assumptions (including (6.4)), it follows from Lemma 6.5 that

$$
\Phi_{\varepsilon}^{ \pm}\left(\widehat{S}, \widehat{S}^{ \pm}\right) \leq \lambda_{ \pm}^{r A\left(\lambda_{ \pm} / \lambda\right)} \lambda^{r\left(1-A\left(\lambda_{ \pm} / \lambda\right)\right)} \Phi_{\varepsilon}^{ \pm}\left(S, S^{ \pm}\right) .
$$

Moreover, there is a constant $P_{1}>1$ such that $P_{1} \Phi_{\varepsilon}^{-}\left(S^{-}, S\right) \leq \Phi_{\varepsilon}^{+}\left(S, S^{+}\right)$ and $P_{1} \Phi_{\varepsilon}^{-}\left(\widehat{S}^{-}, \widehat{S}\right) \leq \Phi_{\varepsilon}^{+}\left(\widehat{S}, \widehat{S}^{+}\right)$uniformly for all admissible configurations. Therefore, it follows from Lemma 6.7 (with $P$ replaced by $P_{1}$ ) that

$$
\begin{aligned}
& T_{\varepsilon}\left(\widehat{S}^{-}, \widehat{S}, \widehat{S}^{+}\right) \\
& =\Psi_{\varepsilon}\left(\Phi_{\varepsilon}^{-}\left(\widehat{S}^{-}, \widehat{S}\right), \Phi_{\varepsilon}^{+}\left(\widehat{S}, \widehat{S}^{+}\right)\right) \\
& \leq\left(\lambda^{r}\left(\lambda_{-} / \lambda\right)^{r A\left(\lambda_{-} / \lambda\right)}\right)^{(1-B)}\left(\lambda^{r}\left(\lambda_{+} / \lambda\right)^{r A\left(\lambda_{+} / \lambda\right)}\right)^{B} \\
& \quad \cdot \Psi_{\varepsilon}\left(\Phi_{\varepsilon}^{-}\left(S^{-}, S\right), \Phi_{\varepsilon}^{+}\left(S, S^{+}\right)\right) \\
& \leq\left(\lambda^{\kappa_{0}}\left(\lambda_{-} / \lambda\right)^{A\left(\lambda_{-} / \lambda\right)(1-B)}\left(\lambda_{+} / \lambda\right)^{A\left(\lambda_{+} / \lambda\right) B}\right)^{r} \\
& \quad \cdot \lambda^{\left(1-\kappa_{0}\right) r} T_{\varepsilon}\left(S^{-}, S, S^{+}\right),
\end{aligned}
$$

where $B$ is defined by (6.3). The assertion (6.1) follows directly from (6.2) and (6.27).

\section{Multi-surface problem: Monotonicity and fixed-point properties of the operators $T_{\varepsilon}$.}

7.1. Assumptions. In Problem 1.1, we assume, for given constants $0<\rho_{0} \leq \Re_{0} \leq \Re_{1}$ that $S_{*}^{ \pm} \in \mathbb{X}^{ \pm}\left(\Re_{0}, \Re_{1} ; \rho_{0}\right)$, and that the $C^{1}$-functions $F_{i}(x, p, q): \mathbb{R}^{N} \times \mathbb{R}_{+} \times \mathbb{R}_{+} \rightarrow \mathbb{R}, i=1, \ldots, k$, all satisfy Assumptions (A1)-(A3).

7.2. Inner and outer solutions. We call $\boldsymbol{S} \in \mathbb{Y}$ an inner (resp. outer) solution of Problem 1.3 at $\varepsilon \in(0,1)$ if $\boldsymbol{T}_{\varepsilon}(\boldsymbol{S}) \geq \boldsymbol{S}\left(\operatorname{resp} . \boldsymbol{T}_{\varepsilon}(\boldsymbol{S}) \leq \boldsymbol{S}\right)$. We use $\mathfrak{S}_{\varepsilon}^{-}$(resp. $\mathfrak{S}_{\varepsilon}^{+}$) to denote the family of all inner (outer) solutions at $\varepsilon \in(0,1)$.

\section{Lemma 7.3.}

(a) For any $\varepsilon \in(0,1), \boldsymbol{T}_{\varepsilon}(\boldsymbol{S})$ is a weakly increasing (decreasing) mapping of the family $\mathfrak{S}_{\varepsilon}^{-}$(resp. $\mathfrak{S}_{\varepsilon}^{+}$) into itself. 
(b) For any $\varepsilon \in(0,1)$ and multisurfaces $\boldsymbol{S}_{\varepsilon}^{ \pm} \in \boldsymbol{S}_{\varepsilon}^{ \pm}$, we have that $\boldsymbol{S}_{\varepsilon}^{-} \leq \boldsymbol{S}_{\varepsilon}^{+}$. Therefore, Problem 1.3 has at most one solution at each $\varepsilon \in(0,1)$. If $\widetilde{\boldsymbol{S}}_{\varepsilon}$ solves Problem 1.3 at $\varepsilon \in(0,1)$, then $\boldsymbol{S}_{\varepsilon}^{-} \leq \widetilde{\boldsymbol{S}}_{\varepsilon} \leq \boldsymbol{S}_{\varepsilon}^{+}$for any multisurfaces $\boldsymbol{S}_{\varepsilon}^{ \pm} \in \mathfrak{S}_{\varepsilon}^{ \pm}$.

Proof. Part (a) follows easily from the monotonicity of $\boldsymbol{T}_{\varepsilon}$ (Remark 2.6). For the proof of part (b), let $\boldsymbol{S}_{\varepsilon}^{ \pm}=\left(S_{\varepsilon, 1}^{ \pm}, S_{\varepsilon, 2}^{ \pm}, \ldots, S_{\varepsilon, k}^{ \pm}\right)$. To prove $\boldsymbol{S}_{\varepsilon}^{-} \leq \boldsymbol{S}_{\varepsilon}^{+}$, it suffices to show $\lambda_{i} \leq 1$ for $i=1, \ldots, k$, where we define $\lambda_{i}=\min \{t>$ $\left.0: t S_{\varepsilon, i}^{+} \geq S_{\varepsilon, i}^{-}\right\}$. (We also define $\lambda_{0}=\lambda_{k+1}=1, S_{\varepsilon, 0}^{ \pm}=S_{*}^{-}$, and $S_{\varepsilon, k+1}^{ \pm}=$ $S_{*}^{+}$.) If the assertion is false, then one can choose $j \in\{1, \ldots, k\}$ such that $\lambda_{j}=\max \left\{\lambda_{1}, \ldots, \lambda_{k}\right\}>1$ and either $\lambda_{j-1}<\lambda_{j}$ or $\lambda_{j+1}<\lambda_{j}$. Then we have

$$
\begin{aligned}
S_{\varepsilon, j}^{-} & \leq T_{\varepsilon, j}\left(S_{\varepsilon, j-1}^{-}, S_{\varepsilon, j}^{-}, S_{\varepsilon, j+1}^{-}\right) \\
& <T_{\varepsilon, j}\left(\lambda_{j} S_{\varepsilon, j-1}^{+}, \lambda_{j} S_{\varepsilon, j}^{+}, \lambda_{j} S_{\varepsilon, j+1}^{+}\right) \\
& \leq \lambda_{j} T_{\varepsilon, j}\left(S_{\varepsilon, j-1}^{+}, S_{\varepsilon, j}^{+}, S_{\varepsilon, j+1}^{+}\right) \leq \lambda_{j} S_{\varepsilon, j}^{+},
\end{aligned}
$$

by Lemma 2.5(a), (c) and the definitions of $\mathfrak{S}_{\varepsilon}^{ \pm}$. However, (7.1) shows that $\lambda_{j}$ could have been chosen slightly smaller without violating the requirement that $\lambda_{j} S_{\varepsilon, j}^{+} \geq S_{\varepsilon, j}^{-}$, a contradiction which proves the assertion.

7.4. Additional definitions. In the context of $\S 1.2$, for any given $P>1$, let $\mathbb{Y}(P)$ denote the family of all multisurfaces $\boldsymbol{S}=\left(S_{1}, \ldots, S_{k}\right) \in \mathbb{Y}$ such that $P S_{i} \leq S_{i+1}$ for all $i=0,1, \ldots, k-1$, where $S_{0}:=S_{*}^{-}$and $S_{k+1}:=S_{*}^{+}$. Obviously, $\mathbb{Y}\left(P_{2}\right) \subset \mathbb{Y}\left(P_{1}\right)$ for $1<P_{1} \leq P_{2}$. For given values $\delta_{1}, \ldots, \delta_{k-1}>$ 0 and given multisurfaces $\boldsymbol{S}_{0}^{ \pm} \in \mathbb{Y}$, we use $\mathbb{Y}\left(\boldsymbol{S}_{0}^{-}, \boldsymbol{S}_{0}^{+} ; \delta_{1}, \ldots, \delta_{k-1}\right)$ to denote the family of all multisurfaces $\boldsymbol{S}=\left(S_{1}, \ldots, S_{k}\right) \in \mathbb{Y}$ such that $\boldsymbol{S}_{0}^{-} \leq \boldsymbol{S} \leq \boldsymbol{S}_{0}^{+}$, and such that $d\left(S_{i}, S_{i+1}\right) \geq \delta_{i}$ for all $i=1, \ldots, k-1$.

Lemma 7.5. For any $P>1$ and any $k$-tuple $\boldsymbol{\lambda}=\left(\lambda_{1}, \ldots, \lambda_{k}\right) \in(1, \infty)^{k}$, the multi-surface family $\mathbb{Y}(P)$ is complete in the metric $\boldsymbol{M}_{\boldsymbol{\lambda}}$ (of Eq. (2.1)).

Proof. Let $\left(\boldsymbol{S}_{n}\right)_{n=1}^{\infty}$ denote a Cauchy sequence in $\mathbf{Y}(P)$ relative to the metric $\boldsymbol{M}_{\boldsymbol{\lambda}}$, where $\boldsymbol{S}_{n}=\left(S_{n, 1}, \ldots, S_{n, k}\right)$ for each $n \in \mathbb{N}$. Then for each $i=1, \ldots, k,\left(S_{n, i}\right)_{n=1}^{\infty}$ is a Cauchy sequence in the metric $M$ of Eq. (2.2), which therefore converges in the same metric to a surface $S_{i} \in \mathbb{X}\left(\Re_{0}, \Re_{1}\right)$. We have $\boldsymbol{S}:=\left(S_{1}, \ldots, S_{k}\right) \in \mathbb{Y}(P)$, as follows directly from the fact that $\boldsymbol{S}_{n} \in \mathbb{Y}(P)$ for all $n \in \mathbb{N}$. Obviously $\boldsymbol{M}_{\boldsymbol{\lambda}}\left(\boldsymbol{S}_{n}, \boldsymbol{S}\right) \rightarrow 0$ as $n \rightarrow \infty$.

Lemma 7.6. Given the value $P>1$, there exist two fixed multisurfaces $\boldsymbol{S}_{0}^{ \pm}:=\left(S_{0,1}^{ \pm}, \ldots, S_{0, k}^{ \pm}\right) \in \mathbb{Y}$ such that $P S_{0,1}^{+} \geq S_{*}^{+}$and $S_{0, k}^{-} \leq P S_{*}^{-}$, and such that $\boldsymbol{S}_{0}^{ \pm} \in \boldsymbol{S}_{\varepsilon}^{ \pm}$for all $\varepsilon \in(0,1)$. 
Proof. We will construct only the outer solution $\boldsymbol{S}_{0}^{+}$, since the construction of $\boldsymbol{S}_{0}^{-}$is analogous. Choose $\eta>0$ and the function $g(t):(0, \eta] \rightarrow \mathbb{R}_{+}$so that the second assertion of Theorem 3.1 applies to all $S \in \mathbb{X}^{+}\left(\left(\Re_{0} / P\right), \Re_{1}\right.$; $\left.\left(\rho_{0} / P\right)\right)$. Choose $\eta_{0} \in(0, \eta]$ such that $\left(1+\eta_{0}\right)^{k+1} \leq P$. Choose the values $\lambda_{1}, \ldots, \lambda_{k+1}$ inductively such that $1<\lambda_{i}<1+\eta_{0}$ for $i=1, \ldots, k+1$, and $\lambda_{i}<1+g\left(1-\left(1 / \lambda_{i-1}\right)\right)$ for $i=2, \ldots, k+1$. Define the values $P_{0}, \ldots, P_{k+1}$ by backwards induction such that $P_{k+1}=1$ and $P_{i-1}=\left(P_{i} / \lambda_{i}\right)$ for $i=$ $1, \ldots, k+1$. By assumption, we have $(1 / P) \leq P_{0}<P_{1}<\cdots<P_{k}<$ $P_{k+1}=1$, so that $P_{i} S \in \mathbb{X}^{+}\left(\left(\Re_{0} / P\right), \Re_{1} ;\left(\rho_{0} / P\right)\right)$ for $i=1, \ldots, k$. Define $S_{0,0}^{+}=S_{*}^{-} \leq P_{0} S_{*}^{+}$and $S_{0, i}^{+}=P_{i} S_{*}^{+}$for $i=1, \ldots, k+1$. It follows from Theorem 3.1 (Eq. (3.2)) with $S=S_{0, i}^{+}$that

$$
T_{\varepsilon}\left(S_{0, i-1}^{+}, S_{0, i}^{+}, S_{0, i+1}^{+}\right) \leq T_{\varepsilon}\left(\left(1 / \lambda_{i}\right) S_{0, i}^{+}, S_{0, i}^{+}, \lambda_{i+1} S_{0, i}^{+}\right) \leq S_{0, i}^{+}
$$

for $i=1, \ldots, k$, and for all $\varepsilon \in(0,1)$. The assertion follows.

Lemma 7.7. There exists a constant $P>1$ such that $P S_{*}^{-} \leq \widetilde{S}_{\varepsilon, 1}$ and $P \widetilde{S}_{\varepsilon, k} \leq S_{*}^{+}$for any $\varepsilon \in(0,1)$ and any solution $\widetilde{\boldsymbol{S}}_{\varepsilon}=\left(\widetilde{S}_{\varepsilon, 1}, \ldots, \widetilde{S}_{\varepsilon, k}\right) \in \mathbf{Y}$ of Problem 1.3 at $\varepsilon \in(0,1)$.

Proof. This follows directly from Lemmas 7.3(b) and 7.6.

Lemma 7.8. Let the values $P>1$ and $0<\varepsilon_{0}<\varepsilon_{1}<1$, be given. Then, in the context of Definitions 7.4, the multisurfaces $\boldsymbol{S}_{0}^{ \pm}$and the values $\delta_{1}, \ldots, \delta_{k-1}>0$ can be chosen such that $\mathbb{Y}(P) \subset \mathbb{Y}\left(\boldsymbol{S}_{0}^{-}, \boldsymbol{S}_{0}^{+} ; \delta_{1}, \ldots, \delta_{k-1}\right)$, and such that the transformation $\boldsymbol{T}_{\varepsilon}$ maps the family $\mathbb{Y}\left(\boldsymbol{S}_{0}^{-}, \boldsymbol{S}_{0}^{+} ; \delta_{1}, \ldots, \delta_{k-1}\right)$ into itself for all $\varepsilon \in\left[\varepsilon_{0}, \varepsilon_{1}\right]$.

Proof. Choose $\boldsymbol{S}_{0}^{ \pm}:=\left(S_{0,1}^{ \pm}, \ldots, S_{0, k}^{ \pm}\right) \in \mathbb{Y}$ such that $P S_{0,1}^{+} \geq S_{*}^{+}, S_{0, k}^{-} \leq$ $P S_{*}^{-}$, and $\boldsymbol{S}_{0}^{ \pm} \in \mathfrak{S}_{\varepsilon}^{ \pm}$for all $\varepsilon \in(0,1)$ (as in Lemma 7.6). Let $\kappa>0$ and the function $h_{0}\left(t, \varepsilon_{0}, \varepsilon_{1}\right):(0, \kappa] \rightarrow \mathbb{R}_{+}$be as introduced in Theorem 5.1. Set $\widehat{\kappa}=\min \left\{\kappa, \Re_{0}(P-1)\right\}$. Choose the values $\delta_{1}, \ldots, \delta_{k-1} \in(0, \widehat{\kappa}]$ inductively such that $\delta_{i} \in\left(0, h_{0}\left(\delta_{i-1}, \varepsilon_{0}, \varepsilon_{1}\right)\right]$ for $i=1, \ldots, k-1$, where $\delta_{0}:=d\left(S_{*}^{-}, S_{0,1}^{-}\right)$, or such that $\delta_{i} \in\left(0, h_{0}\left(\delta_{i+1}, \varepsilon_{0}, \varepsilon_{1}\right)\right]$ for $i=2, \ldots, k-1$, where $\delta_{k}:=d\left(S_{0, k}^{+}, S_{*}^{+}\right)$. We have $\mathbb{Y}(P) \subset \widehat{\mathbb{Y}}:=\left\{\boldsymbol{S} \in \mathbb{Y}: \boldsymbol{S}_{0}^{-} \leq \boldsymbol{S} \leq \boldsymbol{S}_{0}^{+}\right\}$, since $S_{0, i}^{-} \leq S_{0, k}^{-} \leq P S_{*}^{-} \leq S_{i}$ and $P S_{i} \leq S_{*}^{+} \leq P S_{0,1}^{+} \leq P S_{0, i}^{+}$for any $\boldsymbol{S}=$ $\left(S_{1}, \ldots, S_{k}\right) \in \mathbb{Y}(P)$. Therefore $\mathbb{Y}(P) \subset \widetilde{\mathbb{Y}}:=\mathbb{Y}\left(\boldsymbol{S}_{0}^{-}, \boldsymbol{S}_{0}^{+} ; \delta_{1}, \ldots, \delta_{k-1}\right)$, since Lemma 3.6 implies that $d\left(S_{i}, S_{i+1}\right) \geq \Re_{0}(P-1) \geq \delta_{i}$ for $\boldsymbol{S} \in \mathbb{Y}(P)$ and $i=1, \ldots, k-1$. Also, $\boldsymbol{T}_{\varepsilon}(\boldsymbol{S}): \widehat{\mathbb{Y}} \rightarrow \widehat{\mathbb{Y}}$ for $\varepsilon \in(0,1)$, due to Remark 2.6 and the fact that $\boldsymbol{S}_{0}^{ \pm} \in \boldsymbol{S}_{\varepsilon}^{ \pm}$, and we have $d\left(T_{\varepsilon, i}(\boldsymbol{S}), T_{\varepsilon, i+1}(\boldsymbol{S})\right) \geq \delta_{i}$ for $\boldsymbol{S} \in \widetilde{\mathbb{Y}}$ and $i=1, \ldots, k-1$, by Theorem 5.1.

Lemma 7.9. Let the values $0<\varepsilon_{0}<\varepsilon_{1}<1$ and $P>1$ be given. Then there exists a value $P_{1}>1$ such that $\boldsymbol{T}_{\varepsilon}^{n}(\boldsymbol{S}) \in \mathbb{Y}\left(P_{1}\right)$ for all $\boldsymbol{S} \in \mathbb{Y}(P)$, all 
$\varepsilon \in\left[\varepsilon_{0}, \varepsilon_{1}\right]$, and all $n \in \mathbb{N}$, where $\boldsymbol{T}_{\varepsilon}^{n}$ refers to the $n$-fold application of the operator $\boldsymbol{T}_{\varepsilon}$.

Proof. In the context of $\S \S 7.4,7.8$, we have $\mathbb{Y}(P) \subset \mathbb{Y}\left(\boldsymbol{S}_{0}^{-}, \boldsymbol{S}_{0}^{+} ; \delta_{1}, \ldots\right.$, $\left.\delta_{k-1}\right)$, for suitable multisurfaces $\boldsymbol{S}_{0}^{ \pm} \in \mathbb{Y}$ and values $\delta_{1}, \ldots, \delta_{k-1}$. On the other hand, we have $\mathbb{Y}\left(\boldsymbol{S}_{0}^{-}, \boldsymbol{S}_{0}^{+} ; \delta_{1}, \ldots, \delta_{k-1}\right) \subset \mathbb{Y}\left(P_{1}\right)$ provided that $P_{1}>$ 1 is chosen sufficiently close to 1 .

Theorem 7.10. For given $0<\Re_{0}<\Re_{1}, P>1$, and $\varepsilon \in(0,1)$, one can choose a value $\kappa_{0} \in(0,1)$ and a $k$-tuple $\boldsymbol{\lambda}=\left(\lambda_{1}, \ldots, \lambda_{k}\right) \in(1, \infty)^{k}$ such that

$$
\boldsymbol{M}_{\boldsymbol{\lambda}}\left(\boldsymbol{T}_{\varepsilon}(\boldsymbol{S}), \boldsymbol{T}_{\varepsilon}(\widehat{\boldsymbol{S}})\right) \leq\left(1-\kappa_{0}\right) \boldsymbol{M}_{\boldsymbol{\lambda}}(\boldsymbol{S}, \widehat{\boldsymbol{S}})
$$

uniformly for all multisurfaces $\boldsymbol{S}, \widehat{\boldsymbol{S}} \in \mathbb{Y}(P)$.

Theorem 7.11. For any given $\varepsilon \in(0,1)$ and any sufficiently small $P>1$ (small enough so that $\mathbb{Y}(P) \neq \emptyset$ ), there exists a (unique) solution $\widetilde{\boldsymbol{S}}_{\varepsilon} \in \mathbb{Y}$ of Problem 1.3 at $\varepsilon$, and there exist a constant $\kappa_{0} \in(0,1)$ and a $k$-tuple $\boldsymbol{\lambda}=\left(\lambda_{1}, \ldots, \lambda_{k}\right) \in(1, \infty)^{k}$ such that

$$
\boldsymbol{M}_{\boldsymbol{\lambda}}\left(\boldsymbol{T}_{\varepsilon}^{n}(\boldsymbol{S}), \widetilde{\boldsymbol{S}}_{\varepsilon}\right) \leq\left(\left(1-\kappa_{0}\right)^{n} / \kappa_{0}\right) \boldsymbol{M}_{\boldsymbol{\lambda}}\left(\boldsymbol{T}_{\varepsilon}(\boldsymbol{S}), \boldsymbol{S}\right)
$$

uniformly for all $\boldsymbol{S} \in \mathbb{Y}(P)$ and all $n \in \mathbb{N}$. The estimate (1.9) follows by the equivalence of the metrics $\boldsymbol{M}$ and $\boldsymbol{M}_{\boldsymbol{\lambda}}$. Thus, the iterates $\boldsymbol{T}_{\varepsilon}^{n}(\boldsymbol{S}), n \in \mathbb{N}$, converge geometrically to the fixed point $\widetilde{\boldsymbol{S}}_{\varepsilon}$ of $\boldsymbol{T}_{\varepsilon}$ as $n \rightarrow \infty$. Moreover, the iterates converge monotonically to $\widetilde{\boldsymbol{S}}_{\varepsilon}$ as $n \rightarrow \infty$ provided that $\boldsymbol{S} \in$ $\mathfrak{S}_{\varepsilon}^{-} \cup \mathfrak{S}_{\varepsilon}^{+}$.

Proof. For given, small $P>1$, there exists a value $P_{1}>1$ such that $\mathbb{Y}\left(P_{1}\right)$ contains the image of $\mathbb{Y}(P)$ under all multiple applications of the transformation $\boldsymbol{T}_{\varepsilon}$. Then (7.2) holds for all $\boldsymbol{S} \in \mathbb{Y}\left(P_{1}\right)$, where $\kappa_{0} \in(0,1)$ and the $k$-tuple $\boldsymbol{\lambda}=\left(\lambda_{1}, \ldots, \lambda_{k}\right)$ are chosen as in Theorem 7.10 , but relative to the family $\mathbb{Y}\left(P_{1}\right)$. At this point, the assertion follows by the classical proof of the contraction-mapping theorem (see $[\mathbf{1 4}, \S 5.1]$ ). In particular, it follows from (7.2) that

$$
\boldsymbol{M}_{\boldsymbol{\lambda}}\left(\boldsymbol{T}_{\varepsilon}^{n}(\boldsymbol{S}), \boldsymbol{T}_{\varepsilon}^{m}(\boldsymbol{S})\right) \leq\left(\left(1-\kappa_{0}\right)^{n} / \kappa_{0}\right) \boldsymbol{M}_{\boldsymbol{\lambda}}\left(\boldsymbol{T}_{\varepsilon}(\boldsymbol{S}), \boldsymbol{S}\right)
$$

uniformly for all $\boldsymbol{S} \in \mathbb{Y}(P)$ and $m, n \in \mathbb{N}$ such that $n<m$. For any $\boldsymbol{S} \in \mathbb{Y}(P)$, Eq. (7.4) implies that $\left(\boldsymbol{T}_{\varepsilon}^{n}(\boldsymbol{S})\right)_{n=1}^{\infty}$ is a Cauchy sequence, which therefore must converge to the solution $\widetilde{\boldsymbol{S}}_{\varepsilon} \in \mathbb{Y}\left(P_{1}\right)$ of Problem 1.3 (which is unique by Lemma 7.3(b), or by Eq. (7.2)). The assertion (7.3) follows directly from (7.4) in the limit as $m \rightarrow \infty$. For the case where $\boldsymbol{S} \in \mathfrak{S}_{\varepsilon}^{-} \cup \mathfrak{S}_{\varepsilon}^{+}$, the monotonicity of the convergence follows from the monotonicity of the mapping $\boldsymbol{T}_{\varepsilon}: \mathbb{Y} \rightarrow \mathbb{Y}$ (see Lemma 7.3(a)). 
7.12. Proof of Theorem 7.10. We will choose a $k$-tuple $\boldsymbol{\lambda}=\left(\lambda_{1}, \ldots, \lambda_{k}\right)$ and a value $\kappa_{0}>0$ such that

$$
1=: \lambda_{0}<\lambda_{1}<\cdots<\lambda_{m-1}<\lambda_{m}>\lambda_{m+1}>\cdots>\lambda_{k}>\lambda_{k+1}:=1,
$$

where either $k=2 m$ or $k=2 m-1$, and such that

$$
T_{\varepsilon, i}\left(\widehat{S}_{i-1}, \widehat{S}_{i}, \widehat{S}_{i+1}\right) \leq \lambda_{i}^{\left(1-\kappa_{0}\right) r} T_{\varepsilon, i}\left(S_{i-1}, S_{i}, S_{i+1}\right), \quad i=1, \ldots, k,
$$

for any $r \geq 0$, and for any ordered $k$-tuples $\boldsymbol{S}=\left(S_{1}, \ldots, S_{k}\right), \widehat{\boldsymbol{S}}=\left(\widehat{S}_{1}, \ldots, \widehat{S}_{k}\right)$ $\in \mathbb{Y}(P)$ such that $\widehat{S}_{i} \leq \lambda_{i}^{r} S_{i}$ for $i=1, \ldots, k$ (where $S_{0}=\widehat{S}_{0}=S_{*}^{-}$and $S_{k+1}=\widehat{S}_{k+1}=S_{*}^{+}$). The assertion follows directly from this (see Remark 6.2). For $\boldsymbol{\lambda}=\left(\lambda_{1}, \ldots, \lambda_{k}\right)$ in the form given in (7.5), the condition (7.6) holds for $i \in\{1, \ldots, k\}$ if

$$
\begin{aligned}
\lambda_{i-1}^{\alpha} \lambda_{i+1}^{\beta} & \leq \lambda_{i}^{\left(1-\kappa_{1}\right)} \\
\lambda_{i-1}^{\beta} \lambda_{i+1}^{\alpha} & \leq \lambda_{i}^{\left(1-\kappa_{1}\right)} \\
\lambda_{m-1}^{(1-B)} \lambda_{m+1}^{B} & \leq \lambda_{m}^{\left(1-\kappa_{2}\right)},
\end{aligned}
$$

where $\alpha=A_{0} B_{0} /\left(A_{0} B_{0}+B_{1}\right) \in(0,1 / 2), \beta=(1-\alpha) \in(1 / 2,1), \kappa_{1}=$ $\kappa_{0} /\left(A_{0} B_{0}+B_{1}\right)>0, B \in\left\{B_{0}, B_{1}\right\}$, and $\kappa_{2}=\kappa_{0} / A_{0}>0$. These sufficient conditions for (7.6) follow from Theorem 6.1 and Remark 6.3. It is easily seen (by direct substitution) that if $\kappa_{0}>0$ is sufficiently small (so that $\left.\kappa_{1} \in\left(0,1-2(\alpha \beta)^{1 / 2}\right)\right)$, then the conditions in (7.7) and (7.8) are all satisfied as equalities by setting

$$
\begin{gathered}
\ln \left(\lambda_{i}\right)=\left(\left(R_{2}^{i}-R_{1}^{i}\right) /\left(R_{2}^{m}-R_{1}^{m}\right)\right) \ln \left(\lambda_{m}\right) \text { for } i=0,1, \ldots, m \\
\ln \left(\lambda_{i}\right)=\left(\left(R_{2}^{k+1-i}-R_{1}^{k+1-i}\right) /\left(R_{2}^{k+1-m}-R_{1}^{k+1-m}\right)\right) \ln \left(\lambda_{m}\right) \\
\text { for } i=m, \ldots, k+1
\end{gathered}
$$

where the values $R_{1}=R_{1}\left(\kappa_{0}\right) \in\left(0,(\alpha / \beta)^{1 / 2}\right)$ and $R_{2}=R_{2}\left(\kappa_{0}\right) \in\left((\alpha / \beta)^{1 / 2}, 1\right)$ are the two solutions of the equation

$$
\phi(t):=(\alpha / t)+\beta t=1-\kappa_{1},
$$

and where the constant $\lambda_{m}>1$ is arbitrary. A simple argument based on (7.10) and (7.11) shows that the monotonicity requirements of (7.5) are all satisfied if

$$
\left(R_{2} / R_{1}\right)^{m}<\left(\left(1-R_{1}\right) /\left(1-R_{2}\right)\right) .
$$

Also, it follows from (7.12) that $R_{1}\left(\kappa_{0}\right) \downarrow(\alpha / \beta)$ and $R_{2}\left(\kappa_{0}\right) \uparrow 1$ as $\kappa_{0} \downarrow 0$. Therefore, (7.13) is obviously satisfied if the constant $\kappa_{0}>0$ is sufficiently small. It is easy to see by using (7.10) and (7.11) that the inequality (7.9) is satisfied if

$$
(1-B)\left(\frac{\left(R_{2}^{m-1}-R_{1}^{m-1}\right)}{\left(R_{2}^{m}-R_{1}^{m}\right)}\right)+B\left(\frac{\left(R_{2}^{k-m}-R_{1}^{k-m}\right)}{\left(R_{2}^{k+1-m}-R_{1}^{k+1-m}\right)}\right) \leq\left(1-\kappa_{2}\right)
$$


for $B \in\left\{B_{0}, B_{1}\right\}$. Obviously, (7.14) holds if

$$
\left(\left(R_{2}^{i-1}-R_{1}^{i-1}\right) /\left(R_{2}^{i}-R_{1}^{i}\right)\right) \leq\left(1-\kappa_{2}\right)
$$

for $i=m$ and $i=k+1-m$. A sufficient condition for (7.15) is that

$$
\left(R_{2} / R_{1}\right)^{i-1} \leq\left(\left[1-\left(1-\kappa_{2}\right) R_{1}\right] /\left[1-\left(1-\kappa_{2}\right) R_{2}\right]\right) .
$$

Therefore, (7.14) and (7.9) both hold in each of the cases $k=2 m-1$ and $k=2 m$, provided that

$$
\left[R_{2} / R_{1}\right]^{m} \leq\left(\left[1-\left(1-\kappa_{2}\right) R_{1}\right] /\left[1-\left(1-\kappa_{2}\right) R_{2}\right]\right) .
$$

However, (7.17) holds for sufficiently small $\kappa_{2}>0$, by an argument closely related to the validation of (7.13) for small $\kappa_{0}>0$. Therefore, if $\kappa_{0}>0$ is sufficiently small, than the requirements (7.7), (7.8), and (7.9) are all satisfied by choosing the values $\lambda_{i}, i=1, \ldots, k$, as in (7.10) and (7.11).

\section{A contraction property for 3-surface configurations involving classical solutions.}

Theorem 8.1. In the context of $\S 2.3$, for given values $0<\rho \leq \Re_{0} \leq$ $\Re_{1}$, let $\left(S^{-}, S, S^{+}\right) \in \mathbf{X}_{3}\left(\Re_{0}, \Re_{1}\right)$ denote an ordered triple such that $S \in$ $\mathbb{X}\left(\Re_{0}, \Re_{1} ; \rho\right)$ and such that the joining condition: $F\left(x,\left|\nabla U^{-}(x)\right|,\left|\nabla U^{+}(x)\right|\right)$ $=0$ holds for all $x \in S$, where we define $U^{ \pm}(x):=U^{ \pm}\left(S, S^{ \pm} ; x\right)$. Let the values $0<\mu_{0}<1<\mu_{1}, C_{0}>1$, and $0<P_{-}<1<P_{+}$be chosen such that $S^{-}<P_{-} S<S<P_{+} S<S^{+}$. For any $\mu>0$, let

$$
S^{-}(\mu)=\min \left(P_{-} S, \mu S^{-}\right) ; \quad S^{+}(\mu)=\max \left(P_{+} S, \mu S^{+}\right)
$$

(as in Theorem 4.3). Then there exist values $\kappa_{0}>0,0<A_{0} \leq A_{1}, B_{0} \in$ $(0,1 / 2), B_{1}=\left(1-B_{0}\right) \in(1 / 2,1)$, and a null function $z(\cdot)$ (all dependent only on $F, S, S^{ \pm}$, and the values $\left.\mu_{0}, \mu_{1}, C_{0}, P_{ \pm}\right)$such that, for any given $r_{0}>0$, we have

$$
\begin{aligned}
T_{\varepsilon}\left(S^{-}\left(\mu_{-}^{r}\right), S, S^{+}\left(\mu_{+}^{r}\right)\right) & \leq \exp \left(\varepsilon\left(z(\varepsilon)-\kappa_{0} r\right)\right) S, \\
T_{\varepsilon}\left(S^{-}\left(\mu_{-}^{-r}\right), S, S^{+}\left(\mu_{+}^{-r}\right)\right) & \geq \exp \left(\varepsilon\left(\kappa_{0} r-z(\varepsilon)\right)\right) S,
\end{aligned}
$$

both uniformly for all $\varepsilon \in(0,1)$, all $r \in\left[0, r_{0}\right]$, and all values $\mu_{ \pm} \in$ $\left[\mu_{0}^{q_{0}}, \mu_{1}^{q_{0}}\right] \cap\left[\mu_{1}^{-q_{0}}, \mu_{0}^{-q_{0}}\right]$ (where $\left.q_{0}:=1 / r_{0}\right)$ such that

$$
C_{0}\left(\mu_{-}\right)^{(1-B) A\left(\mu_{-}\right)}\left(\mu_{+}\right)^{B A\left(\mu_{+}\right)} \leq 1 .
$$

Here, we define

$$
B=B\left(\left(\mu_{+}\right)^{A\left(\mu_{+}\right)} /\left(\mu_{-}\right)^{A\left(\mu_{-}\right)}\right),
$$


and we use $A(t), B(t): \mathbb{R}_{+} \rightarrow \mathbb{R}_{+}$to denote functions such that $A(t)=A_{0}$ and $B(t)=B_{0}$ both for $0<t \leq 1$, and $A(t)=A_{1}$ and $B(t)=B_{1}$ both for $t>1$.

Corollary 8.2. In the context of Theorem 8.1, let the values $1<\Lambda_{0}<\Lambda_{1}$ and $\kappa>0$ be given (instead of $\mu_{0}, \mu_{1}$, and $C_{0}$ ). Let the functions $A(t), B(t)$ : $\mathbb{R}_{+} \rightarrow \mathbb{R}_{+}$be as defined previously, where we choose $\mu_{0}=\left(1 / \Lambda_{1}\right)<1<$ $\mu_{1}=\left(\Lambda_{1} / \Lambda_{0}\right)$. Then, given $r_{0}>0$, there exist a value $\kappa_{0}>0$ and a null function $z(\cdot)$ such that

$$
\lambda^{-\left(1-\kappa_{0} \varepsilon\right) r-\varepsilon z(\varepsilon)} S \leq T_{\varepsilon}\left(\widehat{S}^{-}, \widehat{S}, \widehat{S}^{+}\right) \leq \lambda^{\left(1-\kappa_{0} \varepsilon\right) r+\varepsilon z(\varepsilon)} S
$$

uniformly for all $\varepsilon \in(0,1)$, all $r \in\left[0, r_{0}\right]$, all $\lambda \in\left[\Lambda_{0}, \Lambda_{1}\right]$ and $\lambda_{ \pm} \in\left[1, \Lambda_{1}\right]$ such that the corresponding values $\mu_{ \pm}:=\left(\lambda_{ \pm} / \lambda\right) \in\left[\mu_{0}^{q_{0}}, \mu_{1}^{q_{0}}\right] \cap\left[\mu_{1}^{-q_{0}}, \mu_{0}^{-q_{0}}\right]$ satisfy (8.4) and (8.5) with $C_{0}=\lambda^{\kappa}$, and all ordered triples $\left(\widehat{S}^{-}, \widehat{S}, \widehat{S}^{+}\right) \in$ $\mathbf{X}_{3}\left(\Re_{0}, \Re_{1}\right)$ such that

$$
\lambda^{-r} S \leq \widehat{S} \leq \lambda^{r} S ; \lambda^{-r} S^{ \pm}\left(\mu_{ \pm}^{-r}\right) \leq \widehat{S}^{ \pm} \leq \lambda^{r} S^{ \pm}\left(\mu_{ \pm}^{r}\right),
$$

where the surfaces $S^{ \pm}(\mu)$ are defined in (8.1).

8.3. Remarks. (a) For any $\left(S^{-}, S, S^{+}\right) \in \mathbf{X}_{3}\left(\Re_{0}, \Re_{1}\right)$ such that $S \in$ $\mathbb{X}\left(\Re_{0}, \Re_{1} ; \rho\right)$, we have $U^{ \pm}=U^{ \pm}\left(S, S^{ \pm} ; \cdot\right) \in C^{1, \alpha}\left(S \cup \Omega\left(S, S^{ \pm}\right)\right)$for any $\alpha \in(0,1)$, as follows from [14, Thm. 8.34]. We also have $\nabla U^{ \pm}(x) \neq 0$ on $S$ by the Hopf boundary-point lemma [14, Lemma 3.4]. Since we assume $S \in \mathbb{X}\left(\Re_{0}, \Re_{1} ; \rho\right)$ in Theorem 8.1, the functions $\left|\nabla U^{ \pm}(x)\right|: S \rightarrow \mathbb{R}_{+}$ appearing in the joining condition: $F\left(x,\left|\nabla U^{-}(x)\right|,\left|\nabla U^{+}(x)\right|\right)=0$ are automatically Hölder continuous and strictly positive. (b) The main assumption in Corollary 8.2 (that $\mu_{ \pm}:=\left(\lambda_{ \pm} / \lambda\right.$ ) satisfy (8.4) and (8.5) with $C_{0}=\lambda^{\kappa}$ ) reduces to the requirement that $\lambda_{-}^{\alpha} \lambda_{+}^{\beta} \leq \lambda^{1-\kappa_{1}}$ in the case $\lambda_{-}<\lambda<\lambda_{+}$, and to the requirement that $\lambda_{-}^{\beta} \lambda_{+}^{\alpha} \leq \lambda^{1-\kappa_{1}}$ in the case $\lambda_{-}>\lambda>\lambda_{+}$, where we set $\alpha=\left(A_{0} B_{0} /\left(A_{0} B_{0}+A_{1} B_{1}\right)\right) \in(0,1 / 2), \beta=(1-\alpha) \in(1 / 2,1)$, and $\kappa_{1}=\kappa /\left(A_{0} B_{0}+A_{1} B_{1}\right)$. For the case where $\lambda_{-}\langle\lambda\rangle \lambda_{+}$, the same condition reduces to the requirement that $\lambda_{-}^{1-B_{i}} \lambda_{+}^{B_{i}} \leq \lambda^{1-\kappa_{2}}$ for $i=0,1$, where $\kappa_{2}=\left(\kappa / A_{0}\right)$.

Theorem 8.4. In the context of $\S 2.3$, for given values $0<\rho \leq \Re_{0} \leq$ $\Re_{1}$, let $\left(S^{-}, S, S^{+}\right) \in \mathbf{X}_{3}\left(\Re_{0}, \Re_{1}\right)$ denote a particular ordered triple such that $S \in \mathbb{X}\left(\Re_{0}, \Re_{1} ; \rho\right)$, and such that the joining condition: $F\left(x,\left|\nabla U^{-}(x)\right|\right.$, $\left.\left|\nabla U^{+}(x)\right|\right)=0$ holds for all $x \in S$, where $U^{ \pm}(x):=U^{ \pm}\left(S, S^{ \pm} ; x\right)$. Given the values $1<\Lambda_{0}<\Lambda_{1}<\infty$, choose fixed values $0<\delta_{-} \leq \widehat{\delta}_{-}<1 / 2$, $0<\delta_{+} \leq \widehat{\delta}_{+}<1 / 2$, and $C>0$ such that

$$
T_{\varepsilon}\left(\widehat{S}, P_{-} \widehat{S}, P_{-} \widehat{P}_{-} \widehat{S}\right) \geq \lambda^{C \varepsilon} P_{-} \widehat{S}
$$

for all $\widehat{S} \in \mathbb{X}^{-}\left(\Re_{0}, \Re_{1} ; \rho\right), \varepsilon \in(0,1)$, and $\lambda \in\left[\Lambda_{0}, \Lambda_{1}\right]$, and

$$
T_{\varepsilon}\left(\widehat{P}_{+} P_{+} \widehat{S}, P_{+} \widehat{S}, \widehat{S}\right) \leq \lambda^{-C \varepsilon} P_{+} \widehat{S}
$$


for all $\widehat{S} \in \mathbb{X}^{+}\left(\Re_{0}, \Re_{1} ; \rho\right), \varepsilon \in(0,1)$ and $\lambda \in\left[\Lambda_{0}, \Lambda_{1}\right]$, where $P_{ \pm}=1 \mp \delta_{ \pm}$ and $\widehat{P}_{ \pm}=1 \mp \widehat{\delta}_{ \pm}$(see Theorem 3.1 and Remark 3.2$)$. Given the values $\kappa>0$ and $r_{0}>0$, let $\mathfrak{M}$ denote the set of all ordered triples $\left(\lambda_{-}, \lambda, \lambda_{+}\right)$such that $\lambda \in\left[\Lambda_{0}, \Lambda_{1}\right]$ and $\lambda_{ \pm} \in\left[1, \Lambda_{1}\right]$, and such that the corresponding values $\mu_{ \pm}:=\left(\lambda_{ \pm} / \lambda\right) \in\left[\mu_{0}^{q_{0}}, \mu_{1}^{q_{0}}\right] \cap\left[\mu_{1}^{-q_{0}}, \mu_{0}^{-q_{0}}\right]$ (where $\left.q_{0}:=1 / r_{0}\right)$ satisfy (8.4) and (8.5) with $C_{0}=\lambda^{\kappa}$. Then there exist a value $\kappa_{0}>0$ and a null function $z(\cdot)$ such that

$$
\lambda^{\left(\kappa_{0} r-z(\varepsilon)\right) \varepsilon} S_{-}(r) \leq T_{\varepsilon}\left(\widehat{S}^{-}, \widehat{S}, \widehat{S}^{+}\right) \leq \lambda^{\left(z(\varepsilon)-\kappa_{0} r\right) \varepsilon} S_{+}(r),
$$

uniformly for any $\left(\lambda_{-}, \lambda, \lambda_{+}\right) \in \mathfrak{M}$, any $\varepsilon \in(0,1)$, any $r \in\left[0, r_{0}\right]$, and any pair of ordered triples $\left(S_{-}^{-}(r), S_{-}(r), S_{-}^{+}(r)\right) \in \mathbf{X}_{3}^{-}\left(\Re_{0}, \Re_{1} ; \rho\right),\left(S_{+}^{-}(r), S_{+}(r)\right.$, $\left.S_{+}^{+}(r)\right) \in \mathbf{X}_{3}^{+}\left(\Re_{0}, \Re_{1} ; \rho\right)$ such that

$$
\begin{gathered}
\lambda_{-}^{-r} S^{-} \leq S_{-}^{-}(r) \leq S^{-} ; \quad S_{-}(r):=\max \left(\lambda^{-r} S, P_{-} S_{-}^{-}(r)\right) ; \\
S_{-}^{+}(r):=\max \left(\lambda_{+}^{-r} S^{+}, \widehat{P}_{-} S_{-}(r)\right), \\
S^{+} \leq S_{+}^{+}(r) \leq \lambda_{+}^{r} S^{+} ; \quad S_{+}(r):=\min \left(\lambda^{r} S, P_{+} S_{+}^{+}(r)\right) ; \\
S_{+}^{-}(r):=\min \left(\lambda_{-}^{r} S^{-}, \widehat{P}_{+} S_{+}(r)\right),
\end{gathered}
$$

both corresponding to the same triple $\left(\lambda_{-}, \lambda, \lambda_{+}\right) \in \mathfrak{M}$, and all ordered triples $\left(\widehat{S}^{-}, \widehat{S}, \widehat{S}^{+}\right) \in \mathbf{X}_{3}\left(\Re_{0}, \Re_{1}\right)$ such that

$$
S_{-}(r) \leq \widehat{S} \leq S_{+}(r) ; \quad S_{-}^{ \pm}(r) \leq \widehat{S}^{ \pm} \leq S_{+}^{ \pm}(r) .
$$

Lemma 8.5. In the context of Theorem 8.1 , let $Q^{ \pm}\left(\mu_{ \pm} ; x\right):=\left(1 / \mid \nabla U^{ \pm}\left(\mu_{ \pm}\right.\right.$; $x) \mid)$ on $S$ for any $\mu_{ \pm} \in I:=\left[\mu_{0}, \mu_{1}\right]$, where $U^{ \pm}\left(\mu_{ \pm} ; x\right):=U^{ \pm}\left(S, S^{ \pm}\left(\mu_{ \pm}\right) ; x\right)$ in $\mathrm{Cl}\left(\Omega\left(S, S^{ \pm}\left(\mu_{ \pm}\right)\right)\right)$, and the surfaces $S^{ \pm}\left(\mu_{ \pm}\right)$are defined by Eq. (8.1). For any vector $e \in \partial B(0 ; 1)$, any values $\mu_{ \pm} \in I$, and any $\varepsilon \in(0,1)$, let the values $\alpha_{0}=\alpha_{0}(e)>0$ and $\alpha(e, \varepsilon)=\alpha\left(\mu_{-}, \mu_{+}, e ; \varepsilon\right)>0$ be uniquely determined by the requirement that $x_{0}=x_{0}(e):=\alpha_{0} e \in S$ and $x(e, \varepsilon):=\alpha(e, \varepsilon) e \in$ $T_{\varepsilon}\left(S^{-}\left(\mu_{-}\right), S, S^{+}\left(\mu_{+}\right)\right)$. Let the value $h=h\left(\mu_{-}, \mu_{+}, e\right)$ be the unique solution (in the open interval $\left.J\left(\mu_{-}, \mu_{+}, e\right):=\left(-\left[Q^{-}\left(\mu_{-} ; x_{0}\right) / \theta\right],\left[Q^{+}\left(\mu_{+} ; x_{0}\right) / \theta\right]\right)\right)$ of the equation

(8.14)

$$
\phi(h)=\phi\left(\mu_{-}, \mu_{+}, e ; h\right):=f\left(x_{0}, Q^{-}\left(\mu_{-} ; x_{0}\right)+\theta h, Q^{+}\left(\mu_{+} ; x_{0}\right)-\theta h\right)=0,
$$

where $f(x, p, q):=F(x, 1 / p, 1 / q), \nu=\nu(e)$ denotes the exterior unit normal to $S$ at the point $x_{0}=x_{0}(e)=\alpha_{0} e \in S$, and $\theta=\theta(e)=\nu \cdot e$. Then

$$
\left|\left[\left(\alpha\left(\mu_{-}, \mu_{+}, e ; \varepsilon\right)-\alpha_{0}(e)\right) / \varepsilon\right]-h\left(\mu_{-}, \mu_{+}, e\right)\right| \leq z(\varepsilon)
$$

as $\varepsilon \downarrow 0$, where the estimate is uniform over all unit vectors $e$ and all values $\mu_{ \pm} \in I$. 
Proof. For any $x_{0}=\alpha_{0} e \in S$ and $\mu_{ \pm} \in I$, Equation (8.14) has one and only one solution $h\left(\mu_{-}, \mu_{+}, e\right) \in J\left(\mu_{-}, \mu_{+}, e\right)$, by Lemma 3.3(a). By a simple barrier argument, there exist constants $0<C_{0} \leq C_{1}$ such that

$$
C_{0} \leq Q^{ \pm}\left(\mu_{ \pm} ; x_{0}\right) \leq C_{1}
$$

for all $\mu_{ \pm} \in I$ and $x_{0} \in S$. Therefore

$$
\begin{aligned}
2 C_{0} & \leq Q^{-}\left(\mu_{-} ; x_{0}\right)+Q^{+}\left(\mu_{+} ; x_{0}\right) \\
& =\left(Q^{-}\left(\mu_{-} ; x_{0}\right)+\theta t\right)+\left(Q^{+}\left(\mu_{+} ; x_{0}\right)-\theta t\right) \leq 2 C_{1}
\end{aligned}
$$

uniformly for all $\mu_{ \pm} \in I, x_{0} \in S$, and $t \in J\left(\mu_{-}, \mu_{+}, e\right)$. In view of (8.17) and Lemma 3.3(b), there exist constants $0<C_{2} \leq C_{3}$ such that

$$
C_{2} \leq\left(Q^{ \pm}\left(\mu_{ \pm} ; x_{0}\right) \mp \theta h\right) \leq C_{3}
$$

uniformly for all $\mu_{ \pm} \in I, x_{0} \in S$, where $h=h\left(\mu_{-}, \mu_{+}, e\right)$. Under the assumptions, there exists a null function $z_{0}(\cdot)$ such that

$$
\left|\nabla U^{ \pm}\left(\mu_{ \pm} ; x\right)-\nabla U^{ \pm}\left(\mu_{ \pm} ; x_{0}\right)\right| \leq z_{0}\left(\left|x-x_{0}\right|\right)
$$

for all $x_{0} \in S, \mu_{ \pm} \in I$, and $x \in S \cup \Omega\left(S, S_{1 / 2}^{ \pm}\left(\mu_{ \pm}\right)\right)$, where we define $S_{\varepsilon}^{ \pm}\left(\mu_{ \pm}\right):=\Phi_{\varepsilon}^{ \pm}\left(S, S^{ \pm}\left(\mu_{ \pm}\right)\right)$for all $\varepsilon \in(0,1)$. This follows from the estimate given in [14, Thm. 8.33]. If $d\left(x_{0}, S_{\varepsilon}^{ \pm}\left(\mu_{ \pm}\right)\right)=\left|x_{0}-x_{\varepsilon}^{ \pm}\left(\mu_{ \pm}\right)\right|$for $x_{0} \in S$ and $x_{\varepsilon}^{ \pm}\left(\mu_{ \pm}\right) \in S_{\varepsilon}^{ \pm}\left(\mu_{ \pm}\right)$, then

$$
\varepsilon=U^{ \pm}\left(\mu_{ \pm} ; x_{\varepsilon}^{ \pm}\left(\mu_{ \pm}\right)\right)=\nabla U^{ \pm}\left(\mu_{ \pm} ; x^{*}\right) \cdot\left(x_{\varepsilon}^{ \pm}\left(\mu_{ \pm}\right)-x_{0}\right)
$$

by the theorem of the mean, where $x^{*}$ denotes some point on the linesegment joining $x_{0}$ to $x_{\varepsilon}^{ \pm}\left(\mu_{ \pm}\right)$. Since the vectors $\left(x_{\varepsilon}^{ \pm}\left(\mu_{ \pm}\right)-x_{0}\right)$ and $\nabla U^{ \pm}\left(\mu_{ \pm} ;\right.$ $\left.x_{\varepsilon}^{ \pm}\left(\mu_{ \pm}\right)\right)$are parallel, it follows easily from (8.19) and (8.20) that

$$
\left|\left[\varepsilon / d\left(x_{0}, S_{\varepsilon}^{ \pm}\left(\mu_{ \pm}\right)\right)\right]-\right| \nabla U^{ \pm}\left(\mu_{ \pm} ; x_{0}\right)|| \leq 3 z_{0}\left(d\left[x_{0}, S_{\varepsilon}^{ \pm}\left(\mu_{ \pm}\right)\right]\right),
$$

uniformly for all $x_{0} \in S$ and $\mu_{ \pm} \in I$. Since $d\left(x_{0}, S_{\varepsilon}^{ \pm}\left(\mu_{ \pm}\right)\right) \leq O(\varepsilon)$ as $\varepsilon \downarrow 0$, uniformly for all $x_{0} \in S$ and $\mu_{ \pm} \in I$, it follows from (6.16) and (8.21) that there exists a null function $z_{1}(\cdot)$ such that

$$
\left|\left[d\left(x_{0}, S_{\varepsilon}^{ \pm}\left(\mu_{ \pm}\right)\right) / \varepsilon\right]-Q^{ \pm}\left(\mu_{ \pm} ; x_{0}\right)\right| \leq z_{1}(\varepsilon),
$$

uniformly for all $x_{0} \in S, \mu_{ \pm} \in I$, and $\varepsilon \in(0,1 / 2]$. It follows from (8.16) and (8.22) that there exist positive constants $0<C_{4} \leq C_{5}$ such that

$$
C_{4} \leq\left[d\left(x_{0}, S_{\varepsilon}^{ \pm}\left(\mu_{ \pm}\right)\right) / \varepsilon\right] \leq C_{5}
$$

uniformly for all $x_{0} \in S, \mu_{ \pm} \in I$, and $\varepsilon \in\left(0, \varepsilon_{0}\right]$, where $\varepsilon_{0}>0$ is sufficiently small. Clearly, the point $x(e, \varepsilon):=\alpha(e, \varepsilon) e \in \Omega\left(S_{\varepsilon}^{-}\left(\mu_{-}\right), S_{\varepsilon}^{+}\left(\mu_{+}\right)\right)$ is defined by the equation

$$
f\left(x(e, \varepsilon),\left[d\left(x(e, \varepsilon), S_{\varepsilon}^{-}\left(\mu_{-}\right)\right) / \varepsilon\right],\left[d\left(x(e, \varepsilon), S_{\varepsilon}^{+}\left(\mu_{+}\right)\right) / \varepsilon\right]\right)=0 .
$$

Also, an argument based on (8.19) shows that as $\varepsilon \downarrow 0$, the direction of the shortest line-segment joining $x(e, \varepsilon)$ to the surface $S_{\varepsilon}^{ \pm}\left(\mu_{ \pm}\right)$approaches the direction of the vector $\nabla U^{ \pm}\left(\mu_{ \pm} ; x_{0}(e)\right)$. It follows from straight-forward 
geometric considerations based on this fact that there exists a null function $z_{2}(\cdot)$ such that

$$
\begin{aligned}
& \left|\left[d\left(x(e, \varepsilon), S_{\varepsilon}^{ \pm}\left(\mu_{ \pm}\right)\right) / \varepsilon\right]-\left[d\left(x_{0}(e), S_{\varepsilon}^{ \pm}\left(\mu_{ \pm}\right)\right) / \varepsilon\right] \pm \theta(e)\left[\left(\alpha(e, \varepsilon)-\alpha_{0}(e)\right) / \varepsilon\right]\right| \\
& \leq z_{2}(\varepsilon)
\end{aligned}
$$

uniformly for $e \in \partial B(0 ; 1), \mu_{ \pm} \in I$, and $\varepsilon \in(0,1 / 2]$. By the triangle inequality, we have

$$
\begin{aligned}
& {\left[d\left(S_{\varepsilon}^{-}\left(\mu_{-}\right), S_{\varepsilon}^{+}\left(\mu_{+}\right)\right) / \varepsilon\right]} \\
& \leq\left[d\left(x(e, \varepsilon), S_{\varepsilon}^{-}\left(\mu_{-}\right)\right) / \varepsilon\right]+\left[d\left(x(e, \varepsilon), S_{\varepsilon}^{+}\left(\mu_{+}\right)\right) / \varepsilon\right] \\
& \leq 2 \max \left\{\left[d\left(y_{0}, S_{\varepsilon}^{-}\left(\mu_{-}\right)\right) / \varepsilon\right]+\left[d\left(y_{0}, S_{\varepsilon}^{+}\left(\mu_{+}\right)\right) / \varepsilon\right]: y_{0} \in S\right\}
\end{aligned}
$$

for any $e \in \partial B(0 ; 1), \mu_{ \pm} \in I$ and $\varepsilon \in(0,1)$. In view of (8.23), it follows from (8.26) that there exist positive constants $0<C_{6} \leq C_{7}$ such that

$$
C_{6} \leq\left[d\left(x(e, \varepsilon), S_{\varepsilon}^{-}\left(\mu_{-}\right)\right) / \varepsilon\right]+\left[d\left(x(e, \varepsilon), S_{\varepsilon}^{+}\left(\mu_{+}\right)\right) / \varepsilon\right] \leq C_{7}
$$

uniformly for $e \in \partial B(0 ; 1), \mu_{ \pm} \in I$ and $\varepsilon \in\left(0, \varepsilon_{0}\right]$. In view of Lemma 3.4 , it follows from (8.24) and (8.27) that there exist uniform constants $0<C_{8} \leq C_{9}$ such that

$$
C_{8} \leq\left[d\left(x(e, \varepsilon), S_{\varepsilon}^{ \pm}\left(\mu_{ \pm}\right)\right) / \varepsilon\right] \leq C_{9}
$$

uniformly for $e \in \partial B(0 ; 1), \mu_{ \pm} \in I$ and $\varepsilon \in\left(0, \varepsilon_{0}\right]$. It follows from (8.22) and (8.25) that

$$
\begin{aligned}
& \left|\left[d\left(x(e, \varepsilon), S_{\varepsilon}^{ \pm}\left(\mu_{ \pm}\right)\right) / \varepsilon\right]-Q^{ \pm}\left(\mu_{ \pm} ; x_{0}(e)\right) \pm \theta(e)\left[\left(\alpha(e, \varepsilon)-\alpha_{0}(e)\right) / \varepsilon\right]\right| \\
& \leq z_{3}(\varepsilon)
\end{aligned}
$$

uniformly for $e \in \partial B(0 ; 1), \mu_{ \pm} \in I$ and $\varepsilon \in\left(0, \varepsilon_{0}\right]$, where $z_{3}(\varepsilon):=z_{1}(\varepsilon)+$ $z_{2}(\varepsilon)$. It follows from (8.28) and (8.29) that there exist constants $0<C_{10} \leq$ $C_{11}$ such that

$$
C_{10} \leq Q^{ \pm}\left(\mu_{ \pm} ; x_{0}(e)\right) \mp \theta(e)\left[\left(\alpha(e, \varepsilon)-\alpha_{0}(e)\right) / \varepsilon\right] \leq C_{11}
$$

uniformly for $e \in \partial B(0 ; 1), \mu_{ \pm} \in I$ and $\varepsilon \in\left(0, \varepsilon_{0}\right]$. It follows from (8.16) and (8.30) that there exist constants $0<C_{12} \leq C_{13}$ such that

$$
C_{12} \leq Q^{ \pm}\left(\mu_{ \pm} ; x_{0}(e)\right) \mp \theta(e) t \leq C_{13}
$$

uniformly for all $e \in \partial B(0 ; 1), \mu_{ \pm} \in I, \varepsilon \in\left(0, \varepsilon_{0}\right]$, and $t$ in the interval between $h(e)$ and $\left[\left(\alpha(e, \varepsilon)-\alpha_{0}(e)\right) / \varepsilon\right]$. In view of (8.28), (8.31) and the assumed continuity of $f$, it follows from (8.24) and (8.29) that there exists a null function $z_{4}(\cdot)$ such that

$$
\left|\phi\left(\mu_{-}, \mu_{+}, e,\left[\left(\alpha\left(\mu_{-}, \mu_{+}, e, \varepsilon\right)-\alpha_{0}(e)\right) / \varepsilon\right]\right)\right| \leq z_{4}(\varepsilon)
$$


uniformly for all $e \in \partial B(0 ; 1), \mu_{ \pm} \in I$, and $\varepsilon \in\left(0, \varepsilon_{0}\right]$, where the function $\phi\left(\mu_{-}, \mu_{+}, e, t\right)$ is defined in (8.14). Now it follows from (8.32) and the theorem of the mean (and the fact that $\phi(h)=0$ ) that

$$
\left|\phi^{\prime}\left(t^{*}\right)\left(\left[\left(\alpha\left(\mu_{-}, \mu_{+}, e, \varepsilon\right)-\alpha_{0}(e)\right) / \varepsilon\right]-h\left(\mu_{-}, \mu_{+}, e\right)\right)\right| \leq z_{4}(\varepsilon),
$$

for some value $t^{*}=t^{*}\left(\mu_{-}, \mu_{+}, e, \varepsilon\right)$ lying in the interval between $h\left(\mu_{-}, \mu_{+}, e\right)$ and $\left(\left(\alpha\left(\mu_{-}, \mu_{+}, e, \varepsilon\right)-\alpha_{0}(e)\right) / \varepsilon\right)$. Since

$$
\phi^{\prime}(t)=\theta(e) g\left(x_{0}, Q^{-}\left(\mu_{-} ; x_{0}\right)+\theta t, Q^{+}\left(\mu_{+} ; x_{0}\right)-\theta t\right),
$$

where $g=\left(f_{p}+\left|f_{q}\right|\right)$, it follows from (8.31) and Assumption (A1) that

$$
\phi^{\prime}(t) \geq C>0
$$

for all $t$ between $h\left(\mu_{-}, \mu_{+}, e\right)$ and $\left[\left(\alpha\left(\mu_{-}, \mu_{+}, e, \varepsilon\right)-\alpha_{0}(e)\right) / \varepsilon\right]$, where $C$ is independent of $\mu_{ \pm} \in I$ and $e \in \partial B(0 ; 1)$. Now the assertion follows easily from (8.33) and (8.34).

8.6. Proof of Theorem 8.1. For any fixed values $\mu_{ \pm}>0$, any fixed unit vector $e$, and any values $r \in\left[0, r_{0}\right]$ and $\varepsilon \in(0,1)$, choose the values $\alpha_{0}=\alpha_{0}(e)>0$ and $\alpha^{ \pm}(r, \varepsilon)=\alpha^{ \pm}(e ; r, \varepsilon)>0$ such that $x_{0}:=\alpha_{0} e \in S$ and

$$
x^{ \pm}(r, \varepsilon):=\alpha^{ \pm}(r, \varepsilon) e \in T_{\varepsilon}\left(S^{-}\left(\mu_{-}^{ \pm r}\right), S, S^{+}\left(\mu_{+}^{ \pm r}\right)\right) .
$$

By Lemma 8.5, there exists a null function $z(\cdot)$ such that

$$
\left|\left[\left(\alpha^{ \pm}(r, \varepsilon)-\alpha_{0}(r)\right) / \varepsilon\right]-h^{ \pm}(r)\right| \leq z(\varepsilon)
$$

for any $\mu_{ \pm} \in I:=\left[\mu_{0}^{q_{0}}, \mu_{1}^{q_{0}}\right] \cap\left[\mu_{1}^{-q_{0}}, \mu_{0}^{-q_{0}}\right]$ (with $\left.q_{0}:=1 / r_{0}\right), e \in \partial B(0 ; 1)$, $r \in\left[0, r_{0}\right]$ and $\varepsilon \in(0,1)$, where the values $h^{ \pm}(r)=h^{ \pm}(e, r)$ uniquely solve the equations

$(8.36)$

$\phi^{ \pm}\left(e, r ; h^{ \pm}(r)\right):=f\left(x_{0}, Q^{-}\left(\mu_{-}^{ \pm r} ; x_{0}\right)+\theta h^{ \pm}(r), Q^{+}\left(\mu_{+}^{ \pm r} ; x_{0}\right)-\theta h^{ \pm}(r)\right)=0$.

Here we define $\theta=\theta(e)=e \cdot \nu$, where $\nu=\nu(e)$ denotes the exterior unit normal to the surface $S$ at the point $x_{0}(e) \in S$. Observe that $h^{ \pm}(0)=0$. By (8.18), there exist uniform constants $0<C_{0} \leq C_{1}$ such that

$$
\begin{aligned}
& C_{0} \leq \psi_{-}^{ \pm}(r):=Q^{-}\left(\mu_{-}^{ \pm r} ; x_{0}\right)+\theta h^{ \pm}(r) \leq C_{1}, \\
& C_{0} \leq \psi_{+}^{ \pm}(r):=Q^{+}\left(\mu_{+}^{ \pm r} ; x_{0}\right)-\theta h^{ \pm}(r) \leq C_{1},
\end{aligned}
$$

uniformly for all $\mu_{ \pm} \in I, e \in \partial B(0 ; 1)$, and $r \in\left[0, r_{0}\right]$. By the theorem of the mean, for any fixed $\mu_{ \pm} \in I$ and $e \in \partial B(0 ; 1)$, we have

$$
\begin{aligned}
0= & \phi^{ \pm}\left(e, r+\delta ; h^{ \pm}(r+\delta)\right)-\phi^{ \pm}\left(e, r ; h^{ \pm}(r)\right) \\
= & f_{p}\left(X^{ \pm}(r, \delta)\right)\left(\psi_{-}^{ \pm}(r+\delta)-\psi_{-}^{ \pm}(r)\right) \\
& +f_{q}\left(X^{ \pm}(r, \delta)\right)\left(\psi_{+}^{ \pm}(r+\delta)-\psi_{+}^{ \pm}(r)\right)
\end{aligned}
$$

for $0 \leq r<r+\delta \leq r_{0}$, where $X^{ \pm}(r, \delta)=\left(x_{0}, p^{ \pm}(r, \delta), q^{ \pm}(r, \delta)\right) \in \mathbb{R}^{N+2}$ denotes a point on the straight line-segment joining the point $\left(x_{0}, \psi_{-}^{ \pm}(r)\right.$, 
$\left.\psi_{+}^{ \pm}(r)\right) \in \mathbb{R}^{N+2}$ to the point $\left(x_{0}, \psi_{-}^{ \pm}(r+\delta), \psi_{+}^{ \pm}(r+\delta)\right) \in \mathbb{R}^{N+2}$. We have $C_{0} \leq p^{ \pm}(r, \delta), q^{ \pm}(r, \delta) \leq C_{1}$ by (8.37), (8.38). It follows from (8.39) that

$$
\begin{aligned}
& \left(\psi_{+}^{ \pm}(r+\delta)-\psi_{+}^{ \pm}(r)\right) \\
& =\left(\left|f_{p}\left(X^{ \pm}(r, \delta)\right)\right| / f_{q}\left(X^{ \pm}(r, \delta)\right)\right)\left(\psi_{-}^{ \pm}(r+\delta)-\psi_{-}^{ \pm}(r)\right),
\end{aligned}
$$

where, by Assumption (A1), there exist constants $0<C_{2} \leq C_{3}$ such that

$$
C_{2} \leq\left(\left|f_{p}\left(X^{ \pm}(r, \delta)\right)\right| / f_{q}\left(X^{ \pm}(r, \delta)\right)\right) \leq C_{3}
$$

uniformly for all $\mu_{ \pm} \in I, e \in \partial B(0 ; 1), r \in\left[0, r_{0}\right)$, and sufficiently small $\delta \in\left(0, r_{0}-r\right]$. Eqs. (8.40) and (8.41) imply

$$
\left(\psi_{+}^{ \pm}(r)-\psi_{+}^{ \pm}(0)\right)=P^{ \pm}(r)\left(\psi_{-}^{ \pm}(r)-\psi_{-}^{ \pm}(0)\right)
$$

for $r \in\left[0, r_{0}\right]$, where $P^{ \pm}(r) \in\left[C_{2}, C_{3}\right]$ for any fixed $\mu_{ \pm} \in I$ and $e \in \partial B(0 ; 1)$. In view of the definitions of $\psi_{+}^{ \pm}(r)$ and $\psi_{-}^{ \pm}(r)$, and the fact that $h^{ \pm}(0)=0$, it follows from (8.42) that

$$
\begin{aligned}
\theta h^{ \pm}(r)= & \left(1-\beta^{ \pm}(r)\right)\left(Q^{-}\left(x_{0}\right)-Q^{-}\left(\mu_{-}^{ \pm r} ; x_{0}\right)\right) \\
& +\beta^{ \pm}(r)\left(Q^{+}\left(\mu_{+}^{ \pm r} ; x_{0}\right)-Q^{+}\left(x_{0}\right)\right)
\end{aligned}
$$

for $r \in\left[0, r_{0}\right]$, where $\beta^{ \pm}(r):=1 /\left(1+P^{ \pm}(r)\right) \in\left[\left(1 /\left(1+C_{3}\right)\right),\left(1 /\left(1+C_{2}\right)\right)\right]$ for any fixed $\mu_{ \pm} \in I$ and $e \in \partial B(0 ; 1)$. For any $\mu_{ \pm} \in I$, it follows from Theorem 4.3 (by substituting $\mu=\mu_{+}^{ \pm r}$ in (4.6) and $\mu=\mu_{-}^{ \pm r}$ in (4.7)) that

$$
\begin{aligned}
& \pm\left(Q^{+}\left(\mu_{+}^{ \pm r} ; x_{0}\right)-Q^{+}\left(x_{0}\right)\right) \leq A\left(\mu_{+}\right) \ln \left(\mu_{+}\right) r \\
& \pm\left(Q^{-}\left(x_{0}\right)-Q^{-}\left(\mu_{-}^{ \pm r} ; x_{0}\right)\right) \leq A\left(\mu_{-}\right) \ln \left(\mu_{-}\right) r
\end{aligned}
$$

both uniformly for all $r \in\left[0, r_{0}\right]$ and $x_{0} \in S$. By substituting (8.44) and (8.45) into (8.43), we conclude that

$$
\pm h^{ \pm}(r) \leq\left(\left(1-\beta^{ \pm}\right) A\left(\mu_{-}\right) \ln \left(\mu_{-}\right)+\beta^{ \pm} A\left(\mu_{+}\right) \ln \left(\mu_{+}\right)\right)(r / \theta),
$$

where, for some constants $B_{0} \in(0,1 / 2)$ and $B_{1}=1-B_{0}$, we have $\beta^{ \pm} \in$ $\left[B_{0}, B_{1}\right]$ independent of the choice of $\mu_{ \pm} \in I$ and $e \in \partial B(0 ; 1)$. It follows that

$$
\pm h^{ \pm}(r) \leq\left((1-B) A\left(\mu_{-}\right) \ln \left(\mu_{-}\right)+B A\left(\mu_{+}\right) \ln \left(\mu_{+}\right)\right)(r / \theta)
$$

for any $\mu_{ \pm} \in I, e \in \partial B(0 ; 1)$, and $r \in\left[0, r_{0}\right]$, where $B=B(\cdot)$ is defined as in (8.5). By substituting (8.47) into (8.35), one sees that

(8.48) $\pm \alpha^{ \pm}(e ; r, \varepsilon)$

$$
\leq \pm \alpha_{0}(e)+\left((1-B) A\left(\mu_{-}\right) \ln \left(\mu_{-}\right)+B A\left(\mu_{+}\right) \ln \left(\mu_{+}\right)\right)(r \varepsilon / \theta)+\varepsilon z(\varepsilon)
$$


uniformly for all $\mu_{ \pm} \in I, e \in \partial B(0 ; 1), r \in\left[0, r_{0}\right]$ and $\varepsilon \in(0,1)$. It follows from (8.48) by exponentiation that

$$
\begin{aligned}
& \pm \alpha^{ \pm}(e ; r, \varepsilon) \\
& \leq \pm \alpha_{0}(e)\left(\left(\mu_{-}\right)^{(1-B) A\left(\mu_{-}\right)}\left(\mu_{+}\right)^{B A\left(\mu_{+}\right)}\right)^{ \pm\left(r \varepsilon / \alpha_{0} \theta\right)} \exp \left( \pm \varepsilon z_{1}(\varepsilon)\right) \\
& \leq \pm \alpha_{0}(e)\left(1 / C_{0}\right)^{ \pm\left(r \varepsilon / \alpha_{0} \theta\right)} \exp \left( \pm \varepsilon z_{1}(\varepsilon)\right) \\
& \leq \pm \alpha_{0}(e) \exp \left(\mp \kappa_{0} r \varepsilon \pm \varepsilon z_{1}(\varepsilon)\right)
\end{aligned}
$$

uniformly for all $\mu_{ \pm} \in I, e \in \partial B(0 ; 1), r \in\left[0, r_{0}\right]$ and $\varepsilon \in(0,1)$, where $\kappa_{0}=\ln \left(C_{0}\right) / \min \left\{\alpha_{0}(e)(e \cdot \nu(e)): e \in \partial B(0 ; 1)\right\}$ and $z_{1}(\cdot)$ is a suitable null function.

8.7. Proof of Corollary 8.2. By redefining $\kappa_{0}>0$ and the null function $z(\cdot)$, the inequalities (8.2) and (8.3) can be rewritten in the form:

$$
\begin{array}{r}
T_{\varepsilon}\left(S^{-}\left(\mu_{-}^{r}\right), S, S^{+}\left(\mu_{+}^{r}\right)\right) \leq \lambda^{\left(\varepsilon\left[z(\varepsilon)-\kappa_{0} r\right]\right)} S \\
T_{\varepsilon}\left(S^{-}\left(\mu_{-}^{-r}\right), S, S^{+}\left(\mu_{+}^{-r}\right)\right) \geq \lambda^{\left(\varepsilon\left[\kappa_{0} r-z(\varepsilon)\right]\right)} S
\end{array}
$$

both valid for all $\lambda \in\left[\Lambda_{0}, \Lambda_{1}\right], r \in\left[0, r_{0}\right], \varepsilon \in(0,1)$, and all values $\mu_{ \pm} \in$ $\left[\mu_{0}^{q_{0}}, \mu_{1}^{q_{0}}\right] \cap\left[\mu_{1}^{-q_{0}}, \mu_{0}^{-q_{0}}\right]$ satisfying (8.4) and (8.5). For all ordered triples $\left(\widehat{S}^{-}, \widehat{S}, \widehat{S}^{+}\right) \in \mathbf{X}_{3}\left(\Re_{0}, \Re_{1}\right)$ satisfying (8.7), Lemma $2.5(\mathrm{a})$, (c) implies that

$$
\begin{aligned}
& \lambda^{-r} T_{\varepsilon}\left(S^{-}\left(\mu_{-}^{-r}\right), S, S^{+}\left(\mu_{+}^{-r}\right)\right) \\
& \leq T_{\varepsilon}\left(\lambda^{-r} S^{-}\left(\mu_{-}^{-r}\right), \lambda^{-r} S, \lambda^{-r} S^{+}\left(\mu_{+}^{-r}\right)\right) \\
& \leq T_{\varepsilon}\left(\widehat{S}^{-}, \widehat{S}, \widehat{S}^{+}\right) \leq T_{\varepsilon}\left(\lambda^{r} S^{-}\left(\mu_{-}^{r}\right), \lambda^{r} S, \lambda^{r} S^{+}\left(\mu_{+}^{r}\right)\right) \\
& \leq \lambda^{r} T_{\varepsilon}\left(S^{-}\left(\mu_{-}^{r}\right), S, S^{+}\left(\mu_{+}^{r}\right)\right)
\end{aligned}
$$

for all $\lambda>1, r \geq 0, \varepsilon \in(0,1)$, and $\mu_{ \pm}>0$. Now the assertion follows by substituting (8.49) and (8.50) into (8.51).

8.8. Proof of Theorem 8.4. For fixed $\left(\lambda_{-}, \lambda, \lambda_{+}\right) \in \mathfrak{M}$, and for any $r \in\left[0, r_{0}\right]$, let the ordered triples $\left(S_{-}^{-}(r), S_{-}(r), S_{-}^{+}(r)\right) \in \mathbf{X}_{3}^{-}\left(\Re_{0}, \Re_{1} ; \rho\right)$ and $\left(S_{+}^{-}(r), S_{+}(r), S_{+}^{+}(r)\right) \in \mathbf{X}_{3}^{+}\left(\Re_{0}, \Re_{1} ; \rho\right)$ satisfy (8.11) and (8.12), respectively. It follows that

$$
\begin{array}{ll}
S_{-}(r) \geq \lambda^{-r} S ; & S_{-}^{ \pm}(r) \geq \lambda_{ \pm}^{-r} S^{ \pm}, \\
S_{+}(r) \leq \lambda^{r} S ; & S_{+}^{ \pm}(r) \leq \lambda_{ \pm}^{r} S^{ \pm} .
\end{array}
$$


In view of (8.52) and (8.53), it follows from (8.11) and (8.12) that

$$
\begin{aligned}
S_{-}^{-}(r) & \geq \min \left(S_{-}^{-}(r), P_{+} \lambda^{-r} S\right) \\
& \geq \min \left(\lambda_{-}^{-r} S^{-}, P_{+} \lambda^{-r} S\right)=\lambda^{-r} \Sigma_{-}^{-}\left(\mu_{-}^{-r}\right), \\
S_{-}^{+}(r) & :=\max \left(\lambda_{+}^{-r} S^{+}, \widehat{P}_{-} S_{-}(r)\right) \\
& \geq \max \left(\lambda_{+}^{-r} S^{+}, \widehat{P}_{-} \lambda^{-r} S\right)=\lambda^{-r} \Sigma_{-}^{+}\left(\mu_{+}^{-r}\right), \\
S_{+}^{-}(r) & :=\min \left(\lambda_{-}^{r} S^{-}, \widehat{P}_{+} S_{+}(r)\right) \\
& \leq \min \left(\lambda_{-}^{r} S^{-}, \widehat{P}_{+} \lambda^{r} S\right)=\lambda^{r} \Sigma_{+}^{-}\left(\mu_{-}^{r}\right), \\
S_{+}^{+}(r) & \leq \max \left(S_{+}^{+}(r), P_{-} \lambda^{r} S\right) \\
& \leq \max \left(\lambda_{+}^{r} S^{+}, P_{-} \lambda^{r} S\right)=\lambda^{r} \Sigma_{+}^{+}\left(\mu_{+}^{r}\right),
\end{aligned}
$$

all for any $r \in\left[0, r_{0}\right]$, where we define

$$
\begin{array}{ll}
\Sigma_{-}^{-}(\mu)=\min \left(\mu S^{-}, P_{+} S\right) ; & \Sigma_{-}^{+}(\mu)=\max \left(\mu S^{+}, \widehat{P}_{-} S\right), \\
\Sigma_{+}^{-}(\mu)=\min \left(\mu S^{-}, \widehat{P}_{+} S\right) ; \quad \Sigma_{+}^{+}(\mu)=\max \left(\mu S^{+}, P_{-} S\right),
\end{array}
$$

all for any $\mu>0$. In view of the inequalities (8.52)-(8.57), it follows from Lemma 2.5 and Corollary 8.2 that there exist a value $\kappa_{0}>0$ and a null function $z(\cdot)$ such that

$$
\begin{aligned}
& \lambda^{\left(\kappa_{0} r-z(\varepsilon)\right) \varepsilon}\left(\lambda^{-r} S\right) \\
& \leq T_{\varepsilon}\left(\lambda^{-r} \Sigma_{-}^{-}\left(\mu_{-}^{-r}\right), \lambda^{-r} S, \lambda^{-r} \Sigma_{-}^{+}\left(\mu_{+}^{-r}\right)\right) \\
& \leq T_{\varepsilon}\left(S_{-}^{-}(r), S_{-}(r), S_{-}^{+}(r)\right) \leq T_{\varepsilon}\left(\widehat{S}^{-}, \widehat{S}, \widehat{S}^{+}\right) \\
& \leq T_{\varepsilon}\left(S_{+}^{-}(r), S_{+}(r), S_{+}^{+}(r)\right) \\
& \leq T_{\varepsilon}\left(\lambda^{r} \Sigma_{+}^{-}\left(\mu_{-}^{r}\right), \lambda^{r} S, \lambda^{r} \Sigma_{+}^{+}\left(\mu_{+}^{r}\right)\right) \\
& \leq \lambda^{\left(z(\varepsilon)-\kappa_{0} r\right) \varepsilon}\left(\lambda^{r} S\right)
\end{aligned}
$$

uniformly for all $\left(\lambda_{-}, \lambda, \lambda_{+}\right) \in \mathfrak{M}$, all $\varepsilon \in(0,1)$, all $r \in\left[0, r_{0}\right]$, all ordered triples $\left(S_{-}^{-}(r), S_{-}(r), S_{-}^{+}(r)\right) \in \mathbb{X}_{3}^{-}\left(\Re_{0}, \Re_{1} ; \rho\right)$ and $\left(S_{+}^{-}(r), S_{+}(r), S_{+}^{+}(r)\right) \in$ $\mathbf{x}_{3}^{+}\left(\Re_{0}, \Re_{1} ; \rho\right)$ satisfying (8.11) and (8.12), respectively, for a particular $\left(\lambda_{-}, \lambda, \lambda_{+}\right)$, and all ordered triples $\left(\widehat{S}^{-}, \widehat{S}, \widehat{S}^{+}\right) \in \mathbf{X}_{3}\left(\Re_{0}, \Re_{1}\right)$ satisfying (8.13). It is also clear from (8.11) and (8.12) that

$$
\begin{array}{ll}
S_{-}(r) \geq P_{-} S_{-}^{-}(r) ; & S_{-}^{+}(r) \geq \widehat{P}_{-} S_{-}(r) \geq P_{-} \widehat{P}_{-} S_{-}^{-}(r), \\
S_{+}(r) \leq P_{+} S_{+}^{+}(r) ; & S_{+}^{-}(r) \leq \widehat{P}_{+} S_{+}(r) \leq P_{+} \widehat{P}_{+} S_{+}^{+}(r),
\end{array}
$$


all for any $r \in\left[0, r_{0}\right]$. Therefore, we have

$$
\begin{aligned}
& P_{-} S_{-}^{-}(r) \leq S_{-}(r) \leq \widehat{S} \leq S_{+}(r) \leq P_{+} S_{+}^{+}(r), \\
& P_{-} \widehat{P}_{-} S_{-}^{-}(r) \leq S_{-}^{+}(r) \leq \widehat{S}^{+} \leq S_{+}^{+}(r), \\
& S_{-}^{-}(r) \leq \widehat{S}^{-} \leq S_{+}^{-}(r) \leq P_{+} \widehat{P}_{+} S_{+}^{+}(r),
\end{aligned}
$$

for any $r \in\left[0, r_{0}\right]$ and any $\left(\widehat{S}^{-}, \widehat{S}, \widehat{S}^{+}\right) \in \mathbf{X}_{3}\left(\Re_{0}, \Re_{1}\right)$ satisfying (8.13). In view of assumptions (8.8) and (8.9), it follows from (8.59)-(8.61) and Lemma 2.5 (a) that

$$
\lambda^{C \varepsilon}\left(P_{-} S_{-}^{-}(r)\right) \leq T_{\varepsilon}\left(\widehat{S}^{-}, \widehat{S}, \widehat{S}^{+}\right) \leq \lambda^{-C \varepsilon}\left(P_{+} S_{+}^{+}(r)\right)
$$

for all $r \in\left[0, r_{0}\right], \lambda \in\left[\Lambda_{0}, \Lambda_{1}\right]$, and $\varepsilon \in(0,1)$, and for any $\left(\widehat{S}^{-}, \widehat{S}, \widehat{S}^{+}\right) \in$ $\mathbf{X}_{3}\left(\Re_{0}, \Re_{1}\right)$ satisfying (8.13). It follows from (8.58) and (8.62) that

$$
\begin{aligned}
\phi_{-}(r, \varepsilon) S_{-}(r) & =\phi_{-}(r, \varepsilon) \max \left(\lambda^{-r} S, P_{-} S_{-}^{-}(r)\right) \leq T_{\varepsilon}\left(\widehat{S}^{-}, \widehat{S}, \widehat{S}^{+}\right) \\
& \leq \phi_{+}(r, \varepsilon) \min \left(\lambda^{r} S, P_{+} S_{+}^{+}(r)\right)=\phi_{+}(r, \varepsilon) S_{+}(r),
\end{aligned}
$$

uniformly for all $\left(\lambda_{-}, \lambda, \lambda_{+}\right) \in \mathfrak{M}$, all $\varepsilon \in(0,1)$, all $r \in\left[0, r_{0}\right]$, all ordered triples $\left(S_{-}^{-}(r), S_{-}(r), S_{-}^{+}(r)\right) \in \mathbf{X}_{3}^{-}\left(\Re_{0}, \Re_{1} ; \rho\right)$ and $\left(S_{+}^{-}(r), S_{+}(r), S_{+}^{+}(r)\right) \in$ $\mathbf{X}_{3}^{+}\left(\Re_{0}, \Re_{1} ; \rho\right)$ satisfying (8.11) and (8.12), respectively, for any particular $\left(\lambda_{-}, \lambda, \lambda_{+}\right) \in \mathfrak{M}$, and all ordered triples $\left(\widehat{S}^{-}, \widehat{S}, \widehat{S}^{+}\right) \in \mathbf{X}_{3}\left(\Re_{0}, \Re_{1}\right)$ satisfying (8.13), where we define

$$
\begin{aligned}
& \phi_{-}(r, \varepsilon)=\min \left\{\lambda^{C \varepsilon}, \lambda^{\left(\kappa_{0} r-z(\varepsilon)\right) \varepsilon}\right\} ; \\
& \phi_{+}(r, \varepsilon)=\max \left\{\lambda^{-C \varepsilon}, \lambda^{\left(z(\varepsilon)-\kappa_{0} r\right) \varepsilon}\right\} .
\end{aligned}
$$

Now the assertion (8.10) follows directly from (8.63) and (8.64), where we decrease the constant $\kappa_{0}>0$ if necessary (so that $\kappa_{0} r_{0} \leq C$ ).

\section{Multi-surface problem: Convergence of successive approximations.}

9.1. Assumptions, definitions, notation. The assumptions of $\S 7.1$ apply throughout this section. For any constants $0<P_{*}^{+}<1<P_{*}^{-}$, let $\mathbb{Y}\left(P_{*}^{-}, P_{*}^{+}\right)$denote the family of all multisurfaces $\boldsymbol{S}=\left(S_{1}, \ldots, S_{k}\right) \in$ $\mathbf{X}_{k}\left(\Re_{0}, \Re_{1}\right)$ such that $P_{*}^{-} S_{*}^{-}<S_{i}<P_{*}^{+} S_{*}^{+}$for $i=1, \ldots, k$.

Theorem 9.2. In Problem 1.1 (under Assumptions 7.1 ), let $\widetilde{\boldsymbol{S}}=\left(\widetilde{S}_{1}, \ldots\right.$, $\left.\widetilde{S}_{k}\right)$ denote a classical solution such that $\widetilde{\boldsymbol{S}} \in \mathbb{Y} \cap \mathbf{X}_{k}\left(\Re_{0}, \Re_{1} ; \rho_{1}\right)$ for some $\rho_{1} \in\left(0, \rho_{0}\right]$. Then (1.10) holds, where $\widetilde{\boldsymbol{S}}_{\varepsilon} \in \mathbb{Y}$ denotes the unique solution of Problem 1.3 at each $\varepsilon \in(0,1)$.

Theorem 9.3. In Problem 1.1 (under Assumptions 7.1), let $\widetilde{\boldsymbol{S}}=\left(\widetilde{S}_{1}, \ldots\right.$, $\left.\widetilde{S}_{k}\right)$ denote a classical solution such that $\widetilde{\boldsymbol{S}} \in \mathbb{Y} \cap \mathbf{x}_{k}\left(\Re_{0}, \Re_{1} ; \rho_{1}\right)$ for some 
$\rho_{1} \in\left(0, \rho_{0}\right]$. Given an initial multisurface $\boldsymbol{S}_{0} \in \mathbb{Y}$, let the sequence of multisurfaces $\boldsymbol{S}_{n} \in \mathbb{Y}, n \in \mathbb{N}$, be defined inductively by Eq. (1.8), where $\left(\varepsilon_{n}\right)_{n=0}^{\infty}$ denotes any given null sequence of values in the interval $(0,1)$ such that $\Sigma_{n=0}^{\infty} \varepsilon_{n}=\infty$. Then $\boldsymbol{S}_{n} \rightarrow \widetilde{\boldsymbol{S}}$ as $n \rightarrow \infty$, in the sense of Eq. (1.11).

As we will show (see $\S \S 9.7,9.8$ ), Theorems 9.2 and 9.3 are quite direct consequences of Theorems 9.4 and 9.5, which follow.

Theorem 9.4. In Problem 1.1 (under Assumptions 7.1 ), let $\widetilde{\boldsymbol{S}}=\left(\widetilde{S}_{1}, \ldots\right.$, $\left.\widetilde{S}_{k}\right)$ denote a classical solution such that $\widetilde{\boldsymbol{S}} \in \mathbf{Y}\left(P_{*}^{-}, P_{*}^{+}\right) \cap \mathbf{X}_{k}\left(\Re_{0}, \Re_{1} ; \rho_{1}\right)$ for some fixed values $\rho_{1} \in\left(0, \rho_{0}\right]$ and $0<P_{*}^{+}<1<P_{*}^{-}$. For any $r \geq 0$, let the multisurfaces $\boldsymbol{S}^{ \pm}(r)=\left(S_{1}^{ \pm}(r), \ldots, S_{k}^{ \pm}(r)\right) \in \mathbb{Y}$ be defined inductively such that

$$
\begin{aligned}
S_{i}^{-}(r) & :=\max \left(\lambda_{i}^{-r} \widetilde{S}_{i}, P_{i}^{-} S_{i-1}^{-}(r)\right) ; \\
S_{i}^{+}(r) & :=\min \left(\lambda_{i}^{r} \widetilde{S}_{i}, P_{i}^{+} S_{i+1}^{+}(r)\right),
\end{aligned}
$$

both for all $i=1, \ldots, k$, where we define $S_{0}^{-}(r):=S_{*}^{-}$and $S_{k+1}^{+}(r):=S_{*}^{+}$, and where the fixed values $P_{1}^{-}, P_{2}^{-}, \ldots, P_{k}^{-}>1, P_{1}^{+}, P_{2}^{+}, \ldots, P_{k}^{+} \in(0,1)$, and $\lambda_{1}, \ldots, \lambda_{k}>1$ are all chosen appropriately (as discussed in the proof in $§ 9.9)$. Then:

(a) For any $0 \leq \alpha \leq \beta, r \geq 0, i=1, \ldots, k$, and $t \in\left[0, r_{1}\right]$ (where $r_{1}>0$ is sufficiently small), we have

$$
\begin{gathered}
\boldsymbol{S}^{-}(\beta) \leq \boldsymbol{S}^{-}(\alpha) \leq \boldsymbol{S}^{-}(0)=\widetilde{\boldsymbol{S}}=\boldsymbol{S}^{+}(0) \leq \boldsymbol{S}^{+}(\alpha) \leq \boldsymbol{S}^{+}(\beta) \\
\lambda_{i}^{-r} \widetilde{S}_{i} \leq S_{i}^{-}(r) \leq \widetilde{S}_{i} \leq S_{i}^{+}(r) \leq \lambda_{i}^{r} \widetilde{S}_{i} \\
S_{i}^{ \pm}(t)=\lambda_{i}^{ \pm t} \widetilde{S}_{i}
\end{gathered}
$$

Due to (9.3), we have

$$
\boldsymbol{M}_{\boldsymbol{\lambda}}(\boldsymbol{S}, \widetilde{\boldsymbol{S}}) \leq \inf \left\{r \geq 0: \boldsymbol{S}^{-}(r) \leq \boldsymbol{S} \leq \boldsymbol{S}^{+}(r)\right\}
$$

for any multisurface $\boldsymbol{S} \in \mathbb{Y}$.

(b) Let the value $r_{0}>0$ be suitably chosen. Then $\boldsymbol{S}^{ \pm}(r)=\boldsymbol{S}^{ \pm}\left(r_{0}\right)$ for all $r \geq r_{0}$. We also have that $\boldsymbol{T}_{\varepsilon}(\widehat{\mathbb{Y}}) \subset \widehat{\mathbb{Y}}$ for any $\varepsilon \in(0,1)$, where we define $\widehat{\mathbb{Y}}=\left\{\boldsymbol{S} \in \mathbb{Y}: \boldsymbol{S}^{-}\left(r_{0}\right) \leq \boldsymbol{S} \leq \boldsymbol{S}^{+}\left(r_{0}\right)\right\}$. Moreover, we have $\mathbb{Y}\left(P_{*}^{-}, P_{*}^{+}\right) \subset \widehat{\mathbb{Y}}$.

(c) There exist a value $\kappa_{0}>0$ and a null function $z_{0}(\cdot)$ such that

$$
\lambda_{i}^{\left(\kappa_{0} r-z_{0}(\varepsilon)\right) \varepsilon} S_{i}^{-}(r) \leq T_{\varepsilon, i}(\boldsymbol{S}) \leq \lambda_{i}^{\left(z_{0}(\varepsilon)-\kappa_{0} r\right) \varepsilon} S_{i}^{+}(r)
$$

for all $i=1, \ldots, k, r \in\left[0, r_{0}\right], \varepsilon \in(0,1)$, and multisurfaces $\boldsymbol{S} \in \mathbb{Y}$ such that

$$
\boldsymbol{S}^{-}(r) \leq \boldsymbol{S} \leq \boldsymbol{S}^{+}(r)
$$


Theorem 9.5. In the context of Theorem 9.4, we have

$$
\begin{aligned}
S_{i}^{-}\left(r-C\left(\kappa_{0} r-z_{0}(\varepsilon)\right) \varepsilon\right) & \leq \lambda_{i}^{\left(\kappa_{0} r-z_{0}(\varepsilon)\right) \varepsilon} S_{i}^{-}(r), \\
\lambda_{i}^{\left(z_{0}(\varepsilon)-\kappa_{0} r\right) \varepsilon} S_{i}^{+}(r) & \leq S_{i}^{+}\left(r-C\left(\kappa_{0} r-z_{0}(\varepsilon)\right) \varepsilon\right)
\end{aligned}
$$

for any $i=1, \ldots, k$, and for any $r \in\left[0, r_{0}\right]$ and $\varepsilon \in(0,1)$ such that $z_{0}(\varepsilon)<$ $\kappa_{0} r$. Here we define $C=\left(\min \left\{\ln \left(\lambda_{i}\right)\right\}\right) /\left(\max \left\{\ln \left(\lambda_{i}\right)\right\}\right) \in(0,1)$. In view of (9.6), it follows that

$$
\begin{aligned}
& \boldsymbol{S}^{-}\left(\left(1-C \kappa_{0} \varepsilon\right) r+C \varepsilon z_{0}(\varepsilon)\right) \\
& \leq \boldsymbol{T}_{\varepsilon}(\boldsymbol{S}) \leq \boldsymbol{S}^{+}\left(\left(1-C \kappa_{0} \varepsilon\right) r+C \varepsilon z_{0}(\varepsilon)\right)
\end{aligned}
$$

for any $r \in\left[0, r_{0}\right]$ and $\varepsilon \in(0,1)$ such that $z_{0}(\varepsilon)<\kappa_{0} r$, and for any multisurface $\boldsymbol{S} \in \mathbb{Y}$ satisfying (9.7).

9.6. Remark. The inequalities (9.8) and (9.9) hold for all $i=1, \ldots, k$, $r \in\left[0, r_{1}\right]$ and $\varepsilon \in\left(0, \varepsilon_{0}\right]$, provided that $C=1$ and the values $r_{1} \in\left(0, r_{0}\right]$ and $\varepsilon_{0} \in(0,1)$ are sufficiently small. In view of $(9.4)$, it follows that if $C=1$, and if the values $r_{1} \in\left(0, r_{0}\right], \varepsilon_{0} \in(0,1)$ are sufficiently small, then (9.10) holds for any $r \in\left[0, r_{1}\right]$ and $\varepsilon \in\left(0, \varepsilon_{0}\right]$, and for any $\boldsymbol{S} \in \mathbb{Y}$ satisfying (9.7).

9.7. Proof of Theorem 9.2. By Lemma 7.7, there exist values $0<$ $P_{*}^{+}<1<P_{*}^{-}$such that $\widetilde{\boldsymbol{S}}_{\varepsilon} \in \mathbb{Y}\left(P_{*}^{-}, P_{*}^{+}\right)$for all $\varepsilon \in(0,1)$, where $\widetilde{\boldsymbol{S}}_{\varepsilon}$ solves Problem 1.3 at $\varepsilon$. In the context of Theorem 9.4, we define $r(\varepsilon):=\min \{r \in$ $\left.[0, \infty): \boldsymbol{S}^{-}(r) \leq \widetilde{\boldsymbol{S}}_{\varepsilon} \leq \boldsymbol{S}^{+}(r)\right\} \in\left[0, r_{0}\right]$ for all $\varepsilon \in(0,1)$. Then

$$
\begin{aligned}
& \boldsymbol{S}^{-}\left(\left(1-C \kappa_{0} \varepsilon\right) r(\varepsilon)+C \varepsilon z_{0}(\varepsilon)\right) \\
& \leq \boldsymbol{T}_{\varepsilon}\left(\widetilde{\boldsymbol{S}}_{\varepsilon}\right) \leq \boldsymbol{S}^{+}\left(\left(1-C \kappa_{0} \varepsilon\right) r(\varepsilon)+C \varepsilon z_{0}(\varepsilon)\right)
\end{aligned}
$$

by Theorem 9.5 (Eq. (9.10)). Since $\widetilde{\boldsymbol{S}}_{\varepsilon}=\boldsymbol{T}_{\varepsilon}\left(\widetilde{\boldsymbol{S}}_{\varepsilon}\right)$, it follows from the definition of $r(\varepsilon)$ that $r(\varepsilon) \leq\left(1-C \kappa_{0} \varepsilon\right) r(\varepsilon)+C \varepsilon z_{0}(\varepsilon)$. Therefore, we have $r(\varepsilon) \leq\left(z_{0}(\varepsilon) / \kappa_{0}\right)$ for each $\varepsilon \in(0,1)$. Thus $\boldsymbol{M}_{\boldsymbol{\lambda}}\left(\widetilde{\boldsymbol{S}}, \widetilde{\boldsymbol{S}}_{\varepsilon}\right) \leq z_{0}(\varepsilon) / \kappa_{0}$ by $(9.5)$, and the assertion follows from the equivalence of the metrics $\boldsymbol{M}$ and $\boldsymbol{M}_{\boldsymbol{\lambda}}$.

9.8. Proof of Theorem 9.3. For a given initial multisurface $\boldsymbol{S}_{0} \in \mathbb{Y}$, choose values $0<P_{*}^{+}<1<P_{*}^{-}$such that $\widetilde{\boldsymbol{S}}, \boldsymbol{S}_{0} \in \mathbb{Y}\left(P_{*}^{-}, P_{*}^{+}\right)$. Then $\boldsymbol{S}^{-}\left(r_{0}\right) \leq \boldsymbol{S}_{n} \leq \boldsymbol{S}^{+}\left(r_{0}\right)$ for all $n \in \mathbb{N}$, by Theorem 9.4(b). For each $n=$ $0,1,2, \ldots$, let $E(n) \in\left[0, r_{0}\right]$, denote the minimum value of $r \in\left[0, r_{0}\right]$ such that $\boldsymbol{S}^{-}(r) \leq \boldsymbol{S}_{n} \leq \boldsymbol{S}^{+}(r)$. By Theorem 9.5 (Eq. (9.10)), we have

$$
E(n+1) \leq\left(1-C \kappa_{0} \varepsilon_{n}\right) E(n)+C \varepsilon_{n} z_{0}\left(\varepsilon_{n}\right)
$$

for any $n=0,1,2, \ldots$ such that $z_{0}\left(\varepsilon_{n}\right)<\kappa_{0} E(n)$. For any given $\eta \in\left(0, r_{0}\right]$, there exists a positive integer $n_{0}$ such that if $n \geq n_{0}$, then $z_{0}\left(\varepsilon_{n}\right)<\left(\kappa_{0} \eta / 4\right)$ 
and $\varepsilon_{n} z_{0}\left(\varepsilon_{n}\right)<(\eta / 2)$. It follows from (9.11) that

$$
\begin{aligned}
E(n+1) & \leq E(n)-C\left(\kappa_{0} E(n)-z_{0}\left(\varepsilon_{n}\right)\right) \varepsilon_{n} \\
& \leq E(n)-C\left(\left(\kappa_{0} \eta / 2\right)-z_{0}\left(\varepsilon_{n}\right)\right) \varepsilon_{n} \\
& \leq E(n)-\left(\kappa_{0} \eta / 4\right) \varepsilon_{n}
\end{aligned}
$$

for $n \geq n_{0}$ and $E(n) \in\left[(\eta / 2), r_{0}\right]$, whereas

$$
E(n+1) \leq E(n)+\varepsilon_{n} z_{0}\left(\varepsilon_{n}\right) \leq E(n)+(\eta / 2)
$$

whenever $n \geq n_{0}$. Since $\Sigma_{n=0}^{\infty} \varepsilon_{n}=\infty$, it follows directly from (9.12) that $E\left(n_{1}\right) \leq(\eta / 2)$ for some integer $n_{1} \geq n_{0}$. It then follows from (9.12) and (9.13) that $E(n) \leq \eta$ for all $n \geq n_{1}$. Since $\eta \in\left(0, r_{0}\right]$ was arbitrary in the preceding argument, it follows that $E(n) \rightarrow 0$ as $n \rightarrow \infty$. Therefore, $\boldsymbol{M}_{\boldsymbol{\lambda}}\left(\boldsymbol{S}_{n}, \widetilde{\boldsymbol{S}}\right) \rightarrow 0$ as $n \rightarrow \infty$, by (9.5). The assertion (1.11) now follows, since the metrics $\boldsymbol{M}$ and $\boldsymbol{M}_{\boldsymbol{\lambda}}$ are equivalent.

9.9. Proof of Theorem 9.4. Assume that either $k=2 m-1$ or $k=2 m$, where $m$ is a fixed positive integer. Choose the values $\Lambda_{0}=2, \Lambda_{1}=2^{m+1}$, and $r_{0}=2 \ln \left(\Re_{1} / \Re_{0}\right) / \ln (2)>0$. For convenience, we set $\widetilde{S}_{0}:=S_{*}^{-}$and $\widetilde{S}_{k+1}:=S_{*}^{+}$. Let $\rho_{2}=\min \left\{P_{*}^{+},\left(1 / \Lambda_{1}\right)^{k r_{0}}\right\} \rho_{1}>0$, where $\widetilde{\boldsymbol{S}} \in \mathbb{Y}\left(P_{*}^{-}, P_{*}^{+}\right) \cap$ $\mathbf{X}_{k}\left(\Re_{0}, \Re_{1} ; \rho_{1}\right)$. Let $\widehat{h} \in(0,1 / 2]$ denote a fixed value such that

$$
\begin{gathered}
(1-\widehat{h})^{k}>\max \left\{P_{*}^{+},\left(1 / P_{*}^{-}\right)\right\} \\
\widetilde{S}_{i}<(1-\widehat{h})^{2} \widetilde{S}_{i+1} \text { for } i=0, \ldots, k .
\end{gathered}
$$

Let $h:=\min \left\{\widehat{h} ; \eta_{1}, \eta_{2}, \ldots, \eta_{k}\right\}$ and let $g_{0}(t):=\min \left\{g_{0,1}(t), \ldots, g_{0, k}(t)\right\}>0$ for each $t \in(0, h]$, where, for each $i=1, \ldots, k, \eta_{i} \in(0,1 / 2]$ and $g_{0, i}(\cdot):\left(0, \eta_{i}\right]$ $\rightarrow \mathbb{R}_{+}$denote the constant and the positive null function of Remark 3.2, in the case where $r=\rho_{2}$ and $T_{\varepsilon}:=T_{\varepsilon, i}$ (see $\left.\S 2.6\right)$. Let the fixed $(k+1)$-tuples $\left(P_{0}^{+}, \ldots, P_{k}^{+}\right),\left(P_{1}^{-}, \ldots, P_{k+1}^{-}\right)$be defined by $P_{i}^{ \pm}:=1 \mp \delta_{i}^{ \pm}$, where the fixed $(k+1)$-tuples $\left(\delta_{0}^{+}, \ldots, \delta_{k}^{+}\right),\left(\delta_{1}^{-}, \ldots, \delta_{k+1}^{-}\right)$are defined inductively such that $\delta_{k+1}^{-}, \delta_{0}^{+} \in(0, h]$, and such that

$$
\delta_{i}^{ \pm} \in(0, h], \quad \delta_{i}^{-} \in\left(0, g_{0}\left(\delta_{i+1}^{-}\right)\right], \quad \delta_{i}^{+} \in\left(0, g_{0}\left(\delta_{i-1}^{+}\right)\right],
$$

for all $i=1, \ldots, k$. Choose $C:=\min \left\{g_{0}\left(\delta_{1}^{+}\right), \ldots, g_{0}\left(\delta_{k}^{+}\right), g_{0}\left(\delta_{1}^{-}\right), \ldots, g_{0}\left(\delta_{k}^{-}\right)\right\}$ $>0$. By Theorem 3.1 and Remark 3.2, we have

$$
T_{\varepsilon, i}\left(S, P_{i}^{-} S, P_{i}^{-} P_{i+1}^{-} S\right) \geq \lambda^{C \varepsilon} P_{i}^{-} S
$$

for all $i=1, \ldots, k, S \in \mathbb{X}^{-}\left(\Re_{0}, \Re_{1} ; \rho_{2}\right), \varepsilon \in(0,1)$, and $\lambda \in\left[\Lambda_{0}, \Lambda_{1}\right]$, whereas

$$
T_{\varepsilon, i}\left(P_{i-1}^{+} P_{i}^{+} S, P_{i}^{+} S, S\right) \leq \lambda^{-C \varepsilon} P_{i}^{+} S
$$

for all $i=1, \ldots, k, S \in \mathbb{X}^{+}\left(\Re_{0}, \Re_{1} ; \rho_{2}\right), \varepsilon \in(0,1)$, and $\lambda \in\left[\Lambda_{0}, \Lambda_{1}\right]$. 
Given the above $(k+1)$-tuples $\left(P_{0}^{+}, \ldots, P_{k}^{+}\right),\left(P_{1}^{-}, \ldots, P_{k+1}^{-}\right)$, we will show that parts (a) and (b) hold for any particular choice of $\boldsymbol{\lambda}=\left(\lambda_{1}, \ldots, \lambda_{k}\right)$ $\in\left[\Lambda_{0}, \Lambda_{1}\right]^{k}$. For each $r \geq 0$, let the surfaces $S_{0}^{-}(r), S_{1}^{-}(r), \ldots, S_{k}^{-}(r), S_{k+1}^{-}(r)$ be defined inductively by (9.1a) (starting with $S_{0}^{-}(r):=\widetilde{S}_{0}$ ), and let the surfaces $S_{0}^{+}(r), S_{1}^{+}(r), \ldots, S_{k}^{+}(r), S_{k+1}^{+}(r)$ be defined inductively by (9.1b) (starting with $\left.S_{k+1}^{+}(r):=\widetilde{S}_{k+1}\right)$, where we set $\lambda_{0}=1=\lambda_{k+1}$. Since $h \in$ $(0, \widehat{h}]$, it follows from $(9.15)$ and $(9.16)$ that

$$
\begin{aligned}
\lambda_{i}^{-r} \widetilde{S}_{i}>\lambda_{i}^{-r}(1+\widehat{h})^{2} \widetilde{S}_{i-1} & \geq(1+\widehat{h}) \widetilde{S}_{i-1} \geq P_{i}^{-} \widetilde{S}_{i-1} \\
\lambda_{i}^{r} \widetilde{S}_{i}<\lambda_{i}^{r}(1-\widehat{h})^{2} \widetilde{S}_{i+1} & \leq(1-\widehat{h}) \widetilde{S}_{i+1} \leq P_{i}^{+} \widetilde{S}_{i+1},
\end{aligned}
$$

for $i=1, \ldots, k$ and $r \in\left[0, r_{1}\right]$, where $r_{1}=\ln (1+\widehat{h}) /(m+1) \ln (2)$. For given $i \in\{1, \ldots, k\}$, it follows from (9.1a) and (9.19) (resp. (9.1b) and (9.20)) that $S_{i}^{-}(r)=\lambda_{i}^{-r} \widetilde{S}_{i}\left(\operatorname{resp} . S_{i}^{+}(r)=\lambda_{i}^{r} \widetilde{S}_{i}\right)$, provided that $S_{i-1}^{-}(r)=\lambda_{i-1}^{-r} \widetilde{S}_{i-1}$ (resp. $\left.S_{i+1}^{+}(r)=\lambda_{i+1}^{r} \widetilde{S}_{i+1}\right)$. Since $S_{0}^{-}(r)=\lambda_{0}^{-r} \widetilde{S}_{0}=\widetilde{S}_{0}$ and $S_{k+1}^{+}(r)=$ $\lambda_{k+1}^{r} \widetilde{S}_{k+1}=\widetilde{S}_{k+1}$, it follows by induction that $S_{i}^{ \pm}(r)=\lambda_{i}^{ \pm r} \widetilde{S}_{i}$ for $r \in\left[0, r_{1}\right]$ and $i=1, \ldots, k$, as was asserted in (9.4). As a special case of (9.4), we have that $\boldsymbol{S}^{ \pm}(0)=\widetilde{\boldsymbol{S}}$, as is asserted in (9.2). The remaining assertions in (9.2) and (9.3) follow easily from (9.1) and (9.4). A simple extension of the same arguments shows that

$$
S_{0}^{+}(r) \geq S_{0}^{+}(0)=\widetilde{S}_{0} ; \quad S_{k+1}^{-}(r) \leq S_{k+1}^{-}(0)=\widetilde{S}_{k+1}
$$

for any $r \geq 0$. Turning to part (b), we define $r_{0}=2 \ln \left(\Re_{1} / \Re_{0}\right) / \ln (2)>0$. It follows that $\lambda_{i}^{r} \geq 2^{r} \geq\left(\Re_{1} / \Re_{0}\right)$ for $r \geq r_{0}$ and $i=1, \ldots, k$, and therefore that $\lambda_{i}^{r} \widetilde{S}_{i} \geq \widetilde{S}_{k+1}$ and $\lambda_{i}^{-r} \widetilde{S}_{i} \leq \widetilde{S}_{0}$ for $r \geq r_{0}$ and $i=1, \ldots, k$. Since $\widetilde{S}_{0} \leq S_{i}^{-}(r) \leq S_{i}^{+}(r) \leq \widetilde{S}_{k+1}$ for any $r \geq 0$ and $i=1, \ldots, k$, it follows by $(9.1 \mathrm{a}, \mathrm{b})$ that

$$
S_{i}^{ \pm}(r)=P_{i}^{ \pm} S_{i \pm 1}^{ \pm}(r)
$$

for $r \geq r_{0}$ and $i=1, \ldots, k$. Thus $\boldsymbol{S}^{ \pm}(r)=\boldsymbol{S}^{ \pm}\left(r_{0}\right)$ for $r \geq r_{0}$. By (9.16) and (9.22), we have

$$
S_{i}^{+}\left(r_{0}\right) \geq(1-\widehat{h}) S_{i+1}^{+}\left(r_{0}\right) ; \quad S_{i}^{-}\left(r_{0}\right) \leq(1+\widehat{h}) S_{i-1}^{-}\left(r_{0}\right)
$$

for $i=1, \ldots, k$, and it follows from (9.14) and (9.23) that

$$
\begin{aligned}
S_{i}^{-}\left(r_{0}\right) & \leq(1+\widehat{h})^{k} \widetilde{S}_{0}<P_{*}^{-} \widetilde{S}_{0} \leq S_{i} \\
& \leq P_{*}^{+} \widetilde{S}_{k+1} \leq(1-\widehat{h})^{k} \widetilde{S}_{k+1} \leq S_{i}^{+}\left(r_{0}\right)
\end{aligned}
$$

for $i=1, \ldots, k$, and any multisurface $\boldsymbol{S}=\left(S_{1}, \ldots, S_{k}\right) \in \mathbb{Y}\left(P_{*}^{-}, P_{*}^{+}\right)$. Also, it follows from (9.14), (9.16), and (9.22) that $S_{i}^{ \pm}\left(r_{0}\right) \in \mathbb{X}^{ \pm}\left(\Re_{0}, \Re_{1} ; \rho_{2}\right)$ for 
$i=1, \ldots, k$. In view of this, it follows from (9.17), (9.18), (9.21), (9.22), and Lemma 2.5(a) that

$$
\boldsymbol{S}^{-}\left(r_{0}\right) \leq \boldsymbol{T}_{\varepsilon}\left(\boldsymbol{S}^{-}\left(r_{0}\right)\right) \leq \boldsymbol{T}_{\varepsilon}(\boldsymbol{S}) \leq \boldsymbol{T}_{\varepsilon}\left(\boldsymbol{S}^{+}\left(r_{0}\right)\right) \leq \boldsymbol{S}^{+}\left(r_{0}\right)
$$

for $\boldsymbol{S} \in \widehat{\mathbb{Y}}$ and $\varepsilon \in(0,1)$. Part (b) follows from (9.24) and (9.25).

We now turn to the proof of Part (c), which requires further specification of the vector $\boldsymbol{\lambda}$. For each $i=1, \ldots, k$, let the functions $A_{i}(t), B_{i}(t):[0, \infty) \rightarrow$ $\mathbb{R}_{+}$be as defined in Theorem 8.1 and Corollary 8.2 for the particular case where $S:=\widetilde{S}_{i}, S^{ \pm}:=\widetilde{S}_{i \pm 1}, F:=F_{i}, T_{\varepsilon}:=T_{\varepsilon, i}, P_{ \pm}:=P_{i}^{ \pm}=1 \mp \delta_{i}^{ \pm}$, $\Lambda_{0}=2, \Lambda_{1}=2^{m+1}, \mu_{0}:=\left(1 / \Lambda_{1}\right)=\left(1 / 2^{m+1}\right), \mu_{1}:=\left(\Lambda_{1} / \Lambda_{0}\right)=2^{m}$, and $r_{0}=2 \ln \left(\Re_{1} / \Re_{0}\right) / \ln (2)$. For each $i=1,2, \ldots, k$, we write $A_{i}(t)=A_{0, i}>0$ and $B_{i}(t)=B_{0, i} \in(0,1 / 2)$ for $t \in[0,1]$, and $A_{i}(t)=A_{1, i} \geq A_{0, i}$ and $B_{i}(t)=B_{1, i}=1-B_{0, i} \in(1 / 2,1)$ for $t \in(1, \infty)$. For each $i=1, \ldots, k$, and for any small value $\kappa>0$, we use $\mathfrak{M}_{i}(\kappa)$ to denote the set of all ordered triples $\left(\lambda_{-}, \lambda, \lambda_{+}\right)$such that $\lambda \in\left[\Lambda_{0}, \Lambda_{1}\right]$ and $\lambda_{ \pm} \in\left[1, \Lambda_{1}\right]$, and such that the values $\mu^{ \pm}:=\left(\lambda_{ \pm} / \lambda\right) \in\left[\mu_{0}^{q_{0}}, \mu_{1}^{q_{0}}\right] \cap\left[\mu_{1}^{-q_{0}}, \mu_{0}^{-q_{0}}\right]$ satisfy (8.4) and (8.5) with $\mu_{0}:=1 / \Lambda_{1}, \mu_{1}:=\Lambda_{1} / \Lambda_{0}, q_{0}:=1 / r_{0}, C_{0}:=\lambda^{\kappa}, A(t):=A_{i}(t)$, and $B(t):=B_{i}(t)$. Let $\mathfrak{M}(\kappa)$ denote the family of all ordered $k$-tuples $\boldsymbol{\lambda}=$ $\left(\lambda_{1}, \lambda_{2}, \ldots, \lambda_{k}\right)$ such that $\left(\lambda_{i-1}, \lambda_{i}, \lambda_{i+1}\right) \in \mathfrak{M}_{i}(\kappa)$ for $i=1,2, \ldots, k$, where we set $\lambda_{0}=\lambda_{k+1}=1$.

We will first prove part (c) under the additional assumption that $\mathfrak{M}(\kappa) \neq$ $\emptyset$ for some value $\kappa>0$. Let $\boldsymbol{\lambda}=\left(\lambda_{1}, \ldots, \lambda_{k}\right)$ denote a fixed $k$-tuple in $\mathfrak{M}(\kappa)$. In view of results in $\S 2.2$, it is also clear from $(9.1 \mathrm{a}, \mathrm{b})$ that for any $i \in\{1, \ldots, k\}, r \in\left[0, r_{0}\right]$, and $\rho \in\left(0, \rho_{1}\right]$ such that $S_{i-1}^{-}(r) \in$ $\mathbb{X}^{-}\left(\Re_{0}, \Re_{1} ; \rho\right)$ (resp. $\left.S_{i+1}^{+}(r) \in \mathbb{X}^{+}\left(\Re_{0}, \Re_{1} ; \rho\right)\right)$, we have that $S_{i}^{-}(r) \in$ $\mathbb{X}^{-}\left(\Re_{0}, \Re_{1} ;\left(1 / \Lambda_{1}\right)^{r_{0}} \rho\right)$ (resp. $S_{i}^{+}(r) \in \mathbb{X}^{+}\left(\Re_{0}, \Re_{1} ;(1-h) \rho\right)$ ). It follows by induction from this (and the fact that $S_{0}^{-}(r)=\widetilde{S}_{0} \in \mathbb{X}^{-}\left(\Re_{0}, \Re_{1} ; \rho_{0}\right)$, $S_{k+1}^{+}(r)=\widetilde{S}_{k+1} \in \mathbb{X}^{+}\left(\Re_{0}, \Re_{1} ; \rho_{0}\right)$, and $\left.(1-h)^{k}>P_{*}^{+}\right)$that

$$
S_{i}^{ \pm}(r) \in \mathbb{X}^{ \pm}\left(\Re_{0}, \Re_{1} ; \rho_{2}\right)
$$

for all $i=0,1, \ldots, k, k+1$ and $r \in\left[0, r_{0}\right]$, where the constant $\rho_{2}:=$ $\min \left\{P_{*}^{+},\left(1 / \Lambda_{1}\right)^{k r_{0}}\right\} \rho_{1}>0$ was introduced previously. It is also clear from $(9.1 \mathrm{a}, \mathrm{b}),(9.3)$, and $(9.26)$ that for each $i=1, \ldots, k$ and each $r \in\left[0, r_{0}\right]$, the triples

$$
\begin{gathered}
\left(S_{-}^{-}(r), S_{-}(r), S_{-}^{+}(r)\right):=\left(S_{i-1}^{-}(r), S_{i}^{-}(r), S_{i+1}^{-}(r)\right) \in \mathbf{X}_{3}^{-}\left(\Re_{0}, \Re_{1} ; \rho_{2}\right), \\
\left(S_{+}^{-}(r), S_{+}(r), S_{+}^{+}(r)\right):=\left(S_{i-1}^{+}(r), S_{i}^{+}(r), S_{i+1}^{+}(r)\right) \in \mathbf{X}_{3}^{+}\left(\Re_{0}, \Re_{1} ; \rho_{2}\right)
\end{gathered}
$$

satisfy the requirements of Theorem 8.4, Eqs. (8.11) and (8.12), respectively, in the particular case where $\mu_{0}:=1 / \Lambda_{1}, \mu_{1}:=\Lambda_{1} / \Lambda_{0}, q_{0}:=1 / r_{0}, C_{0}:=\lambda_{i}^{\kappa}$, $S:=\widetilde{S}_{i}, S^{ \pm}:=\widetilde{S}_{i \pm 1}, F:=F_{i}, T_{\varepsilon}:=T_{\varepsilon, i}, \lambda:=\lambda_{i}, \lambda_{ \pm}:=\lambda_{i \pm 1}, P_{-}:=P_{i}^{-}$, $\widehat{P}_{-}:=P_{i+1}^{-}, P_{+}:=P_{i}^{+}, \widehat{P}_{+}:=P_{i-1}^{+}$, and $\rho_{0}:=\rho_{2}$. In view of (9.17) and 
(9.18), it follows from Theorem 8.4 that for each $i=1, \ldots, k$, there exist a value $\kappa_{0, i}>0$ and a null function $z_{i}(\cdot)$ such that

$$
\lambda_{i}^{\left(\kappa_{0, i} r-z_{i}(\varepsilon)\right) \varepsilon} S_{i}^{-}(r) \leq T_{\varepsilon, i}\left(S_{i-1}, S_{i}, S_{i+1}\right) \leq \lambda_{i}^{\left(z_{i}(\varepsilon)-\kappa_{0, i} r\right) \varepsilon} S_{i}^{+}(r)
$$

for any $r \in\left[0, r_{0}\right], \varepsilon \in(0,1)$, and ordered triple $\left(S_{i-1}, S_{i}, S_{i+1}\right) \in \mathbf{X}_{3}\left(\Re_{0}, \Re_{1}\right)$ such that

$$
S_{i}^{-}(r) \leq S_{i} \leq S_{i}^{+}(r), \quad S_{i \pm 1}^{-}(r) \leq S_{i \pm 1} \leq S_{i \pm 1}^{+}(r) .
$$

At this point, the asserted inequality (9.6) (with $\kappa_{0}=\min \left\{\kappa_{0, i}: i=\right.$ $1, \ldots, k\}$ and $z_{0}(\varepsilon)=\max \left\{z_{i}(\varepsilon): i=1, \ldots, k\right\}$ for any $\left.\varepsilon \in[0,1)\right)$ follows from (9.27), in view of Lemma 2.5(a) and the fact that $S_{0}^{-}(r) \leq \widetilde{S}_{0} \leq S_{0}^{+}(r)$ and $S_{k+1}^{-}(r) \leq \widetilde{S}_{k+1} \leq S_{k+1}^{+}(r)$ for $r \in\left[0, r_{0}\right]$ (see (9.21)).

To complete the proof of Theorem 9.4(c), we will show $\mathfrak{M}(\kappa) \neq \emptyset$ for sufficiently small $\kappa>0$. For given $\kappa>0$, we seek a $k$-tuple $\boldsymbol{\lambda}=\left(\lambda_{1}, \ldots, \lambda_{k}\right) \in$ $\mathfrak{M}(\kappa)$ in the form (7.5), where either $k=2 m$ or $k=2 m-1$ for some $m \in \mathbb{N}$. For $\boldsymbol{\lambda}=\left(\lambda_{1}, \cdots, \lambda_{k}\right)$ in this form, the condition that $\left(\lambda_{i-1}, \lambda_{i}, \lambda_{i+1}\right) \in$ $\mathfrak{M}_{i}(\kappa)$ for $i \in\{1, \ldots, k\}, i \neq m$, reduces, according to Remark 8.3(b), to the requirements that $\lambda_{i-1}^{\alpha_{i}} \lambda_{i+1}^{\beta_{i}} \leq \lambda_{i}^{\left(1-\kappa_{1, i}\right)}$ for $i=1, \ldots, m-1$, and $\lambda_{i-1}^{\beta_{i}} \lambda_{i+1}^{\alpha_{i}} \leq \lambda_{i}^{\left(1-\kappa_{1, i}\right)}$ for $i=m+1, \ldots, k$, where $\alpha_{i}=A_{0, i} B_{0, i} /\left(A_{0, i} B_{0, i}+\right.$ $\left.A_{1, i} B_{1, i}\right) \in(0,1 / 2), \beta_{i}=\left(1-\alpha_{i}\right) \in(1 / 2,1), \kappa_{1, i}=\kappa /\left(A_{0, i} B_{0, i}+A_{1, i} B_{1, i}\right)>$ 0 , all for each $i \neq m$. It is sufficient to replace these conditions by the requirements (7.7) and (7.8), where we define $\alpha:=\min \left\{\alpha_{i}: i \neq m\right\} \in(0,1 / 2)$, $\beta:=(1-\alpha)=\max \left\{\beta_{i}: i \neq m\right\} \in(1 / 2,1)$, and $\kappa_{1}=\max \left\{\kappa_{1, i}: i \neq m\right\}>0$. The condition that $\left(\lambda_{m-1}, \lambda_{m}, \lambda_{m+1}\right) \in \mathfrak{M}_{m}(\kappa)$ reduces (also according to Remark 8.3(b)) to the requirement (7.9), where $B \in\left\{B_{0, m}, B_{1, m}\right\}$ and $\kappa_{2}=\kappa / A_{0, m}>0$. It is seen by the arguments in $\S 7.12$ that the conditions (7.7), (7.8), and (7.9) are all satisfied for sufficiently small $\kappa>0$ by defining $\boldsymbol{\lambda}$ by (7.10) and (7.11).

It remains to validate our a priori assumption that $\lambda_{i} \in\left[\Lambda_{0}, \Lambda_{1}\right]:=$ $\left[2,2^{m+1}\right]$ for $i=1, \ldots, k$. Since this assumption played a role in the above theoretical determination of the values $\alpha, \beta$, and $\kappa$, it is important that its validation apply to any admissible choice of $\alpha, \beta$, and $\kappa$. Here, we call the triple $(\alpha, \beta, \kappa)$ admissible if $\alpha \in(0,1 / 2), \beta=(1-\alpha) \in(1 / 2,1)$, and $\kappa_{1}=\kappa_{1}(\kappa) \in\left(0,1-2(\alpha \beta)^{1 / 2}\right)$. For any admissible $(\alpha, \beta, \kappa)$, we have that $0<R_{1}<R_{2}<1$. Therefore, it follows from (7.10) that

$$
\begin{aligned}
\ln \left(\lambda_{m}\right) & =\left(\left(R_{2}^{m}-R_{1}^{m}\right) /\left(R_{2}-R_{1}\right)\right) \ln \left(\lambda_{1}\right) \\
& =\left(R_{1}^{m-1}+R_{1}^{m-2} R_{2}+R_{1}^{m-3} R_{2}^{2}+\cdots+R_{2}^{m-1}\right) \ln \left(\lambda_{1}\right) \leq m \ln \left(\lambda_{1}\right) .
\end{aligned}
$$

Thus, we have $\lambda_{m} \leq \lambda_{1}^{m}$. A similar argument based on (7.11) shows that $\lambda_{m} \leq \lambda_{k}^{(k+1-m)}$ for admissible $(\alpha, \beta, \kappa)$. It easily follows that $\lambda_{1}, \lambda_{k} \geq$ $\lambda_{m}^{(1 /(m+1))}$ for $k=2 m-1$ or $k=2 m$, independent of admissible $(\alpha, \beta, \kappa)$. 
Therefore, by making the arbitrary choice $\lambda_{m}=2^{m+1}$, we conclude that $\lambda_{i} \in\left[2,2^{m+1}\right]$ for $i=1, \ldots, k$, independent of admissible $(\alpha, \beta, \kappa)$.

9.10. Proof of Theorem 9.5 and Remark 9.6(a). The inequalities (9.8) and (9.9) follow from the fact that

$$
\alpha S_{i}^{-}(r) \geq S_{i}^{-}(r-\sigma) ; \quad \beta S_{i}^{+}(r) \leq S_{i}^{+}(r-\tau),
$$

both for any $r \in\left(0, r_{0}\right]$ and $i=1, \ldots, k$, where $\alpha, \beta, \sigma, \tau \geq 0$ denote values such that

$$
0<\beta \leq \lambda_{i}^{-\tau} \leq 1 \leq \lambda_{i}^{\sigma} \leq \alpha
$$

for $i=1, \ldots, k$. In fact (9.8) (resp. (9.9)) follows from (9.28a) (resp. $(9.28 \mathrm{~b}))$ in the case where $\alpha=(1 / \beta)=\left(\min \left\{\lambda_{i}\right\}\right)^{\left(\kappa_{0} r-z_{0}(\varepsilon)\right) \varepsilon}$ and $\sigma=$ $\tau=C\left(\kappa_{0} r-z_{0}(\varepsilon)\right) \varepsilon$. To prove (9.28), we first observe that (9.28a) holds trivially for $i=0$, while (9.28b) holds trivially for $i=k+1$ (where we have defined $S_{0}^{-}(r)=\widetilde{S}_{0}$ and $S_{k+1}^{+}(r)=\widetilde{S}_{k+1}$, both for any $\left.r \in\left[0, r_{0}\right]\right)$. Now if, for a given integer $i \in\{1, \ldots, k\}$, we have

$$
\alpha S_{i-1}^{-}(r) \geq S_{i-1}^{-}(r-\sigma) ; \quad \beta S_{i+1}^{+}(r) \leq S_{i+1}^{+}(r-\tau),
$$

for all $r \in\left[0, r_{0}\right]$, then it follows by (9.1) and (9.29) that

$$
\begin{aligned}
\alpha S_{i}^{-}(r) & =\max \left\{\alpha \lambda_{i}^{-r} \widetilde{S}_{i}, \alpha P_{i}^{-} S_{i-1}^{-}(r)\right\} \\
& \geq \max \left\{\lambda_{i}^{\sigma-r} \widetilde{S}_{i}, P_{i}^{-} S_{i-1}^{-}(r-\sigma)\right\}=S_{i}^{-}(r-\sigma), \\
\beta S_{i}^{+}(r) & =\min \left\{\beta \lambda_{i}^{r} \widetilde{S}_{i}, \beta P_{i}^{+} S_{i+1}^{+}(r)\right\} \\
& \leq \min \left\{\lambda_{i}^{r-\tau} \widetilde{S}_{i}, P_{i}^{+} S_{i+1}^{+}(r-\tau)\right\}=S_{i}^{+}(r-\tau) .
\end{aligned}
$$

Therefore, (9.28) holds by induction.

For the proof of Remark 9.6(a), observe that $S_{i}^{-}(r)=\lambda_{i}^{-r} \widetilde{S}_{i}$ and $S_{i}^{+}(r)=$ $\lambda_{i}^{r} \widetilde{S}_{i}$ for $i=1, \ldots, k$, provided that $r>0$ is sufficiently small. Therefore,

$$
\begin{aligned}
& \lambda_{i}^{\left(\kappa_{0} r-z_{0}(\varepsilon)\right) \varepsilon} S_{i}^{-}(r)=\lambda_{i}^{\left(\kappa_{0} r-z_{0}(\varepsilon)\right) \varepsilon-r} \widetilde{S}_{i}=S_{i}^{-}\left(r-\left(\kappa_{0} r-z_{0}(\varepsilon)\right) \varepsilon\right), \\
& \lambda_{i}^{\left(z_{0}(\varepsilon)-\kappa_{0} r\right) \varepsilon} S_{i}^{+}(r)=\lambda_{i}^{\left(z_{0}(\varepsilon)-\kappa_{0} r\right) \varepsilon+r} \widetilde{S}_{i}=S_{i}^{+}\left(r-\left(\kappa_{0} r-z_{0}(\varepsilon)\right) \varepsilon\right)
\end{aligned}
$$

both for $i=1, \ldots, k$, provided that $r>0$ and $\varepsilon>0$ are both sufficiently small.

9.11. Concluding remarks. (a) The author believes that by relatively straight-forward modifications, the present convergence proof will extend to several modified versions of Problem 1.1. First, one can generalize (1.2) such that for each $i=1, \ldots, k+1$, the function $U_{i}(x)$ is $p_{i}$-harmonic (for a given constant $p_{i}>1$ ) in the annular domain $\Omega_{i}$ (see $[6,10,17]$ ). Secondly, one can let the outermost boundary $S_{k+1}$ become a free boundary characterized by the requirement that $\left|\nabla U_{k+1}(x)\right|=A(x)$ on $S_{k+1}$, where the given positive function $A(x)$ has suitable properties (guaranteeing the starlikeness of solutions relative to some ball). Thirdly, one can consider the (essentially) 
limiting case of Problem 1.1 (or either of the above modified versions) in which the layers are horizontally infinite, and the layer boundaries are the graphs of functions $z=S_{i}(y): \mathbb{R}^{N-1} \rightarrow \mathbb{R}$, each of which becomes constant in the limit as $|y| \rightarrow \infty$.

(b) At the cost of abandoning a general mathematical convergence analysis, the above "operator method" can be modified to produce various related trial free-boundary operators (for Problem 1.1) with improved convergence properties, as observed in test cases such as the limiting case of infinite parallel planes. For example, one can seek to accelerate convergence by multiplying the forward progression induced by the operator by a factor $\lambda>1$ at each step of the iteration. Given a smooth iterate $\boldsymbol{S}_{n}=\left(S_{n, 1}, \ldots, S_{n, k}\right)$, one defines $\boldsymbol{S}_{n+1}$ such that $S_{n+1, i}=\left\{x+\lambda \phi_{n, i}\left(x, \varepsilon_{n}\right) \nu_{n, i}(x): x \in S_{n, i}\right\}$ for $i=1, \ldots, k$, where $\nu_{n, i}(x)$ denotes the exterior unit normal to $S_{n, i}$ at $x \in S_{n, i}$, and $\phi_{n, i}(x, \varepsilon)$ denotes the translation of $S_{n, i}$ in the direction $\nu_{n, i}(x)$ which is induced by applying the operator $\boldsymbol{T}_{\varepsilon_{n}}$ to $\boldsymbol{S}_{n}$. As a limiting case (as $\varepsilon_{n} \downarrow 0$, where $\lambda=\left(\mu / \varepsilon_{n}\right)$ for some fixed $\left.\mu \in(0,1]\right)$, one defines $\boldsymbol{S}_{n+1}$ such that $S_{n+1, i}=\left\{x+\mu \phi_{n, i}(x) \nu_{n, i}(x): x \in S_{n, i}\right\}$ for $i=1, \ldots, k$, where $\phi_{n, i}(x)=\operatorname{limit}_{\varepsilon \rightarrow 0+}\left(\phi_{n, i}(x, \varepsilon) / \varepsilon\right)$ for $x \in S_{n, i}$. The functions $\phi_{n, i}(x): S_{n, i} \rightarrow \mathbb{R}, i=1, \ldots, k$, solve the equations

$$
F_{i}\left(x,\left[\left|\nabla U_{n, i}\right| /\left(1+\phi_{n, i}\left|\nabla U_{n, i}\right|\right)\right],\left[\left|\nabla U_{n, i+1}\right| /\left(1-\phi_{n, i}\left|\nabla U_{n, i+1}\right|\right)\right]\right)=0,
$$

where the functions $U_{n, 1}, \ldots, U_{n, k+1}$ solve (1.2) in the case where $\boldsymbol{S}:=\boldsymbol{S}_{n}$, and the derivatives exist.

\section{References}

[1] A. Acker, Free-boundary optimization - a constructive, iterative method, J. Appl. Math. Phys., (ZAMP), 30 (1979), 885-900.

[2] How to approximate the solutions of certain free boundary problems for the Laplace equation by using the contraction principle, J. Appl. Math. Phys., (ZAMP), 32 (1981), 22-33.

[3] - On the convexity and successive approximation of solutions in a free boundary problem with two fluid phases, Comm. in Part. Diff. Eqs., 14 (1989), 1635-1652.

[4] - On the multilayer problem: Regularity, uniqueness, convexity, and successive approximation of solutions, Comm. in Part. Diff. Eqs., 16 (1991), 647-666.

, On the existence of convex classical solutions to multilayer fluid problems in arbitrary space dimensions, Pacific J. of Math., 162 (1994), 201-231.

[6] _ On 2-layer free boundary problems with generalized joining conditions: Convexity and successive approximation of solutions, in 'Comparison Methods and Stability Theory' (X. Liu and D. Siegel, editors), Lecture notes in Pure and Applied Mathematics, Vol. 162, New York, Marcel Dekker, Inc., 1994.

Operator methods for free-surface and free-interface problems in fluid dynam$i c s$, Proceedings of the Conference on Differential Equations and Dynamical Systems, University of Waterloo, Ontario, Aug. 1-4, 1997, Edited by D. Siegel and X. Liu. 
[8] - On the existence of convex classical solutions to multilayer free boundary problems with general nonlinear joining conditions, Trans. Amer. Math. Soc., 350 (1998), 2981-3020.

[9] A. Acker, E. Kadakal and K. Miller, A trial free boundary method for computing Batchelor flows, J. Compu. Appl. Math., 80 (1997), 31-48.

[10] A. Acker and R. Meyer, A free boundary problem for the p-Laplacian: Uniqueness, convexity, and successive approximation of solutions, Electronic J. Diff. Eqs., 1995(8) (1995), 1-20.

[11] H.W. Alt, L.A. Caffarelli and A. Friedman, Variational problems with two fluid phases and their free boundaries, Trans. Amer. Math. Soc., 282 (1984), 431-461.

[12] J. Crank, Free and Moving Boundary Problems, Clarendon Press, Oxford, 1984.

[13] C.W. Cryer, Numerical methods for free and moving boundary problems, Doc. Nr. 2/86 N., Inst. für numerische und instrumentelle Mathematik, Westfälische WilhelmsUniversität zu Münster, Einsteinstr. 62, D-4000 Münster, Germany.

[14] D. Gilbarg and N.S. Trudinger, Elliptic Partial Differential Equations of Second Order, (2nd edition), Springer-Verlag, 1983.

[15] E. Kadakal, On the successive approximation of solutions to some elliptic free boundary problems, Doctoral Dissertation, Department of Mathematics and Statistics, Wichita State University, June, 1996.

[16] P. Laurence and E. Stredulinsky, Existence of regular solutions with convex level sets for semilinear elliptic equations with non-monotone $L^{1}$-nonlinearities, Part I: An approximating free boundary problem, Indiana U. Math. J., 39 (1990), 1081-1114.

[17] R. Meyer, Approximation of the solutions of free boundary problems for the p-Laplace equation, Doctoral Dissertation, Department of Mathematics and Statistics, Wichita State University, May, 1993.

Received April 21, 1997 and revised January 5, 1998.

Wichita State University

WICHITA, KS 67260-0033

E-mail address: acker@twsuvm.uc.twsu.edu 